\title{
SUPPLEMENTS TO THE ATLAS OF BUTTERFLIES (LEPIDOPTERA: RHOPALOCERA) OF SLOVENIA
}

\author{
Tatjana ČELIK ${ }^{1}$
}

\begin{abstract}
The Atlas of butterflies (Lepidoptera: Rhopalocera) of Slovenia (Verovnik et al. 2012) treats 179 butterfly species whose occurrence in Slovenia is confirmed, and 24 species for which only unconfirmed data exist. By the end of 2011, 212.936 distribution records by 167 contributing authors were collected for the Atlas. The Digital database of butterflies of the Jovan Hadži Institute of Biology of the Scientific Research Centre of the Slovenian Academy of Sciences and Arts was not considered in the process of collecting the data for the Atlas. This database contains 30.630 distribution records for butterfly species found in Slovenia from research projects undertaken by the Institute from 1995 to date. Given that this rich data source was not included in the Atlas, I here provide a supplement to the existing Atlas, reporting 1529 new records for 140 butterfly species. These updates provide new areas or range limits for 14 species, the presence of 71 species in areas the Atlas designated as "white spots", new information on 3 species with very limited distribution in Slovenia, and additional corroboration for distribution of 27 species that the Atlas covered.
\end{abstract}

Key words: butterflies, distribution, Slovenia, Atlas.

\section{Izvleček}

Atlas dnevnih metuljev (Lepidoptera: Rhopalocera) Slovenije (Verovnik et al. 2012) obravnava 179 vrst, katerih prisotnost v Sloveniji je potrjena, in 24 vrst, za katere obstajajo le nepotrjene navedbe o najdbah na ozemlju Slovenije. Do konca leta 2011 je bilo za Atlas zbranih 212.936 podatkov, ki jih je prispevalo 167 avtorjev. Pri zbiranju podatkov za Atlas ni bila upoštevana Digitalna podatkovna zbirka dnevnih metuljev Biološkega inštituta Jovana Hadžija Znanstvenoraziskovalnega centra Slovenske akademije znanosti in umetnosti. Zbirka obsega 30.630 podatkov o vrstah dnevnih metuljev najdenih na ozemlju Slovenije, pridobljenih večinoma pri raziskovalnih projektih Biološkega inštituta J. H. ZRC SAZU, ki so potekali od leta 1995 naprej. S primerjavo med podatki v Atlasu in v inštitutski podatkovni zbirki sem ugotovila, da je smiselno objaviti dopolnitve $\mathrm{k}$ obstoječemu Atlasu. Dopolnitve obsegajo 1529 podatkov za 140 vrst. Med njimi je 14 vrst za katere so opredeljena nova območja in meje razširjenosti v Sloveniji, in 71 vrst najdenih v območjih, ki so v Atlasu opredeljena kot "bele lise". Dopolnitve vključujejo tudi nove informacije o nahajališčih 3 vrst z zelo omejeno razširjenostjo v Sloveniji in o pojavljanju 27 vrst na že znanih nahajališčih.

Ključne besede: dnevni metulji, razširjenost, Slovenija, Atlas.

\section{INTRODUCTION}

Atlas of butterflies (Lepidoptera: Rhopalocera) of Slovenia (Verovnik et al. 2012, 456 p.; hereafter: Atlas) was published in spring 2012 and includes seven basic chapters with the central, most extensive thematic chapter, i.e. Species overview (pp. 73-444) treating 179 species which reliably occur in Slovenia, and 24 species for which there are only unconfirmed data of their presence in Slovenia. Among 179 confirmed species, there are 4 species considered extinct: Pyrgus onopordi (Rambur, 1839) (in Slovenia last seen 100 years ago), Nymphalis xanthomelas (Esper, 1781) (in Slovenia last seen more than 40 years ago), $\mathcal{N}$. vaualbum (Denis \& Schiffermüller, 1775) (in Slovenia

\footnotetext{
${ }^{1}$ Jovan Hadži Institute of Biology (BIJH), Scientific Research Centre of the Slovenian Academy of Science and Arts (ZRC SAZU), Novi trg 2, P. O. Box 306, SI-1001 Ljubljana, Slovenia, e-mail: tcelik@zrc-sazu.si
} 
last seen over 70 years ago) and Hyponephele lycaon (Kühn, 1774) (in Slovenia last seen 30 years ago). Presentation of each of the 179 species (pp. 73-435) includes a graphical part (species distribution, phenology, vertical distribution and habitats in Slovenia) and text (comments to the species distribution in Slovenia; short description of species' biology and threatened status summarized above all upon references listed on pages 442-444). The species distribution is shown on two maps: (a) a map with localities which minimal spatial accuracy is defined by the size of the area of $100 \mathrm{~km}^{2}$, and (b) a map with the presence of species in a $5 \times 5 \mathrm{~km}$ UTM square. On both maps the species' distribution records are provided for two time periods: locality $/ 5 \times 5 \mathrm{~km}$ UTM square where the species was found before year 1990 , and the locality $/ 5 \times 5 \mathrm{~km}$ UTM square with the presence of species after year 1989 .

For 203 species (i.e. 179+24) discussed in the Atlas, $96.1 \%$ of $5 \times 5 \mathrm{~km}$ UTM squares covering the territory of Slovenia were investigated, and 212.936 distribution records (museum and private collections: 15.864, published references: 33.571, field surveys: 163.501) were collected by the end of 2011 (Verovnik et al. 2012: pp. 40). 167 authors contributed the records to the Atlas (Verovnik et al. 2012: pp. 8) but this excludes the authors whose unpublished data (from studies, reports, etc.) were also used for the Atlas (cf. Verovnik et al. 2012: pp. 30). These authors are only mentioned in the chapter Sources (cf. Verovnik et al. 2012: pp. 445-453).

Digital database of butterflies of Jovan Hadži Institute of Biology of the Scientific Research Centre of the Slovenian Academy of Sciences and Arts (hereafter: BIJH ZRC SAZU) was not considered in the process of collecting the data for the Atlas. This database covers data collected within two major national lepidopterological projects conducted by the BIJH ZRC SAZU (both projects involve members of the Slovenian Entomological Society Štefan Michieli, Centre for Cartography of Fauna and Flora, Department of Biology of Biotechnical Faculty of University of Ljubljana, non-resident co-workers): the "Atlas of threatened butterflies of Slovenia" (Čelik \& Rebeušek 1996) and the "Expertise on fundaments for establishment of Natura 2000 network in Slovenia - Lepidoptera" (Čelik et al. 2004). The major part of this database includes data collected by the BIJH ZRC SAZU Research Program and other research projects which took place from 1995 onwards. The Digital database of butterflies of BIJH ZRG SAZU contains 30.630 distribution records for species found in Slovenia (collection: 15\%, published references: 13\%, field surveys: $72 \%$ ), among which $86 \%$ are records on the presence of species after year 1989. Not taking into account the records obtained by both national lepidopterological projects mentioned above, the 23.508 distribution records (collection: $1 \%$, published references: $9 \%$, field surveys: $90 \%$ ) for butterfly species present in Slovenia are included in the database, of which $99 \%$ are records on the presence of species after year 1989.

By comparing the data presented in the Atlas in the main chapter "Species overview", and the data from Digital database of the BIJH ZRC SA$\mathrm{ZU}$, I found out that regarding the type and age of the data in our database (field surveys: $90 \%$ of the records, $99 \%$ records after 1989), it is reasonable to publish this supplement to the existing Atlas. My updates thus contribute to a more comprehensive and detailed knowledge of the distribution of butterfly species in Slovenia, which is the basis for a complex and effective planning of further studies on ecology, population and genetic diversity of species, on biodiversity of Slovenia, and for objective and argumentative spatial planning for nature conservation.

\section{METHODS}

For a comparison between the information presented in the Atlas in the main chapter "Species overview" (pp. 78-435) and the data from Digital database of butterflies of the BIJH ZRC SAZU, only my data (published and unpublished) from the database were used, consisting of 19.841 records (collection: 0.6\%, field surveys: 99.4\%; records before year 1990: 0.6\%, after year 1989: 99.4\%). In the Atlas, many of my previously published data including the "grey literature" like studies, reports, etc., were used (see Chapter "Sources" in Verovnik et al. 2012: pp. 445-453). Each distribution record on the distribution maps in the Atlas provides only information about the record date, either before 1990 or after 1989 , and spatial accuracy only at the level of $5 \times 5 \mathrm{~km}$ UTM square (see Introduction). Hence, I could use here only those of my unpublished records that represent novel time/spatial information on the accuracy levels used in the Atlas (see below). Considering the type and age of my records, only species which are confirmed for Slovenia (179 
species) were included in the preparation of this supplement to the Atlas.

I here provide: (1) supplements to the distribution map of the species shown in Atlas with $5 \times 5 \mathrm{~km}$ UTM squares, and (2) supplements to the (accompanying) text dealing with the comments to the distribution map and vertical distribution of the species. I did not add the supplements to the description of species' biology and threatened status as presented in the Atlas because they represent unpublished results of our studies which will be published elsewhere.

(1) Supplements to the distribution map of the species are classified into three groups:

(I) New data for $10 \times 10 \mathrm{~km}$ UTM square meaning that the Atlas lacks a record for this UTM square. In the chapter Results and Discussion a symbol (I) is used for records of this group.

(II) New data for $5 \times 5 \mathrm{~km}$ UTM square meaning that the Atlas lacks a record for this UTM square. In the chapter Results and Discussion a symbol (II) is used for records of this group.

(III) The records after year 1989 for $5 \times 5 \mathrm{~km}$ UTM square meaning that the Atlas provides only records preceding 1990 for this UTM square. In the chapter Results and Discussion a symbol (III) is used for records of this group.

Each record includes the following information: UTM square (letter code of $10 \times 10 \mathrm{~km}$ UTM square/number code of $5 \times 5 \mathrm{~km}$ UTM square), date, description of locality (region name, the name of a populated place or geographical features, e.g., mountain, hill, stream), altitude (in meters a. s. l.) and geographical coordinates (east/north). For the description of locality, the official geographical names in Slovene were used.

An example of the record: UL99/3: 16.5.2006, Primorska, Banjšice, Madoni, 705 m, 399411/98887.

UTM squares are designated at UTM (Universal Transverse Mercator) projection on WGS 84 ellipsoid, in UTM zone 33T. Geographical coordinates are expressed in meters and determined according to the Slovenian national coordinate system D 48 on Bessel ellipsoid (5. zone), Gauss-Krüger projection.

In the chapter Results and Discussion the records within each of three groups are given according to UTM squares. Records belonging to the same UTM square are separated by semicolons.
In the case that species was observed within the same day in a number of locations (i.e. geographical coordinates) within the same locality, the description of the locality is mentioned only once (at the first location); other locations include only information on altitude and geographical coordinates. In the case that species was observed on the same locality at different days, all dates are listed together after the information on the UTM square, and description of locality, altitude and geographical coordinates follow.

(2) Supplements to the text in Atlas dealing with the comments to the distribution map and vertical distribution of the species follow the supplements to the distribution map of the species. They are added for species for which data from Digital database of butterflies of the BIJH ZRC SAZU (author of records: T. Čelik) showed the following:

(i) the existence of new areas/range limits of the species distribution in Slovenia in comparison to the distribution presented in the Atlas;

(ii) the presence of species in areas which in the Atlas are designated as "white spots" either due to lack of records in those parts of Slovenia, or the species was not found in those areas during field surveys (cf. Verovnik et al. 2012: pp. 44-45, 73-435);

(iii) new information on species with very limited distribution in Slovenia (i.e. species range comprises less than $1 \%$ of the all $5 \times 5 \mathrm{~km}$ UTM squares in Slovenia since 1989 considering the distribution presented in Atlas);

(iv) new information on already known localities of the species (e.g., new data on species vertical distribution; new data on presence of the species in the last 10 years in areas where species was found only before 1990 according to the Atlas; new data on the abundance of adults considering the data presented in Atlas, etc.).

In the chapter Results and Discussion symbols (i), (ii), (iii) and (iv) are used for these types of supplements.

The nomenclature for butterfly species follows Van Sway et al. (2010). The species Leptidea sinapis (Linnaeus, 1758) and L. reali (Reissinger 1989) which require a preparation of genitalia for reliable identification are treated at the level of the species complex. The names of Slovenian regions mentioned in supplements of type (2) follow Perko and Kladnik (1998). 


\section{RESULTS AND DISCUSSION}

Supplements of type (1) and (2) comprise 1.529 distribution records for 140 butterfly species. Among them only 15 records (for 8 species) are from year 2012. Among 140 species, there are three species (Plebejus orbitulus (de Prunner, 1798), Euphydryas intermedia (Ménétriés, 1859), Coenonympha tullia (Müller, 1764)) for which only type (2) of supplements are added.

(1) Supplements to the distribution maps presented in Atlas of butterflies (Lepidoptera: Rhopalocera) of Slovenia (Verovnik et al. 2012) with UTM grid of $5 \times 5 \mathrm{~km}$ squares comprise:

- 137 species (no. of species: (I) $=97,($ II $)=121$, (III) = 77);

- 1518 records (no. of records: (I) $=317$, (II) $=993$, $($ III $)=208)$. Among them there are 11 records (no. of records: (I) $=3$, (II) $=7$, (III) $=1$ ) from year 2012 which include 7 species: Heteropterus morpheus (Pallas, 1771), Thymelicus sylvestris (Poda, 1761), Ochlodes sylvanus (Esper, 1777), Pieris mannii (Mayer, 1851), Pontia edusa (Fabricius, 1777), Lycaena dispar (Haworth, 1802) and Minois dryas (Scopoli, 1763);

- 73 different $10 \times 10 \mathrm{~km}$ UTM squares (suppl. of group (I)), 160 different $5 \times 5 \mathrm{~km}$ UTM squares (suppl. of group (II)) and 47 different $5 \times 5 \mathrm{~km}$ UTM squares with the records after year 1989 for which in Atlas only records before year 1990 exist (suppl. of group (III));

- 17 (1.1\%) records from the period before 1990, 280 (18.3\%) records from the period 1990 to 2000 , and 1221 (or $80.6 \%$ ) records from the period 2001-2012, i.e. 1501 (98.9\%) records after year 1989 .

(2) Supplements to the text in Atlas dealing with the comments to the distribution map and vertical distribution of the species comprise 96 species (no. of species: (i) $=14$, (ii) $=71$, (iii) $=3$, (iv) $=27$ ). Only 4 records are from year 2012, all for species $C$. tullia.

SupPlements of type (1) AND (2) TO THE AtLAS OF BUTTERFLIES (LEPIDOPTERA: Rhopalocera) of Slovenia

\section{Erynnis tages (Linnaeus, 1758)}

(I) - UM72/3: 4.6.1998, Primorska, Breginj, Prekopa, 876 m, 378245/126830; VL57/4: 16.5. 1994, Notranjska, Bloška planota, Velike Bloke, 725 m, 459808/71986; WL08/1: 26.5.2004, Dolenj- ska, Mirna, Migolska gora, 260 m, 503166/90226.

(II) - UL99/3: 16.5.2006, Primorska, Banjšice, Madoni, 705 m, 399411/98887; 18.5.2006, Primorska, Banjšice, Sveto, $701 \mathrm{~m}, 398862 / 99420$; UM91/3: 14.6.2006, Primorska, Kolovrat, Foni, 569 m, 396901/117862; VL04/2: 28.4.1994, Primorska, Koper, Škocjanski zatok, 0 m, 403178/45684; VL47/1: 11.5.1993, Notranjska, Planinsko polje, Jakovski grič, 464 m, 442830/79620.

(III) - VL15/1: 11.5.2008, Primorska, Sežana, Lipica, 371 m, 412965/58830; 369 m, 412960/ 59140; 379 m, 412840/59320; WL27/2: 18.5.2004, Dolenjska, Novo mesto, Velike Brusnice, $300 \mathrm{~m}$, $521990 / 74163$.

\section{Carcharodus alceae (Esper, 1780)}

(II) - WL14/1: 19.5.2004, Bela Krajina, Črnomelj, Talčji vrh, 240 m, 512555/49750.

(III) - VL16/2: 13.4.1995, Primorska, Sežana, Dol Leskovec, 305 m, 412246/62030.

\section{Carcharodus flocciferus (Zeller, 1847)}

(II) - UL97/3: 10.6.2005, Primorska, Kras, Novelo, 327 m, 396142/79045.

\section{Spialia sertorius (Hoffmannsegg, 1804)}

(I) - VL56/1: 7.5.1994, Notranjska, Cerkniško polje, Otoški grič, $567 \mathrm{~m}, 451875 / 66430$.

(II) - VL14/3: 11.8.2004, Primorska, Podgorski kras, Prešnica, 480 m, 416670/47890; VL16/3: 13.5.2005, 24.6.2005, Primorska, Kras, Griže (Sežana), $360 \mathrm{~m}, 418626 / 68350$.

\section{Pyrgus andromedae (Wallengren, 1853)}

(I) - VM13/3: 17.7.2009, Julijske Alpe, Pokljuka, Klečica, 1820 m, 419110/139523; VM62/1: 15.7.2005, Kamniško-Savinjske Alpe, Veliki Zvoh, 1953 m, 465167/129628; VM73/4: 21.7.2009, Kamniško-Savinjske Alpe, Dleskovška planota, 1850 m, 476127/134010; 1880 m, 475603/134083; $1835 \mathrm{~m}, 475535 / 134610 ; 1795 \mathrm{~m}, 476057 / 134883$; $1670 \mathrm{~m}, 476345 / 134925 ; 1715 \mathrm{~m}, 476193 / 134967$; $1590 \mathrm{~m}, 476702 / 135032$.

(II) - VM12/2: 25.7.2005, Julijske Alpe, Rodica, Pl. Suha, 1388 m, 412041/122509; VM63/2: 16.7.1995, Kamniško-Savinjske Alpe, Grintovec, Ovnov čer, $2005 \mathrm{~m}, 464860 / 133901 ; 16.7 .2005$, Kamniško-Savinjske Alpe, Grintovec, Spodnji Dolci, 1805 m, 464062/134294.

(i) - New areas of the species distribution: eastern part of Julian Alps, southern and eastern parts of Kamniško-Savinjske Alps (Fig. 1). 


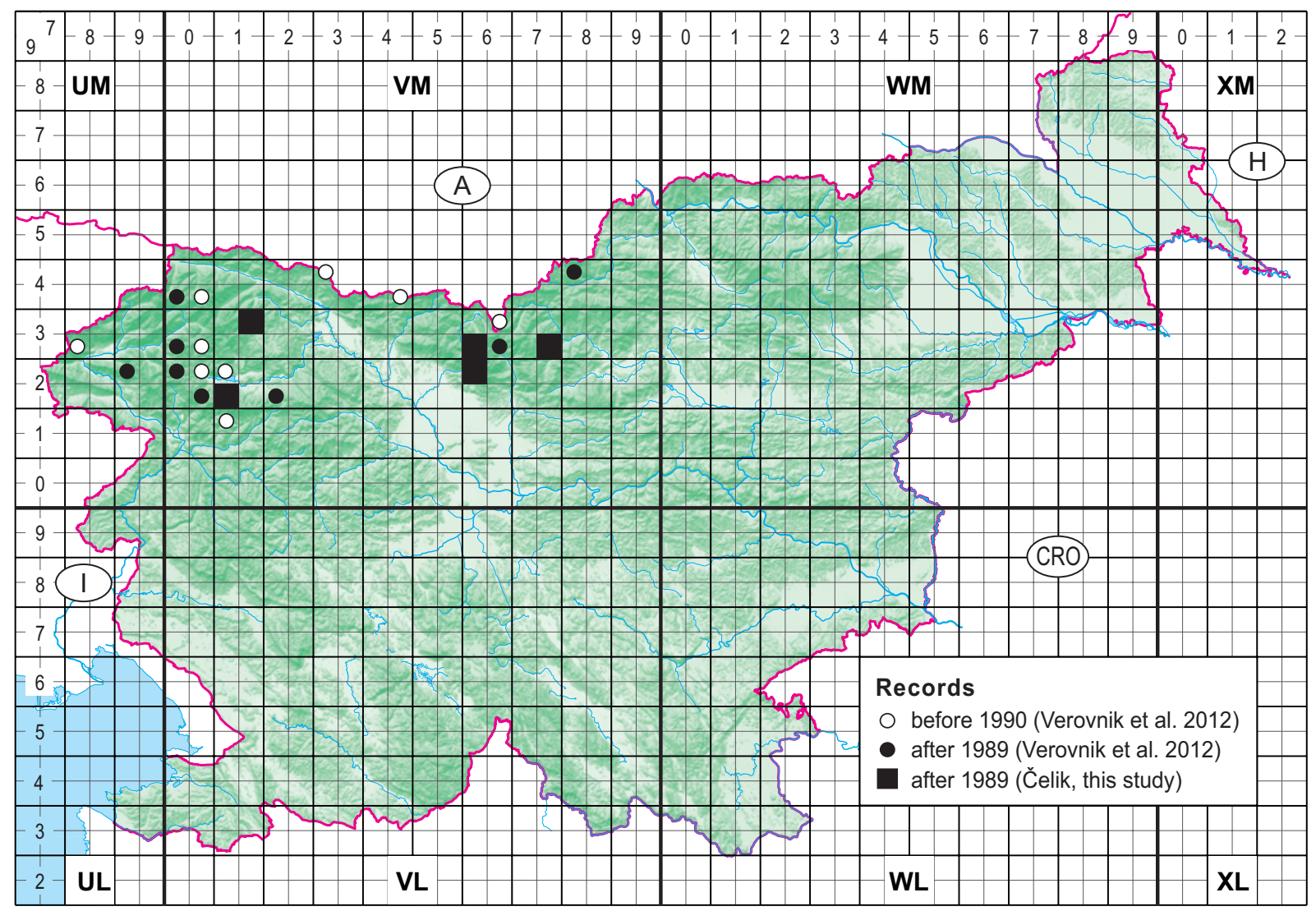

Figure 1: New records on the distribution of Pyrgus andromedae (Wallengren, 1853) (Čelik, this study) according to the Atlas of butterflies (Lepidoptera: Rhopalocera) of Slovenia (Verovnik et al. 2012: pp. 91).

Slika 1: Novi podatki o razširjenosti vrste Pyrgus andromedae (Wallengren, 1853) (Čelik, ta študija) glede na podatke v Atlasu dnevnih metuljev (Lepidoptera: Rhopalocera) Slovenije (Verovnik s sod. 2012: str. 91).

\section{Pyrgus malvae (Linnaeus, 1758)}

(I) - VL56/1: 23.6.1994, Notranjska, Cerkniško polje, Lipsenj, 551m, 454542/66056; 25.5.1994, Notranjska, Cerkniško polje, Otoški grič, 570 m, 451757/66286; 7.5.1994, Notranjska, Cerkniško polje, Otoški grič, 567 m, 451875/66430; VL56/4: 24.5.1994, Notranjska, Loška dolina, Dane, 568 m, 457111/62552; VL94/3: 20.5.2004, Dolenjska, Mozelj, Kočarji, 520 m, 497227/48495; 520 m, 497479/48832; WL04/2: 20.5.2004, Dolenjska, Knežja Lipa, Videm, 680 m, 502909/44999; WL08/1: 26.5.2004, Dolenjska, Mirna, Migolska gora, 260 m, 503166/90226; WL26/1: 28.6.1994, Dolenjska, Gorjanci, Sv. Miklavž, 941 m, 525182/ 69956; WL58/2: 28.7.2008, Dolenjska, Čateške Toplice, $297 \mathrm{~m}$, 550201/81857.

(II) - UL99/3: 16.5.2006, Primorska, Banjšice, Madoni, 755 m, 399727/98814; 732 m, 399536/ 98845; Kuk, 760 m, 400311/99855; 18.5.2006, Primorska, Banjšice, Ježevec, 731 m, 398638/99255; 708 m, 398757/99308; 701 m, 398862 99420; Sleme,
773 m, 399549/100511; VL09/1: 16.5.2006, Primorska, Banjšice, Kuk, 748 m, 400350/99650; VL47/1: 4.5.1994, Notranjska, Planinsko polje, Trzne, 447 m, 443670/78189; Podgorčev studenec, 445 m, 440919/79968; 18.5.1993, Notranjska, Planinsko polje, Gmajna, 446 m, 443006/78405; 11.5.1993, Notranjska, Planinsko polje, Jakovski grič, 464 m, 442830/79620; VL66/2: 24.5.1994, Notranjska, Loška dolina, Veliki Obrh, $582 \mathrm{~m}$, 462187/61865; VL67/3: 15.6.2004, Dolenjska, Rašica, Rob, 500 m, 467358/78088; VL69/3: 3.6.1996, Gorenjska, Ljubljana, Zg. Kašelj, 275 m, 469557/ 100312; VL99/1: 8.6.2004, Dolenjska, Veliki Gaber, Razbore, 520 m, 493100/96085; VM40/4: 23.6.2004, Gorenjska, Polhograjsko hribovje, Mačkov graben, 460 m, 447560/104250; VM61/3: 10.6.2004, Gorenjska, Tunjice, Tunjščica, $390 \mathrm{~m}$, 468353/120949; Knežji potok, 360 m, 467339/ 121034; WL17/1: 11.5.2004, Dolenjska, Ždinja vas, Sela pri Śtravberku, 540 m, 514646/79674; 18.5.2004, Dolenjska, Ždinja vas, Nova Gora, 
580 m, 514276/80220; W M86/4: 4.5.1995, Štajerska, Vučja vas, $185 \mathrm{~m}, 585857 / 162473$.

(III) - VL16/2: 13.4.1995, Primorska, Sežana, Dol Leskovec, 305 m, 412246/62030; 9.6.1995, Primorska, Sežana, Dol Leskovec, 302 m, 412228/ 62044.

\section{Pyrgus armoricanus (Oberthür, 1910)}

(I) - UL97/1: 10.6.2005, Primorska, Kras, Lokvica, 200 m, 390945/81035; UL97/3: 26.9.2005, Primorska, Kras, Novelo, 327 m, 396142/79045; VL37/3: 12.8.1993, Notranjska, Planinsko polje, Smukovci, 444 m, 440142/5080447; VL47/1: 4.8.1993, Notranjska, Planinsko polje, Jakovski grič, $449 \mathrm{~m}, 441709 / 5080462$; Čelo, $450 \mathrm{~m}$, 441230/5080703; 12.8.1993, Notranjska, Planinsko polje, Jakovski grič, 464 m, 442783/5079614; Kosovnica, $445 \mathrm{~m}, 441028 / 5080100$; 20.8.1993, Notranjska, Planinsko polje, Luže, $445 \mathrm{~m}, 443687 /$ 5078726; 6.9.1993, Notranjska, Planinsko polje, Čelo, 450 m, 441230/5080703. (i) - New areas of the species distribution: Kras, Notranjsko podolje.

\section{Pyrgus alveus (Hübner, 1803)}

(I) - VL69/4: 26.6.1995, Dolenjska, Ljubljansko barje, Škofljica, 290 m, 466077/92168; VM54/ 4: 20.7.2006, Zahodne Karavanke, Mala Košuta, 1730 m, 456910/144480; VM73/4: 21.7.2009, Kamniško-Savinjske Alpe, Dleskovška planota, $1795 \mathrm{~m}, 476057 / 134883$.

(II) - UM82/1: 6.8.2004, Primorska, Breginjski kot, Kobariški Stol, 1550 m, 382017/126977; VL59/2: 13.6.1995, Notranjska, Ljubljansko barje, Bevke, 286 m, 451729/93527.

(III) - UM83/3: 18.7.2006, Julijske Alpe, Bovec, Črnelski Vršiči, 2017 m, 386750/137840; 2157 m, 386303/137855; 2320 m, 386162/137990.

(i) - New areas of the species distribution: Kamniško-Savinjske Alps (Fig. 2). Note: According to the data in the Atlas (Verovnik et al. 2012:

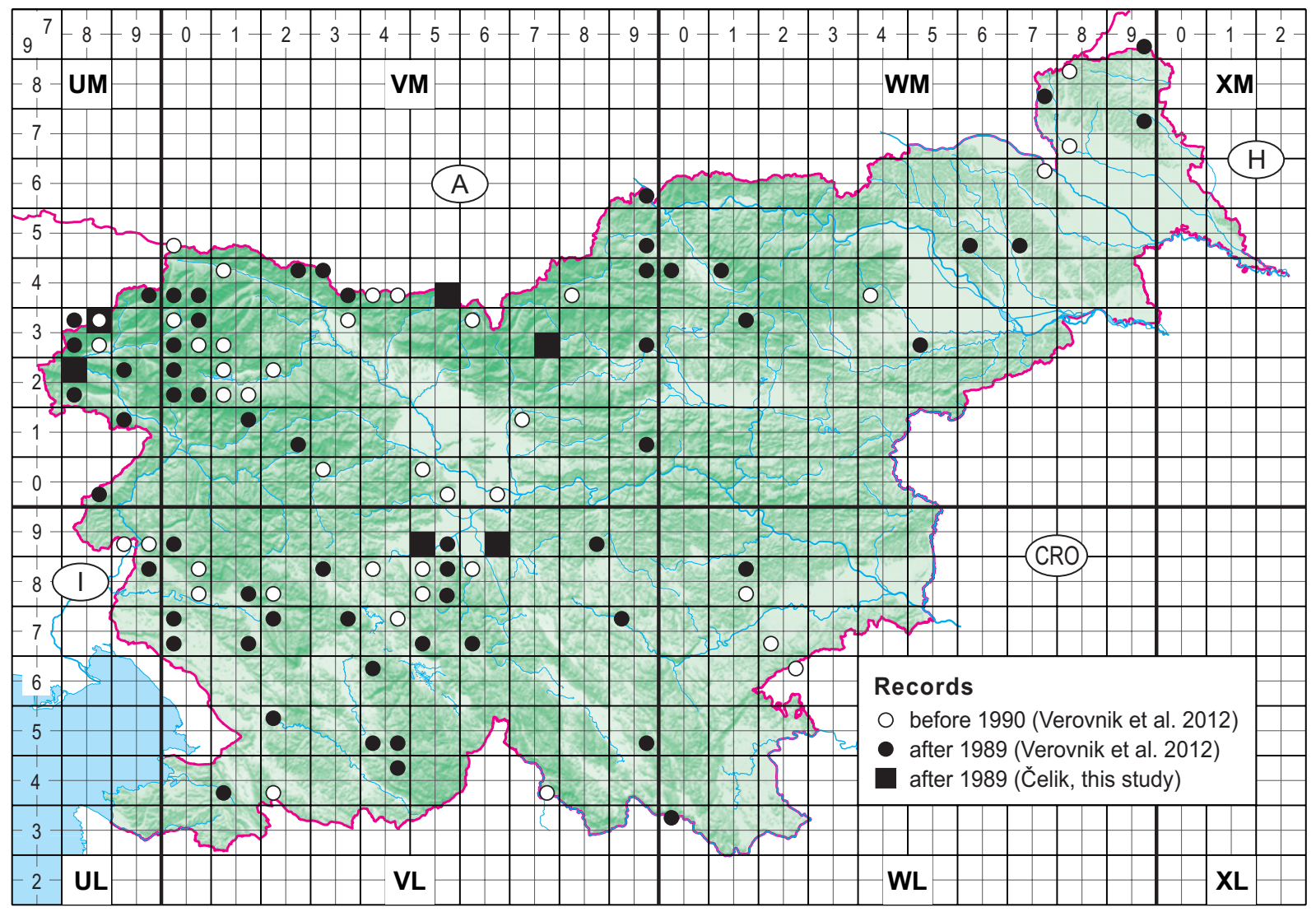

Figure 2: New records on the distribution of Pyrgus alveus (Hübner, 1803) (Čelik, this study) according to the Atlas of butterflies (Lepidoptera: Rhopalocera) of Slovenia (Verovnik et al. 2012: pp. 103).

Slika 2: Novi podatki o razširjenosti vrste Pyrgus alveus (Hübner, 1803) (Čelik, ta študija) glede na podatke v Atlasu dnevnih metuljev (Lepidoptera: Rhopalocera) Slovenije (Verovnik s sod. 2012: str. 103). 
pp. 102), in the Slovenian Alps the species occurs only in the Julian Alps and the Western Karavanke Mountains.

(iv) - New information on species occurrence in Western Karavanke Mountains: species distribution map in the Atlas (Verovnik et al. 2012: pp. 103) indicates that in eastern part of the Western Karavanke Mountains there are only three localities (three $5 \times 5$ UTM squares), all with records before 1990. My record from the mountain chain of Košuta (VM54/4) shows that in eastern part of the Western Karavanke Mountains species was also found in the last decade (Fig. 2).

\section{Heteropterus morpheus (Pallas, 1771)}

(I) - VL23/2: 21.6.2002, Primorska, Rakitovec, Ubred, $660 \mathrm{~m}, 421031 / 36003$; VL26/3: 25.6.1997, Primorska, Senožeče, Laže, 698 m, 428111/66702.

(II) - UM90/1: 30.6.2006, Primorska, Kanalski Kolovrat, Lig, 489 m, 393760/106520; 607 m, 392810/107048; UM90/4: 30.6.2006, Primorska, Banjšice, Breščaki, $632 \mathrm{~m}, 398146 / 101314$; Primorska, Kanalski Vrh, 571 m, 397110/104200; VL06/3: 5.7.2002, Primorska, Kras, Dutovlje, 275 m, 408528/69110; VL15/1: 25.6.1997, Primorska, Kras, Lokev, 400 m, 414734/59704; VL15/3: 25.6.1997, Primorska, Kras, Lokavsko polje, 431 m, 415414/57744; VL16/1: 11.6.2003, Primorska, Kras, Tomaj, 340 m, 411137/68931; VL16/2: 25.6.1997, Primorska, Sežana, Dol Leskovec, 300 m, 412212/62044; VL16/4: 11.6.2003, Primorska, Kras, Brestovica pri Povirju, 405 m, 418675/63611; 420 m, 419143/63698; 400 m, 418317/63885; VL28/ 4: 26.6.2002, Primorska, Trnovski gozd, Križna gora, 779 m, 425875/81106; VL68/4: 14.7.2012, Dolenjska, Visoko, 613 m, 467930/82100; VM11/4: 17.7.2006, Primorska, Baška grapa, Obloke, 455 m, 416278/115122; 526 m, 416241/115388.

\section{Carterocephalus palaemon (Pallas, 1771)}

(I) - UL99/3: 9.6.2006, Primorska, Banjšice, Visoko, $734 \mathrm{~m}, 399943 / 98658 ; 755$ m, 399727/ 98814; hrib Sleme, 769 m, 399542/100466; 18.5. 2006, Primorska, Banjšice, Ježevec, 707 m, 398811/99699; $722 \mathrm{~m}, 399002 / 99718 ; 16.5 .2006$, Primorska, Banjšice, Kuk, 760 m, 400311/99855; UM72/3: 4.6.1998, Primorska, Breginj, Prekopa, 876 m, 378245/126830; VL66/2: 24.5.1994, Notranjska, Loška dolina, Veliki Obrh, $582 \mathrm{~m}$, 462187/61865; VL94/2: 18.5.2011, Dolenjska, dolina Kolpe, Grgelj, 195 m, 494769/42735.
(II) - UM91/3: 14.6.2006, Primorska, Kolovrat, Foni, 569 m, 396901/117862; VL16/3: 13.5.2005, Primorska, Kras, Griže (Sežana), 510 m, 418829/67950; 10.6.2005, Primorska, Kras, Griže (Sežana), 468 m, 418613/68056; VL17/3: 5.5.1996, Primorska, Vipavska brda, Erzelj, 247 m, 415567/76622; VL69/4: 5.6.2001, Dolenjska, Šmarje-Sap, Pucihar, 318 m, 469470/91360; WL17/1: 18.5.2004, Dolenjska, Novo mesto, Ždinja vas, 580 m, 514276/80220; Sela pri Štravber$\mathrm{ku}, 510 \mathrm{~m}, 515023 / 79451$.

(III) - VL16/2: 9.6.1995, Primorska, Sežana, Dol Leskovec, 302 m, 412228/62044.

(ii) - The presence of species in areas which in the Atlas are designated as "white spots": eastern part of Notranjska region, southern part of Dolenjska region.

\section{Thymelicus lineola (Ochsenheimer, 1808)}

(II) - UM83/3: 18.7.2006, Julijske Alpe, Bovec, Črnelski Vršiči, $1740 \mathrm{~m}, 387130 / 136950$; UM83/4: 18.7.2006, Julijske Alpe, Bovec, Pl. Goričica, $1368 \mathrm{~m}$, 388055/135430; $1529 \mathrm{~m}, 387830$ / 136000; UM90/1: 30.6.2006, Primorska, Kanalski Kolovrat, Lig, 489 m, 393760/106520; VL15/ 1: 11.6.2006, Primorska, Lokev, Jirmanec, $421 \mathrm{~m}$, 413383/56889; 25.6.1997, Primorska, Lokev, Mlave, 400 m, 414734/59704; VL77/1: 15.6.2004, Dolenjska, Rašica (Velike Lašče), 480 m, 470524/ 78676; VL84/3: 6.7.2004, Dolenjska, Kočevska Reka, Mokri potok, 525 m, 487702/47269; VM11/4: 17.7.2006, Primorska, Baška grapa, Zakojca, 639 m, 418318/114023; Hudajužna, 424 m, 416263/114968; Obloke, 558 m, 416154/115482; VM14/4: 10.7.2011, Gorenjska, Krma, Zgornja Radovna, 776 m, 418366/143125; VM32/2: 17.7. 2005, Gorenjska, Jelovica, Dražgoše, $800 \mathrm{~m}$, 435206/123986; VM94/2: 18.7.2005 Štajerska, Bele Vode, Senovršnik, 940 m, 495278/142158; WM22/1: 29.6.2005, Štajerska, Frankolovo, Rakova Steza, 330 m, 524113/130770.

(III) - VL16/2: 25.6.1997, Primorska, Sežana, Dol Leskovec, 300 m, 412212/62044; Dane pri Sežani, 350 m, 414584/64790; Filipčje Brdo, 350 m, 414702/65614; VL83/4: 6.7.2004, Dolenjska, Petrina, Grivac, 310 m, 488080/35827.

\section{Thymelicus sylvestris (Poda, 1761)}

(I) - UL99/1: 18.6.2002, Primorska, Goriška Brda, Sabotin, 335 m, 391362/97258; UL99/3: 18.6.2002, Primorska, Grgar, Sv. Gora, $360 \mathrm{~m}$, 396483/96604;22.6.2006, Primorska, Grgar,334 m, 
397698/96967; Zabrdo, 466 m, 398089/98029; 30.6.2006, Primorska, Grgarske Ravne, $460 \mathrm{~m}$, 398089/98030; 25.7.2006, 12.7.2006, Primorska, Banjšice, Visoko, 755 m, 399727/98814; 12.7.2006, Primorska, Banjšice, Visoko, 713 m, 399375/98918; Kuk, 760 m, 400311/99855; 22.6.2006, Primorska, Banjšice, Sleme, 769 m, 399542/100466; 12.7.2006, 22.6.2006, Primorska, Banjšice, Sleme, 773 m, 399549/100511; VL06/1: 11.6.2003, Primorska, Kras, Tublje pri Komnu, 195 m, 402365/70570; Pliskovica, 220 m, 404645/70288; WL07/4: 29. 6.1994, Dolenjska, Novo mesto, Zalog, $178 \mathrm{~m}$, 508548/72226; WL26/1: 28.6.1994, Dolenjska, Gorjanci, Sv. Miklavž, 941 m, 525182/69956.

(II) - UL97/1: 22.6.1997, Primorska, Kras, Lokvica, 172 m, 390622/80672; UM90/4: 30.6. 2006, Primorska, Banjšice, Breščaki, $632 \mathrm{~m}$, 398146/101314; VL07/3: 4.6.2002, Primorska, Kras, Lukovec, 395 m, 408719/77292; VL08/4: 8.7.2002, Primorska, Črniče, Školj Sv. Pavla, 370 m, 407278/85841; VL16/1: 11.6.2003, Primorska, Kras, Tomaj, 340 m, 411137/68931; VL16/4: 11.6.2003, Primorska, Kras, Brestovica pri Povirju, 395 m, 418432/63811; VL68/4: 14.7.2012, Dolenjska, Visoko, 613 m, 467930/82100; VM11/4: 17.7.2006, Primorska, Baška grapa, Zakojca, 639 m, 418318/114023; Srednja vas, 612 m, 417675/ 114396; Obloke, $477 \mathrm{~m}, 416282 / 115279 ; 558 \mathrm{~m}$, 416154/115482; WM12/1: 27.7.1987, 5.7.1988, Štajerska, Ponikovska planota, Marof, $410 \mathrm{~m}$, 512776/129825.

\section{Hesperia comma (Linnaeus, 1758)}

(I) - VL38/4: 4.8.1993, Notranjska, Planinsko polje, Grčarevec, $458 \mathrm{~m}, 439515 / 5081118$.

(II) - VL16/3: 26.9.2005，2.9.2005, Primorska, Kras, Griže (Sežana), 510 m, 418829/67950; 10.6.2005, Primorska, Kras, Griže (Sežana), 468 m, 418613/68056; VL27/4: 22.8.2004, Primorska, Nanos, Laniški vrh, 1040 m, 425555/71520; VL69/4: 26.6.1995, Dolenjska, Ljubljansko barje, Škofljica, 290 m, 466077/92168.

(III) - WM86/1: 23.8.1995, Prekmurje, Petanjci, Gradišče, $194 \mathrm{~m}, 584711 / 167016$; 584615/ 167119; XM15/2: 22.8.1995, Prekmurje, Petišovci, $160 \mathrm{~m}, 611632 / 153802 ; 611274 / 153981$.

\section{Ochlodes sylvanus (Esper, 1777)}

(II) - UM90/3: 16.6.2006, Primorska, Doblar, 130 m, 398812/108738; VL68/4: 14.7.2012, Dolenjska, Visoko, 613 m, 467930/82100; VM41/4: 5.7.2004, Gorenjska, Škofja Loka, Puštal, 380 m, 447303/111248; WM03/1: 18.7.2005, Štajerska,
Šoštanj, Kurja vas, 408 m, 500692/138400; WM 22/1: 29.6.2005, Štajerska, Frankolovo, Rakova Steza, 330 m, 524113/130770; XM05/2: 25.5.1995, Štajerska, Dolnja Bistrica, Kolišče Bobri, 168 m, 601024/154994.

(III) - VL16/2: 25.6.1997, Primorska, Sežana, Dol Leskovec, 300 m, 412212/62044; 9.6.1995, Primorska, Sežana, Dol Leskovec, $302 \mathrm{~m}$, 412228/62044; WM 57/4: 12.6.1996, Štajerska, Sladki Vrh, $232 \mathrm{~m}, 558564 / 172964 ;$ WM76/3: 23.8.1995, Štajerska, Mele, 201 m, 578556/169033; WM86/1: 23.8.1995, Prekmurje, Petanjci, 194 m, $584615 / 167119$.

\section{Zerynthia polyxena (Denis \& Schiffermüller, 1775)}

(I) - UM72/3: 4.6.1998, Primorska, Breginj, Prekopa, 876 m, 378245/126830; VL06/4: 13.4.1995, Primorska, Orlek, Dol Draga, 335 m, 409642/61486; VL23/2: 22.4.2006, Primorska, Rakitovec, Podblatice, $509 \mathrm{~m}, 420443 / 34840$; VL44/4: 21.4.2004, Primorska, Ilirska Bistrica, Sela, 480 m, 449987/41910; 470 m, 449947/41975; VL68/4: 29.4.2003, Dolenjska, Želimlje, $326 \mathrm{~m}$, 468449/83963; VL93/3: 20.5.2004, Dolenjska, dolina Kolpe, Laze pri Predgradu, 550 m, 499332/ 39890.

(II) - UL99/3: 16.5.2006, Primorska, Grgarske Ravne, Zabrdo, 471 m, 397953/98289; Banjšice, Madoni $720 \mathrm{~m}$, 399730/98583; VL13/4: 22.4.2006, Primorska, Rakitovec, $481 \mathrm{~m}, 419757 /$ 34598; Podblatice, $494 \mathrm{~m}, 419993 / 34745$; $498 \mathrm{~m}$, 419900/34890; VL15/1: 11.5.2008, Primorska, Sežana, Lipica, $371 \mathrm{~m}, 412965 / 58830 ; 369 \mathrm{~m}$, 412960/59140; 383 m, 412995/59690; VL37/4: 10.5.2004, Notranjska, Postojna, Zagon, 530 m, $435811 / 72340$.

(III) - VL16/2: 8.4.1995, 5.4.1995, Primorska, Sežana, Dol Leskovec, 310 m, 412167/62026; 13.4.1995, Primorska, Sežana, Dol Leskovec, $305 \mathrm{~m}, 412246 / 62030$.

(i) - New areas of the species distribution: western part of Julian Alps, central part of Slovenia, southeastern part of Notranjska region, southern part of Dolenjska region (Fig. 3). The western part of the Julian Alps (UM72/3) represents the northwestern limit of the species distribution in Slovenia.

\section{Parnassius mnemosyne (Linnaeus, 1758)}

(I) - VL93/3: 18.5.2011, Dolenjska, dolina Kolpe, Spodnja Bilpa, 198 m, 497323/40965. 


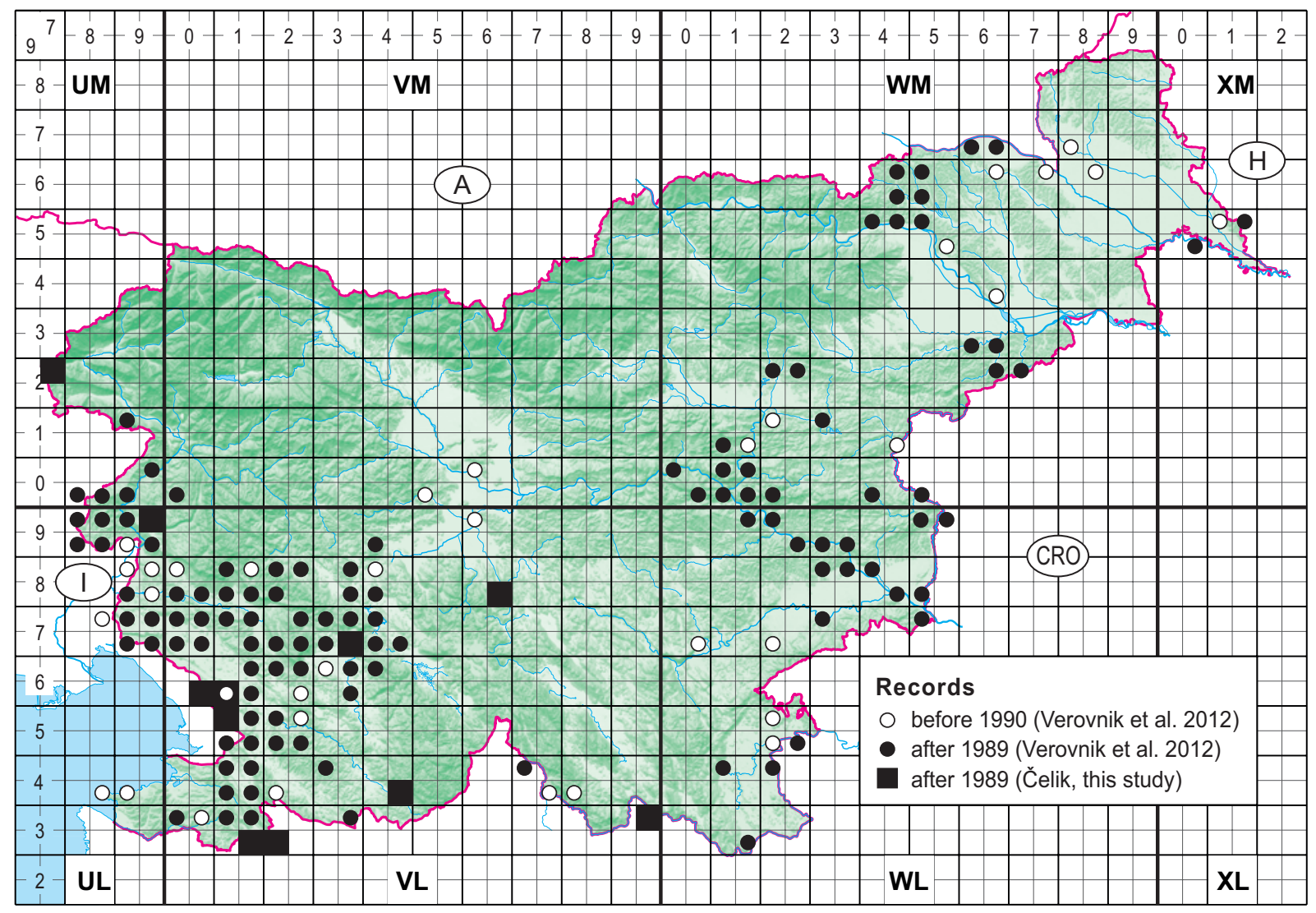

Figure 3: New records on the distribution of Zerynthia polyxena (Denis \& Schiffermüller, 1775) (Čelik, this study) according to the Atlas of butterflies (Lepidoptera: Rhopalocera) of Slovenia (Verovnik et al. 2012: pp. 121).

Slika 3: Novi podatki o razširjenosti vrste Zerynthia polyxena (Denis \& Schiffermüller, 1775) (Čelik, ta študija) glede na podatke v Atlasu dnevnih metuljev (Lepidoptera: Rhopalocera) Slovenije (Verovnik s sod. 2012: str. 121).

(II) - VL19/1: 20.6.2007, Primorska, Vojsko, Gačnik, 919 m, 413545/100100; VL27/4: 21.6.1998, Notranjska, Nanos, Grmada, 1043 m, 425476/ 71546; VL74/3: 19.5.2011, Dolenjska, Bezgarji, 400 m, 477267/46097; VL84/4: 19.5.2011, Dolenjska, Dolnja Briga, 600 m, 486424/41905; Gornja Briga, 620 m, 485837/43203; VL94/2: 18.5.2011, Dolenjska, dolina Kolpe, Grgelj, 195 m, 494769/ 42735; VL99/2: 8.6.2004, Dolenjska, Veliki Gaber, 540 m, 494300/94668; VM82/4: 21.7.2006, Gorenjska, Menina planina, 1415 m, 489305/ 122870; 1376 m, 488888/123100; WL09/2: 26.5. 2004, Dolenjska, Mirna, 380 m, 500714/91089.

(III) - VL26/3: 21.6.1998, Notranjska, Nanos, Pleša, 1236 m, 426709/70224.

(ii) - The presence of species in areas which in the Atlas are designated as "white spots": southern part of Dolenjska region, southern part of Posavsko hills.

\section{Iphiclides podalirius (Linnaeus, 1758)}

(II) - UM90/4: 30.6.2006, Primorska, Banjšice, Breščaki, 632 m, 398146/101314; VL23/2: 22. 4.2006, Primorska, Rakitovec, 509 m, 420443/ 34840; VL94/3: 20.5.2004, Dolenjska, Mozelj, Šibje, 520 m, 497227/48495; VL99/4: 17.5.2011, Dolenjska, Moravška Gora, Červatec, 593 m, 499041/ 96028; 26.5.2004, Dolenjska, Vodice pri Gabrovki, Červatec, 590 m, 499290/95993; Tihaboj, Reber, 590 m, 500025/95803.

(III) - VL69/4: 30.5.1996, Dolenjska, Ljubljansko barje, Škofljica, 290 m, 466196/92275.

\section{Papilio machaon (Linnaeus, 1758)}

(I) - UM72/3: 4.6.1998, Primorska, Breginj, Prekopa, $876 \mathrm{~m}, 378245 / 126830$; VL19/1: 17.7.2006, Primorska, Vojsko, Gačnik, $556 \mathrm{~m}$, 412915/100595; VM54/4: 20.7.2006, Zahodne Karavanke, Košuta, Pl. Spodnje Dolge njive, 1395 m, 455510/143890; Mala Košuta, 1725 m, 456862/144438; 1730 m, 456910/144480. 
(II) - UM83/2: 14.6.2006, Primorska, Bovec, Podčela, 341 m, 385026/131913; UM90/4: 18.5. 2006, Primorska, Banjšice, Lohke, 698 m, 399024/ 102487; VL06/1: 29.5.2002, Primorska, Kras, Pliskovica, 230 m, 404633/70318; VL98/1: 8.6.2004, Dolenjska, Velike Dole pri Šentjurju, $340 \mathrm{~m}$, 493046/89918; VL99/3: 11.7.2003, Dolenjska, Polšnik, Sopota, 690 m, 497957/100781; VL99/4: 8.6.2004, Dolenjska, Moravče pri Gabrovki, Tlaka, 330 m, 498757/93283; VM10/1: 16.6.2006, Primorska, Dolenja Trebuša, Stopnik, 195 m, 413461/106663; VM13/3: 17.7.2009, Julijske Alpe, Pokljuka, Klečica, 1880 m, 418892/139545; Vrh Sedla, 1862 m, 419150/139696; VM60/2: 19.5. 1996, Ljubljana, Vojkova cesta, 298 m, 462956/ 102736; VM72/3: 21.7.2006, Gorenjska, Krivčevo, Volovljek, $1133 \mathrm{~m}$, 475975/127144; VM82/4: 21.7.2006, Gorenjska, Menina planina, Vivodnik, 1423 m, 485860/124290; VM83/1: 18.4.1997, Štajerska, Luče Spodnji Zavratnik, 795 m, 480472/ 137652; WL14/1: 19.5.2004, Bela Krajina, Doblička gora, Vidoše, 390 m, 510683/46745.

(III) - VL74/4: 19.5.2011, Dolenjska, Osilnica, Ribjek, 300 m, 479294/43249.

\section{Leptidea sinapis (Linnaeus, 1758) and L. reali} (Reissinger 1989)

(I) - UM72/3: 4.6.1998, Primorska, Breginj, Prekopa, 876 m, 378245/126830.

(II) - UM83/2: 14.6.2006, Primorska, Bovec, Podčela, $341 \mathrm{~m}$, 385026/131913; UM90/4: 30.6.2006, Primorska, Banjšice, Breščaki, $632 \mathrm{~m}$, 398146/101314; Kanalski Vrh, 350 m, 395765/ 104840; 18.5.2006, Primorska, Banjšice, Lohke, 698 m, 399024/102487; Lužarji, 697 m, 399115/ 103927; VL23/2: 22.4.2006, Primorska, Rakitovec, Podblatice, 509 m, 420443/34840; VL93/3: 1.6. 2004, Dolenjska, Spodnja Bilpa, 200 m, 498960/ 39418; 20.5.2004, Dolenjska, Laze pri Predgradu, Ježuh, 550 m, 499332/39890; VM94/4: 18.7. 2005, Śtajerska, Zavodnje, 713 m, 498950/141288; Mazetov graben, $555 \mathrm{~m}, 500250 / 141326$; Žlebnik, 750 m, 499272/142756; Bele Vode, Srnenski vrh, $952 \mathrm{~m}, 495894 / 141672$; Sleme, $1087 \mathrm{~m}$, 496380/143168; Šentvid pri Zavodnju, $934 \mathrm{~m}$, $498634 / 143192$.

(III) - VM12/2: 25.7.2005, Julijske Alpe, Rodica, Javorje, 1115 m, 412850/123250; VM94/2: 18.7.2005, Štajerska, Bele Vode, Senovršnik, 940 m, 495278/142158; WM77/2: 20.4.1995, Štajerska, Lutverci, 212 m, 572088/172307.

\section{Anthocharis cardamines (Linnaeus, 1758)}

(I) - UM72/3: 4.6.1998, Primorska, Breginj, Prekopa, 876 m, 378245/126830; UM72/4: 4.6. 1998, Primorska, Breginjski kot, Logje, $310 \mathrm{~m}$, 379961/121722; VL23/2: 22.4.2006, Primorska, Rakitovec Podblatice, 509 m, 420443/34840; VL 44/2: 21.4.2004, Primorska, Ilirska Bistrica, Vrbica, 410 m, 444020/44766; Vrbovo, 470 m, 444260/ 45770; VL44/4: 21.4.2004, Primorska, Ilirska Bistrica, Podgraje, $480 \mathrm{~m}, 449987 / 41910 ; 480 \mathrm{~m}$, 450017/42071; VL55/3: 1.4.1994, Notranjska, Loška dolina, Kozarišče, 587 m, 459098/59757; VL 67/4: 22.6.2004, Dolenjska, Podstrmec, Brlog, $610 \mathrm{~m}, 466521 / 72097 ; 600 \mathrm{~m}, 466423 / 72370$; VL 93/3: 20.5.2004, Dolenjska, Laze pri Predgradu, Ježuh, 550 m, 499332/39890; WL06/4: 18.5.2004, Dolenjska, Dolenjske Toplice, Gorenje Sušice, 200 m, 506738/65617; WL08/1: 26.5.2004, Dolenjska, Mirna, Migolska gora, 260 m, 503166/90226.

(II) - UL99/3: 9.6.2006, Primorska, Banjšice, Visoko, 734 m, 399943 /98658; 18.5.2006, Primorska, Grgarske Ravne, 518 m, 397352 /99089; Banjšice, Ježevec, 694 m, 398930/99228; $731 \mathrm{~m}, 398638$ / 99255; 708 m, 398757/99308; 701 m, 398862/99420; 705 m, 398905/99500; 707 m, 398811/99699; Sleme, 773 m, 399549/100511; 16.5.2006, Primorska, Grgarske Ravne, Zabrdo, 471 m, 397953/98289; Banjšice, Madoni, 720 m, 399730/98583; Tlaka, 706 m, 399990/98841; 712 m, 399909/98993; Visoko, 732 m, 399536/98845; 705 m, 399411/98887; $707 \mathrm{~m}, \quad 399466 / 99091 ; \quad 702 \mathrm{~m}, \quad 399593 / 99162$; Kuk, 760 m, 400311/99855; 8.5.2006, Primorska, Grgar, 322 m, 397587/96773; Banjšice, Visoko, 755 m, 399758/98732; Pičulini, 714 m, 399969/ 98817; Tlaka, 715 m, 399413/98946; Sleme, 772 m, 400165/100098; UM83/2: 14.6.2006, Primorska, Bovec, Podčela, 341 m, 385026/131913; UM90/4: 18.5.2006, Primorska, Banjšice, Lohke, $698 \mathrm{~m}$, 399024/102487; Lužarji, 697 m, 399115/103927; UM91/4: 16.6.2006, Primorska, Doblar, Dolina, 344 m, 399024/111763; VL09/1: 16.5.2006, Primorska, Banjšice, Kuk, 748 m, 400350/99650; VL17/2: 8.4.2006, Primorska, Dolenje (Sežana), Mahniči, 290 m, 414766/71844; VL74/3: 19.5.2011, Dolenjska, Bezgarji, 400 m, 477267/46097; VL 79/3: 28.4.1998, Dolenjska, Besnica, Javor, 504 m, 475592/97331; VL84/1: 19.5.2011, Dolenjska, Borovec pri Kočevski Reki, Dragarji, 868 m, 480803/46557; 20.5.2004, Dolenjska, Borovec pri Kočevski Reki, Ravne, 800 m, 482212/46070; VL84/4: 19.5.2011, Dolenjska, Dolnja Briga, Pučki hrib, 560 m, 487513/41700; Dolnja Briga, 600 m, 486424/41905; VL94/3: 20.5.2004, Dolenj- 
ska, Rajndol, 520 m, 496883/46996; Mozelj, Kačji potok, $530 \mathrm{~m}, 497491 / 48104 ; 520 \mathrm{~m}, 497227 / 48495$; WL04/2: 20.5.2004, Dolenjska, Predgrad, Zagozdac, 390 m, 504980/41997; Knežja Lipa, Prerigelj, 680 m, 502909/44999; WL16/1: 18.5.2004, Dolenjska, Novo mesto, Stranska vas, $180 \mathrm{~m}$, 513938/69267; WL25/2: 19.5.2004, Bela Krajina, Metlika, Krivoglavice, 150 m, 522647/54325; WM66/3: 20.4.1995, Štajerska, Gornja Radgona, Apače, 357 m, 569275/170625.

(III) - VL15/1: 11.5.2008, Primorska, Sežana, Lipica, 378 m, 412860/58600; 369 m, 412960/ 59140; 379 m, 412840/59320; VL58/1: 11.5.2001, Notranjska, Ljubljansko barje, Goričica pod Krimom, 286 m, 451046/90593; VM63/4: 5.5.2003, Gorenjska, Kamniška Bistrica, Dom v Kamniški Bistrici, 587 m, 468679/131432; WM77/2: 20.4. 1995, Štajerska, Lutverci, 212 m, 572088/172307.

(ii) - The presence of species in areas which in the Atlas are designated as "white spots": Banjška planota, southeastern part of Notranjska region, western, southern and central part of Dolenjska region.

\section{Aporia crataegi (Linnaeus, 1758)}

(I) - VL23/1: 21.6.2002, Primorska, Rakitovec, Kavčič, 600 m, 420415/36188; VL83/1: 6.7. 2004, Dolenjska, Srobotnik ob Kolpi, $250 \mathrm{~m}$, 485058/38990; VL97/2: 9.6.2004, Dolenjska, Žužemberk, Veliko Lipje, 310 m, 493787/73768; VL98/1: 8.6.2004, Dolenjska, Veliki Gaber, Velike Dole, $310 \mathrm{~m}, 493524 / 90003$.

(II) - UM90/4: 30.6.2006, Primorska, Banjšice, Breščaki, $632 \mathrm{~m}$, 398146/101314; VL16/1: 29.5.2002, Primorska, Kras, Tomaj, $380 \mathrm{~m}$, 411467/68705; 11.6.2003, Primorska, Kras, Tomaj, 340 m, 411137/68931; VL76/4: 22.6.2004, Dolenjska, Ribnica, Nemška vas, 490 m, 478824/64153; 480 m, 478571/64240; VL87/4: 9.6.2004, Dolenjska, Ambrus, Brezovi Dol, 390 m, 489089/75096; VM10/1: 16.6.2006, Primorska, Dolenja Trebuša, Stopnik, 195 m, 413461/106663; VM10/2: 16. 6.2006, Primorska, Dolenja Trebuša, Hotenja, $291 \mathrm{~m}, 412094 / 104768 ; 265 \mathrm{~m}, 411914 / 105006$; Log, 211 m, 413968/105963.

(III) - VL15/3: 25.6.1997, Primorska, Kras, Lokev, 431 m, 415414/57744; VL16/2: 25.6.1997, Primorska, Sežana, Dol Leskovec, 300 m, 412212/ 62044; 9.6.1995, Primorska, Sežana, Dol Leskovec, 302 m, 412228/62044; 25.6.1997, Primorska, Dane pri Sežani, Koblak, 350 m, 414584/64790; Filipčje Brdo, Prjunščine, 350 m, 414702/65614;
VM50/1: 5.7.2004, Gorenjska, Sora, Ločnica, $400 \mathrm{~m}, 450528 / 108375$.

(ii) - The presence of species in areas which in the Atlas are designated as "white spots": central part of Dolenjska region.

\section{Pieris brassicae (Linnaeus, 1758)}

(II) - UL93/4: 2.11.2001, Primorska, Dragonja, Stena, 30 m, 395578/35101; VL16/1: 29.5. 2002, Primorska, Kras, Tomaj, 380 m, 411467/ 68705 ; 11.6.2003, Primorska, Kras, Tomaj, 340 m, 411103/68920; VL16/3: 24.6.2005, 26.9.2005, Primorska, Kras, Griže (Sežana), 360 m, 418626/ 68350; VL16/4: 11.6.2003, Primorska, Kras, Brestovica pri Povirju, 400 m, 418317/63885; VM54/ 4: 20.7.2006, Zahodne Karavanke, Mala Košuta, Pl. Spodnje Dolge njive, 1536 m, 456080/144040; VM72/3: 21.7.2006, Gorenjska, Krivčevo, Rakov rob, 1133 m, 475975/127144; VM82/4: 21.7.2006 , Gorenjska, Menina planina, Goli vrh, $1422 \mathrm{~m}$, 489550/122680; Šavnica, 1415 m, 489305/122870; 1376 m, 488888/123100; Dom na Menini planini, 1455 m, 486386/124184; Vivodnik, 1423 m, 485860/ 124290; VM94/4: 18.7.2005, Štajerska, Zavodnje, Mazetov graben, $555 \mathrm{~m}, 500250 / 141326$.

(III) - VL16/2: 9.6.1995, Primorska, Sežana, Dol Leskovec, $302 \mathrm{~m}$, 412228/62044; VM11/4: 17.7.2006, Primorska, Baška grapa, Zakojca, $612 \mathrm{~m}, 417675 / 114396$.

\section{Pieris mannii (Mayer, 1851)}

(I) - VL69/2: 28.8.2012, Notranjska, Ljubljansko barje, Brest, $289 \mathrm{~m}, 460430 / 93630 ; 289 \mathrm{~m}$, 460515/93655; 286 m, 460420/94110; VL69/3: 27.8. 1996, Gorenjska, Ljubljana, Zg. Kašelj, 275 m, 469560/100300; VM11/4: 17.7.2006, Primorska, Baška grapa, Hudajužna, 424 m, 416263/114968; VM90/4: 23.6.2011, Dolenjska, Renke, Kleviška špica, $340 \mathrm{~m}, 496808 / 104803$.

(II) - WL17/1: 25.4.2004, Dolenjska, Novo mesto, Ždinja vas, 400 m, 513340/78280.

(III) - XM05/3: 19.9.1996, Prekmurje, Kapca, Kot, 164 m, 607266/156248.

(ii) - The presence of species in areas which in the Atlas are designated as "white spots": central part of Slovenia, Cerkljansko hills.

\section{Pieris rapae (Linnaeus, 1758)}

(II) - UM83/3: 18.7.2006, Julijske Alpe, Bovec, Hudi Vršič, 2008 m, 385600/137140; Goričica, $1780 \mathrm{~m}, 387060 / 137280 ; 2115 \mathrm{~m}, 386560 / 137970$; 
Črnelski Vršiči, 2028 m, 386050/137430; 2017 m, 386750/137840; Velika Črnelska špica, $2157 \mathrm{~m}$, 386303/137855; 2320 m, 386162/137990; UM90/1: 30.6.2006, Primorska, Kanalski Kolovrat, Britof, 264 m, 391104/107092; UM90/4: 8.5.2006, Primorska, Banjšice, Mrcinje, $625 \mathrm{~m}, 399598 / 103026$; 30.6.2006, Primorska, Kanalski Vrh, 571 m, 397110/ 104200; VL09/1: 12.7.2006, Primorska, Banjšice, Sveto, 762 m, 400355/100030; VL34/4: 4.4.1994, Notranjska, Ilirska Bistrica, Harije, 484 m, 435695/ 45695; VL99/3: 11.7.2003, Dolenjska, Polšnik, Golek, 690 m, 497957/100781; VM10/2: 16.6.2006, Primorska, Dolenja Trebuša, Hotenja, $291 \mathrm{~m}$, 412094/104768; 265 m, 411914/105006; VM82/4: 21.7.2006, Gorenjska, Menina planina, Goli vrh, 1422 m, 489550/122680; Šavnica, 1415 m, 489305/ 122870; 1376 m, 488888/123100; Dom na Menini planini, $1468 \mathrm{~m}, 486312 / 123800$; Vivodnik, $1423 \mathrm{~m}$, 485860/124290; WL26/1: 28.6.1994, Dolenjska, Gorjanci, Sv. Miklavž, 941 m, 525182/69956.

(III) - VL15/1: 11.6.2006, Primorska, Lokev, Jirmanec, 611 m, 414279/56420; 421 m, 413383/ 56889; 11.5.2008, Primorska, Sežana, Lipica, 375 m, 412890/58715; VM73/2: 30.7.2008, Kamniško-Savinjske Alpe, Dleskovec, Vrtače, $1800 \mathrm{~m}$, 475250/134090; 29.7.2008, Kamniško-Savinjske Alpe, Veliki Vrh, Molička planina, $1770 \mathrm{~m}$, 474240/134940; VM94/2: 18.7.2005, Štajerska, Bele Vode, Senovršnik, 940 m, 495278/142158; WM77/2: 20.4.1995, Štajerska, Lutverci, 212 m, $572088 / 172307$.

(ii) - The presence of species in areas which in the Atlas are designated as "white spots": Banjška planota.

\section{Pieris ergane (Geyer, 1828)}

(I) - VL04/2: 30.7.1996, Primorska, Koper, Vanganel, $60 \mathrm{~m}, 404915 / 41401$.

\section{Pieris napi (Linnaeus, 1758)}

(II) - VL13/4: 22.4.2006, Primorska, Rakitovec, Podblatice, $481 \mathrm{~m}, 419757 / 34598 ; 494 \mathrm{~m}$, 419993/34745; 498 m, 419900/34890; Maršiči, 125 m, 415367/34988; VL15/1: 11.5.2008, Primorska, Sežana, Lipica, 369 m, 412960/59140; VL23/ 2: 22.4.2006, Primorska, Rakitovec, Podblatice, 509 m, 420443/34840; VL84/4: 19.5.2011, Dolenjska, Dolnja Briga, Pučki hrib, 560 m, 487513/ 41700; 600 m, 486424/41905; VL94/2: 6.7.2004, Dolenjska, Podstene pri Kostelu, Dolenji Potok, 300 m, 491880/41854; VL94/3: 20.5.2004, Dolenjska, Mozelj, Kačji potok, 530 m, 497491/48104;
VL99/4: 17.5.2011, Dolenjska, Moravška Gora, Červatec, 593 m, 499041/96028; 26.5.2004, Dolenjska, Moravška Gora, Červatec, 590 m, 499290/ 95993; 8.6.2004, Dolenjska, Gabrovka, Tlaka, 330 m, 498757/93283; WL16/1: 18.5.2004, Dolenjska, Novo mesto, Rajnovšče, 180 m, 513938/69267.

(III) - VL83/4: 6.7.2004, Dolenjska, Petrina, Grivački jarek, 310 m, 488080/35827; WM76/3: 23.8.1995, Štajerska, Mele, 201 m, 578556/169033; WM77/2: 20.4.1995, Štajerska, Lutverci, 212 m, $572088 / 172307$.

\section{Pieris bryoniae (Hübner, 1806)}

(II) - VM53/2: 21.7.2010, Kamniško-Savinjske Alpe, Storžič, $1625 \mathrm{~m}, 453907 / 133783 ; 1612 \mathrm{~m}$, 453730/133838; 1570 m, 453448/133846; Škarjev rob, $1850 \mathrm{~m}, 454780 / 134630$.

\section{Pontia edusa (Fabricius, 1777)}

(I) - VL37/3: 30.7.1993, Notranjska, Planinsko polje, Hotenjka, 445 m, 440164/80762; 4.8. 1993 Notranjska, Planinsko polje, Hotenjka, 445 m, 439762/80942; VL47/1: 16.6.1993, Notranjska, Planinsko polje, Podgora, $447 \mathrm{~m}, 441858$ / 79043; 6.9.1993, Notranjska, Planinsko polje, Log, 447 m, 442222/79453; 20.8.1993, Notranjska, Planinsko polje, Jakovski grič, 458 m, 442605/79675; VL68/3: 3.7.2009, Dolenjska, Pijava Gorica, Rogovila, $295 \mathrm{~m}, 466680 / 88550$.

(II) - VL14/3: 10.9.1995, Primorska, Petrinje, Cesarska vala, 401 m, 415565/48897; VL59/4: 18.9 . 2012 Dolenjska, Ljubljansko barje, Brest, $288 \mathrm{~m}$, 460265/93735; VL69/2: 25.5.2012, Dolenjska, Ljubljansko barje, Brest, 289 m, 460430/93630; 460515/93655.

(ii) - The presence of species in areas which in the Atlas are designated as "white spots": central part of Notranjska region.

\section{Colias crocea (Geoffroy, 1785)}

(I) - VL06/1: 12.10.2004, Primorska, Kras, Podbreg, 225 m, 404678/70300; VL19/1: 17.7. 2006, Primorska, Vojsko, Gačnik, 556 m, 412915/ 100595.

(II) - UL99/3: 22.9.2006, Primorska, Banjšice, Visoko, 755 m, 399727/98814; 12.7.2006, 22.9. 2006, Primorska, Banjšice, Tlaka, 705 m, 399466/ 99091; 22.6.2006 , 25.7.2006, 23.8.2008, 5.9.2006, 22.9.2006, Primorska, Banjšice, Tlaka, $702 \mathrm{~m}$, 399593/99162; 12.7.2006, 23.8.2006, 22.9.2006, Primorska, Banjšice, Sleme, 769 m, 399542/100466; 22.9.2006, Primorska, Banjšice, Sleme, 773 m, 
399549/100511; UM72/3: 4.6.1998, Primorska, Breginj, Prekopa, 876 m, 378245/126830; UM83/ 3: 18.7.2006, Julijske Alpe, Bovec, Goričica, 1740 m, 387130/136950; UM90/4: 30.6.2006, Primorska, Kanalski Vrh, $571 \mathrm{~m}, 397110 / 104200$; VL76/4: 22.6.2004, Dolenjska, Ribnica, Ugar, 480 m, 478571/64240; VM40/1: 5.7.2004, Gorenjska, Ožbolt nad Zmincem, Kartel, 710 m, 444323/ 108159; VM53/2: 21.7.2010, Kamniško-Savinjske Alpe, Storžič, 1625 m, 453907/133783; VM54/4: 20.7.2006, Zahodne Karavanke, Mala Košuta, 1725 m, 456862/144438; 1730 m, 456910/144480; VM72/3: 21.7.2006, Gorenjska, Krivčevo, Rakov rob, 1133 m, 475975/127144; 30.7.2008, Kamniško-Savinjske Alpe, Črni vrh, Prag, $1800 \mathrm{~m}$, 474660/134250; WL07/4: 29.6.1994, Dolenjska, Novo mesto, Zalog, 163 m, 509502/72582; WL08/ 1: 26.5.2004, Dolenjska, Mirna, Migolska gora, $260 \mathrm{~m}, 503166 / 90226$.

(III) - XM05/3: 19.9.1996, Prekmurje, Kapca, Kot, 164 m, 607266/156248.

(ii) - The presence of species in areas which in the Atlas are designated as "white spots": Banjška planota, Idrijsko hills.

\section{Colias hyale (Linnaeus, 1758)}

(I) - VL03/1: 14.6.2002, Primorska, Koprska Brda, Koštabona, 100 m, 402217/37719; WM00/3: 27.7.2010, 14.9.2010, Štajerska, Hrastnik, ob Savi, $207 \mathrm{~m}, 506947 / 108567$.

(II) - VL08/4: 8.7.2002, Primorska, Črniče, Školj Sv. Pavla, 370 m, 407278/85841; WM12/1: 27.7.1987, Štajerska, Ponikovska planota, Marof, $410 \mathrm{~m}, 512776 / 129825$.

(III) - WM10/1: 14.9.2010, Štajerska, Suhadol (Laško), Dular, 237 m, 511593/106375; 225 m, 511395/106425; $255 \mathrm{~m}, 511518 / 106476 ; 250 \mathrm{~m}$, $511456 / 106500$.

(ii) - The presence of species in areas which in the Atlas are designated as "white spots": Koprska Brda region, Posavsko hills.

(iv) - New information on species occurrence in Vipava valley: species distribution map in the Atlas (Verovnik et al. 2012: pp. 161) indicates that in this region the species was last recorded before year 1990. My record from the surroundings of Črniče (VL08/4) shows that in Vipava valley species was also found in the last decade.

\section{Colias alfacariensis (Ribbe, 1905)}

(I) - UM72/3: 4.6.1998, Primorska, Breginj,
Prekopa, 876 m, 378245/126830; UM90/4: 18.5. 2006, Primorska, Banjšice, Lohke, 698 m, 399024/ 102487.

(II) - VL15/1: 11.6.2006, Primorska, Lokev, Jirmanec, $658 \mathrm{~m}, 414621 / 56055$; 11.5.2008, Primorska, Lipica (Sežana), 380 m, 412825/59490; VM82/4: 21.7.2006, Gorenjska, Menina planina, Šavnica, 1415 m, 489305/122870.

(iv) - New information on the vertical distribution of species: according to the data in the Atlas (Verovnik et al. 2012: pp. 162), the species occurs at heights up to $1400 \mathrm{~m}$ a. s. l. only in the Julian Alps. My record from Menina planina (VM82/4) shows that species occurs at the upper limit of montane zone (i.e. at altitudes above $1400 \mathrm{~m}$ ) also in the Kamniško-Savinjske Alps.

\section{Gonepteryx rhamni (Linnaeus, 1758)}

(II) - UM83/3: 18.7.2006, Julijske Alpe, Bovec, Hudi Vršič, 2008 m, 385600/137140; UM91/3: 14.6.2006, Primorska, Kolovrat, Foni, $569 \mathrm{~m}$, 396901/117862; Kamno, 173 m, 395606/119343; VL13/4: 22.4.2006, Primorska, Rakitovec, $481 \mathrm{~m}$, 419757/34598; Podblatice, 494 m, 419993/34745; 498 m, 419900/34890; Maršiči (Koper), 125 m, 415367/34988; VL16/1: 11.6.2003, Primorska, Kras, Tomaj, $340 \mathrm{~m}$, 411137/68931; VL23/2: 22.4.2006, Primorska, Rakitovec, Podblatice, 509 m, 420443/34840; 21.6.2002, Primorska, Rakitovec, Ubred, 660 m, 421031/36003; VL56/2: 27.4.1994, Notranjska, Cerkniško polje, Otok, 568 m, 451970/65778; VL83/1: 6.7.2004, Dolenjska, Srobotnik ob Kolpi, Rački potok, $250 \mathrm{~m}$, 485058/38990; VL83/4: 6.7.2004, Dolenjska, Petrina, Grivački jarek, 310 m, 488080/35827; VM10/2: 16.6.2006, Primorska, Dolenja Trebuša, Hotenja, $291 \mathrm{~m}, 412094 / 104768 ; 265 \mathrm{~m}$, 411914/105006; VM11/4: 17.7.2006, Primorska, Baška grapa, Srednja vas, 639 m, 418318/114023; $612 \mathrm{~m}, 417675 / 114396$; Hudajužna, $424 \mathrm{~m}$, 416263/114968; VM12 /2: 25.7.2005, Julijske Alpe, Rodica, Pl. Suha, 1388 m, 412041/122509; VM73/2: 30.7.2008, Kamniško-Savinjske Alpe, Dleskovec, Vrtače, 1800 m, 475250/134090; Beli vrh, 1880 m, 473150/134790; WL03/3: 20.5.2004, Dolenjska, Stari trg ob Kolpi, Žlebine, 280 m, 505883/38597.

(III) - VM94/2: 18.7.2005, Štajerska, Bele Vode, Senovršnik, 940 m, 495278/142158; WM 77/2: 17.4.1996, Štajerska, Gornja Radgona, Podgrad, 209 m, 575214/171401; 20.4.1995, Štajerska, Lutverci, 212 m, 572088/172307. 


\section{Hamearis lucina (Linnaeus, 1758)}

(I) - UM90/4: 30.6.2006, Primorska, Banjšice, Bate, 597 m, 397290/101950; VL84/4: 6.7.2004, Dolenjska, Morava, Suhor, 530 m, 490041/43757.

(II) - VL15/1: 11.5.2008, Primorska, Sežana, Lipica, 371 m,412965/58830;369 m,412960/59140; VL27/4: 21.6.1998, Notranjska, Nanos, Grmada, 1043 m, 425476/71546; VL45/4: 9.7.1994, Notranjska, Koritnice, Suha reber, 671 m, 445539/51767; VL67/3: 15.6.2004, Dolenjska, Rašica, Dolščaki, 500 m, 467358/78088; VL79/3: 28.4.1998, Dolenjska, Besnica, Javor, 504 m, 475592/97331.

(III) - WM77/2: 20.4.1995, Štajerska, Lutverci, $212 \mathrm{~m}$, 572088/172307.

(ii) - The presence of species in areas which in the Atlas are designated as "white spots": Banjška planota, central part of Slovenia, western part of Dolenjska region.

\section{Lycaena phlaeas (Linnaeus, 1761)}

(II) - UL99/3: 9.6.2006, Primorska, Grgar, Grašišče, 312 m, 397513/96420; 22.9.2006, Primorska, Banjšice, Tlaka, 702 m, 399593/99162; 25.7.2006, Primorska, Banjšice, Visoko, $700 \mathrm{~m}$, 399582/99220; 22.9.2006, Primorska, Banjšice, Sleme, 769 m, 399542/100466; VL25/2: 4.5.2009, Notranjska, Brkini, Naklo, 409 m, 423045/55833; 22.4.2009, Notranjska, Brkini, Naklo, $410 \mathrm{~m}$, 423026/55885; 11.4.2009, Notranjska, Brkini, Naklo, 415 m, 423064/55933; VL69/4: 20.6.1996, Dolenjska, Ljubljansko barje, Škofljica, $289 \mathrm{~m}$, 465920/92155; 30.5.1996, Dolenjska, Ljubljansko barje, Škofljica, 290 m, 466196/92275; VM40/4: 23.6.2004, Gorenjska, Polhograjsko hribovje, Mačkov graben, 460 m, 447560/104250; VM53/2: 21.7.2010, Kamniško-Savinjske Alpe, Storžič, 1625 m, 453907/133783; Psica, 1612 m, 453730/ 133838; WL07/4: 29.6.1994, Dolenjska, Novo mesto, Zalog, 163 m, 509502/72582; 28.6.1994, Dolenjska, Novo mesto, Zalog, 164 m, 509644/72992.

\section{Lycaena dispar (Haworth, 1802)}

(I) - VL93/1: 6.7.2004, Dolenjska, Kostel, Mavrc, 210 m, 493418/39581.

(III) - VM04/1: 18.6.2012, Julijske Alpe, Podkoren, Zelenci, 835 m, 403610/150465.

(iv) - New information on species occurrence in Alps: Zelenci at the source of the Sava river is the only known locality of the species in the Alps. The species has not been observed in this region since 1990 (Verovnik et al. 2012: pp. 173). Accord- ing to the authors' comments (cf. Verovnik et al. 2012: pp. 172), the species is completely absent from the Alps. I observed the species at the locality Zelenci (VM04/1) in 2012 meaning that: (a) the species is still present in the Alps; (b) it occurs at altitudes above $600 \mathrm{~m}$ (cf. Verovnik et al. 2012: pp. 172); (c) its range in Slovenia extends to the most northwestern limit of the country.

\section{Lycaena virgaureae (Linnaeus, 1758)}

(I) - UL99/3: 12.7.2006, Primorska, Banjšice, Visoko, 755 m, 399727/98814.

(ii) - The presence of species in areas which in the Atlas are designated as "white spots": Banjška planota.

\section{Lycaena tityrus (Poda, 1761)}

(I) - VM53/2: 21.7.2010, Kamniško-Savinjske Alpe, Storžič, Škarjev rob, 1745 m, 454764/135115.

(II) - UL99/3: 9.6.2006, Primorska, Banjšice, Visoko, 755 m, 399727/98814; VL16/1: 29.5.2002, Primorska, Kras, Tomaj, 340 m, 411141/68960; VL67/3: 22.6.2004, Dolenjska, Rašica, Dolščaki, 500 m, 467402/78160; VL69/3: 27.8.1996, Gorenjska, Ljubljana, Zg. Kašelj, 275m, 469560/100300; VL79/2: 12.7.2003, Dolenjska, Dobje, Črna dolina, 350 m, 473810/92850; VM10/2: 16.6.2006, Primorska, Dolenja Trebuša, Hotenja, $291 \mathrm{~m}$, 412094/104768; 265 m, 411914/105006; 16.6.2006 Primorska, Stopnik, Utrška grapa, 211 m, 413968/ 105963.

(III) - VM40/4: 23.6.2004, Gorenjska, Polhograjsko hribovje, Mačkov graben, 460 m, 447560/ 104250.

(iv) - New information on the vertical distribution of species: according to the data in the Atlas (Verovnik et al. 2012: pp. 176), the species occurs at heights up to $1800 \mathrm{~m}$ a. s. l. only in the Julian Alps. My record from Mt. Storžič (VM53/2) shows that species occurs within the submontane zone also in the Kamniško-Savinjske Alps.

\section{Lycaena alciphron (Rottemburg, 1775)}

(I) - VL15/3: 25.6.1997, Primorska, Kras, Lokev, $431 \mathrm{~m}, 415414 / 57744$.

(II) - UL99/3: 22.6.2006, Primorska, Grgar, Grič, 466 m, 398089/98029; 22.6.2006, Primorska, Banjšice, Visoko, 755 m, 399727/98814; 713 m, 399375/98918; Kuk, 760 m, 400311/99855; VL68/3: 6.7.2008, Dolenjska, Pijava Gorica, Rogovila, $295 \mathrm{~m}, 466680 / 88550$. 
(ii) - The presence of species in areas which in the Atlas are designated as "white spots": Banjška planota, Kras.

\section{Lycaena hippothoe (Linnaeus, 1761)}

(II) - UL99/3: 22.6.2006, Primorska, Banjšice, Visoko, 702 m, 399593/99162; UM90/4: 30.6. 2006, Primorska, Kanalski Vrh, 571 m, 397110/ 104200; VL37/4: 12.7.2004, Notranjska, Postojna, Mali Otok, $520 \mathrm{~m}, 436228 / 71451$.

(III) - VL69/3: 3.6.1996, Gorenjska, Ljubljana, Zg. Kašelj, 275 m, 469557/100312.

(ii) - The presence of species in areas which in the Atlas are designated as "white spots": Banjška planota.

\section{Favonius quercus (Linnaeus, 1758)}

(II) - UL97/1: 29.6.2006, Primorska, Kras, Lokvica, 175 m, 390690/80620; VL45/4: 9.7.1994, Notranjska, Koritnice, Suha reber, 671 m, 445539/ 51767.

\section{Callophrys rubi (Linnaeus, 1758)}

(I) - UM72/3: 4.6.1998, Primorska, Breginj, Prekopa, 876 m, 378245/126830; VL23/2: 22.4 . 2006, Primorska, Rakitovec, Podblatice, 509 m, $420443 / 34840$.

(II) - UL99/3: 8.5.2006, Primorska, Banjšice, 755 m, 399758/98732; 16.5.2006, 9.6.2006, Primorska, Banjšice, Visoko, 755 m, 399727/98814; 16.5. 2006, Primorska, Banjšice, Visoko, 732 m, 399536/ 98845; 705 m, 399411/98887; 707 m, 399466/99091; 16.5.2006, Primorska, Banjšice, Tlaka, $712 \mathrm{~m}$, 399909/98993; 8.5.2006, Primorska, Banjšice, Tlaka, 705 m, 399485/99089; 18.5.2006, Primorska, Banjšice, Ježevec, $731 \mathrm{~m}, 398638 / 99255 ; 708 \mathrm{~m}$, 398757/99308; Pičulini, 715 m, 399936/99304; 16.5.2006, Primorska, Banjšice, Kuk, $760 \mathrm{~m}$, 400311/99855; 8.5.2006, Primorska, Banjšice, Sveto, $772 \mathrm{~m}, 400165 / 100098$; 18.5.2006, Primorska, Banjšice, Sleme, 769 m, 399542/100466; 773 m, 399549/100511; 8.5.2006, Primorska, Banjšice, Sleme, 791 m, 399062/100908; VL09/1: 16.5.2006, Primorska, Banjšice, Kuk, 748 m, 400350/99650; VL04/2: 28.4.1994, Primorska, Koper, Škocjanski zatok, 0 m, 403178/45684; VL13/4: 22.4.2006, Primorska, Rakitovec, 481 m, 419757/34598; Podblatice, 498 m, 419900/34890; VL99/4: 26.5.2004, Dolenjska, Moravška gora, Červatec, $590 \mathrm{~m}$, $499290 / 95993$.

(III) - VL15/1: 11.5.2008, Primorska, Sežana, Lipica, 379 m, 412840/59320; WL27/2: 18.5.2004,
Dolenjska, Velike Brusnice, Tolsti vrh, $300 \mathrm{~m}$, 521990/74163.

\section{Satyrium w-album (Knoch, 1782)}

(I) - VM40/3: 5.7.2004, Gorenjska, Škofja Loka, Hrastnica, 535 m, 446363/107359.

(II) - VL68/3: 12.7.2009, Dolenjska, Pijava Gorica, Rogovila, 295 m, 466620/88615.

\section{Satyrium pruni (Linnaeus, 1758)}

(I) - UM91/4: 16.6.2006, Primorska, Doblar, Avško, 655 m, 397757/111482; VM11/4: 17.7.2006, Primorska, Baška grapa, Obloke, 558 m, 416154/ 115482.

(ii) - The presence of species in areas which in the Atlas are designated as "white spots": the lower part of the Soča river valley, southeastern part of Julian Alps.

\section{Satyrium spini (Denis \& Schiffermüller, 1775)}

(I) - VL16/3: 10.6.2005, Primorska, Kras, Griže, $510 \mathrm{~m}, 418829 / 67950$; Dobravce, $468 \mathrm{~m}$, 418613/68056; 24.6.2005, Primorska, Kras, Griže, 460 m, 418604/68099; VL38/4: 16.7.1993, 30.7.1993, Notranjska, Planinsko polje, Lanski vrh, $456 \mathrm{~m}$, 435585/81108; VM94/4: 18.7.2005, Štajerska, Bele Vode, Srnenski vrh, $952 \mathrm{~m}, 495894 / 141672$; WM23/2: 29.6.2005, Štajerska, Lipa pri Frankolovem, Zgornje Selce, 659 m, 522566/134766.

(III) - VM32/4: 17.7.2005, Gorenjska, Jelovica, Dražgoše, 1020 m, 438000/123300.

(ii) - The presence of species in areas which in the Atlas are designated as "white spots": central part of Notranjska region, Velenjsko hills.

\section{Satyrium ilicis (Esper, 1779)}

(II) - UL99/3: 25.7.2006, Primorska, Banjšice, Kuk, 760 m, 400311/99855; VL09/2: 18.6.2002, Primorska, Ajševica, Lijak, 100 m, 401138/91333.

(III) - VL16/2: 25.6.1997, Primorska, Sežana, Dol Leskovec, 300 m, 412212/62044.

\section{Satyrium acaciae (Fabricius, 1787)}

(I) - VL67/2: 19.7.2009, Notranjska, Bloška planota, Bloški hrib, 740 m, 461260/71927.

(II) - VL46/2: 12.6.2003, Notranjska, Trnje (Pivka), Debeli vrh, $640 \mathrm{~m}, 442256 / 62620$; VL84/1: 26.6.1994, Dolenjska, Kočevska Reka, $525 \mathrm{~m}, 484033 / 48042$.

(ii) - The presence of species in areas which 
in the Atlas are designated as "white spots": eastern part of Notranjska region.

\section{Leptotes pirithous (Linnaeus, 1767)}

(I) - VL44/1: 2.8.1994, Primorska, Ilirska Bistrica, Trnovšek, 400 m, 440456/48444.

(II) - UL93/4: 2.11.2001, Primorska, Dragonja, Stena, $30 \mathrm{~m}$, 395578/35101.

\section{Cupido minimus (Fuessly, 1775)}

(I) - WL26/1: 28.6.1994, Dolenjska, Gorjanci, Sv. Miklavž, 941 m, 525182/69956.

(II) - UM83/3: 18.7.2006, Julijske Alpe, Bovec, Črnelski Vršiči, 1740 m, 387130/136950; 1780 m, 387060/137280; 2115 m, 386560/137970; UM90/4: 18.5.2006, Primorska, Banjšice, Lužarji, 697 m, 399115/103927; VL15/1: 11.6.2006, Primorska, Lokev, Jirmanec, 658 m, 414621/56055; VL47/1: 11.5.1993, Notranjska, Planinsko polje, Jakovski grič, 464 m, 442830/79620; VM33/2: 7.5.1996, Gorenjska, Radovljica, Cajhnova žaga, $415 \mathrm{~m}$, 435312/133420; VM54/4: 20.7.2006, Zahodne Karavanke, Mala Košuta, 1725 m, 456862/144438; 1730 m, 456910/144480; VM73/2: 29.7.2008, Kamniško-Savinjske Alpe, Veliki Vrh, Molička planina, 1770 m, 474240/134940; VM82/4: 21.7.2006, Gorenjska, Menina planina, Šavnica, $1422 \mathrm{~m}$, 489550/122680; $1415 \mathrm{~m}, 489305 / 122870 ; 1376 \mathrm{~m}$, 488888/123100; VM94/2: 18.7.2005, Štajerska, Bele Vode, Senovršnik, 940 m, 495278/142158; WM23/2: 29.6.2005, Štajerska, Straža pri Novi Cerkvi, Zg. Razdelj, 410 m, 522771/131829.

(III) - VL16/2: 9.6.1995, Primorska, Sežana, Dol Leskovec, 302 m, 412228/62044; 25.6.1997, Primorska, Filipčje Brdo, Prjunščine, $350 \mathrm{~m}$, 414702/65614; XM15/2: 22.8.1995, Prekmurje, Petišovci, $160 \mathrm{~m}, 611632 / 153802$.

\section{Cupido argiades (Pallas, 1771)}

(I) - VL47/1: 6.9.1993, Notranjska, Planinsko polje, Lomi, 446 m, 442030/79150; 20.8.1993, Notranjska, Planinsko polje, Loka, 444 m, 441683/ 79500; 12.8.1993, Notranjska, Planinsko polje, Sv. Jedert, 444 m, 440450/80203.

(II) - VL15/1: 11.5.2008, Primorska, Sežana, Lipica, 379 m, 412840/59320; VL25/2: 25.4.2009, Notranjska, Brkini, Sušica, 403 m, 422745/55941; VM71/2: 22.8.2003, Gorenjska, Radomlje, Rova, $337 \mathrm{~m}, 471304 / 114890$; $339 \mathrm{~m}, 471277 / 115029$; WM86/4: 4.5.1995, Štajerska, Vučja vas, 185 m, $585857 / 162473$.

(ii) - The presence of species in areas which in the Atlas are designated as "white spots": central part of Notranjska region.

\section{Cupido alcetas (Hoffmannsegg, 1804)}

(I) - UM91/4: 16.6.2006, Primorska, Doblar, Lepenka, 344 m, 399024/111763; WM03/1: 18.7.2005, Štajerska, Šoštanj, Kurja vas, 408 m, 500692/138400; WM23/2: 29.6.2005, Štajerska, Straža pri Novi Cerkvi, Zg. Razdelj, 410 m, 522771/131829; Straža pri Dolu, Jesenica, 341 m, 524180/132467; Lipa pri Frankolovem, Zgornje Selce, 659 m, 522566/134766.

(II) - UL99/3: 12.7.2006, Primorska, Banjšice, Visoko, 713 m, 399375/98918.

(ii) - The presence of species in areas which in the Atlas are designated as "white spots": Velenjsko hills.

\section{Celastrina argiolus (Linnaeus, 1758)}

(I) - VL99/4: 26.5.2004, Dolenjska, Moravška gora, Červatec, 590 m, 499290/95993; VM13/3: 17.7.2009, Julijske Alpe, Pokljuka, Klečica, 1862 m, 419150/139696; VM40/3: 5.7.2004, Gorenjska, Škofja Loka, Hrastnica, 500 m, 446728/107803; WL09/2: 17.5.2011, Dolenjska, Brezovo, Brša, $630 \mathrm{~m}, 500731 / 95748$.

(II) - UM90/4: 30.6.2006, Primorska, Kanal, Morsko, $350 \mathrm{~m}$, 395765/104840; VL15/1: 11.5.2008, Primorska, Sežana, Lipica, 369 m, 412960/59140; VL67/2: 16.7.1994, Notranjska, Bloška planota, Staro Volčje, 734 m, 461134/72226.

(III) - VL16/2: 13.4.1995, Primorska, Sežana, Dol Leskovec, 305 m, 412246/62030.

(ii) - The presence of species in areas which in the Atlas are designated as "white spots": northeastern part of Julian Alps, Polhograjsko hills, Posavsko hills.

(iv) - New information on the vertical distribution of species: according to the data in the Atlas (Verovnik et al. 2012: pp. 214), the species occurs in the Karavanke Mountains and Julian Alps at heights up to $1300 \mathrm{~m}$ a. s. 1 . My record from Pokljuka (VM13/3) shows that species is present in the Julian Alps also within submontane zone, i.e. between 1800-1900 m a. s. 1 .

\section{Pseudophilotes vicrama (Moore, 1865)}

(I) - UL93/4: 25.4.1987, Primorska, Dragonja, Stena, 33 m, 395578/35101.

(II) - UL99/3: 25.7.2006, Primorska, Banjšice, Sleme, 769 m, 399542/100466; VL13/4: 
22.4.2006, Primorska, Rakitovec, Obešen hrib, 501 m, 419174/34930; VL17/1: 5.5.1996, Primorska, Kras, Štanjel, 320 m, 410821/76047; Braniška dolina, Ječmenov hrib, 138 m, 410619/77066; VL18/1: 27.6.2002, Primorska, Lokavec, Predmeja, 710 m, 412810/88198; VL25/2: 11.5.2009, Notranjska, Brkini, Sušica, $401 \mathrm{~m}, 422722 / 55935$; 4.5.2009, Notranjska, Brkini, Sušica, $410 \mathrm{~m}$, 422956/55951; VL47/4: 27.7.1994, Notranjska, Cerknica, Kamna Gorica, 620 m, 450339/73257.

(ii) - The presence of species in areas which in the Atlas are designated as "white spots": Banjška planota, western part of Koprska Brda region.

\section{Scolitantides orion (Pallas, 1771)}

(I) - UL93/4: 30.7.1996, Slovenija, Primorska, Dragonja, Stena, 33 m, 395578/35101; UM72/4: 4.6.1998, Primorska, Breginjski kot, Logje, 535 m, 379515/122553.

(II) - VL16/3: 13.5.2005, Primorska, Kras, Griže, 510 m, 418829/67950; VL25/2: 18.5.2009,
Notranjska, Brkini, Sušica, 382 m, 422388/56022; VL97/2: 9.6.2004, Dolenjska, Suha krajina, Veliko Lipje, 310 m, 493787/73768; VM90/1:11.7.2003, Štajerska, Zagorje ob Savi, Mošenik, $290 \mathrm{~m}$, 494192/106080; WL17/1: 18.5.2004, Slovenija, Dolenjska, Novo mesto, Sela pri Štravberku, $510 \mathrm{~m}, 515023 / 79451$.

(ii) - The presence of species in areas which in the Atlas are designated as "white spots": western part of Koprska Brda region.

\section{Glaucopsyche alexis (Poda, 1761)}

(I) - UM83/2: 14.6.2006, Primorska, Bovec, Podčela, 343 m, 384910/131553; WM52/4: 22.5. 1998, Štajerska, Donačka Gora, Sv. Donat, 500 m, $557605 / 124019$.

(II) - UL99/3: 16.5.2006, Primorska, Banjšice, Visoko, 732 m, 399536/98845; VL09/1: 16.5. 2006, Primorska, Banjšice, Kuk, 748 m, 400350/ 99650; UM90/4: 18.5.2006, Primorska, Banjšice, Lužarji, 697 m, 399115/103927; UM91/4: 16.6.

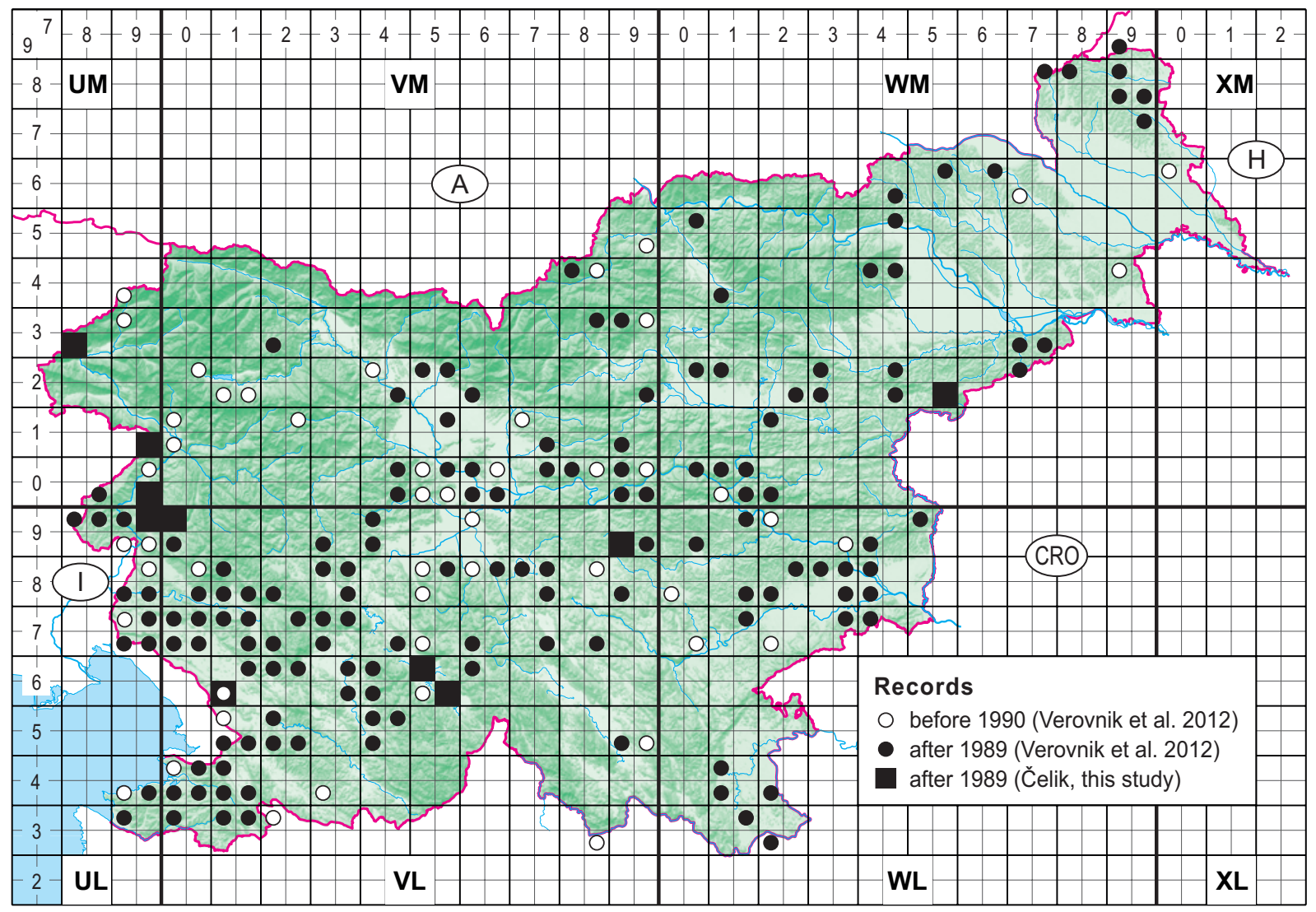

Figure 4: New records on the distribution of Glaucopsyche alexis (Poda, 1761) (Čelik, this study) according to the Atlas of butterflies (Lepidoptera: Rhopalocera) of Slovenia (Verovnik et al. 2012: pp. 221).

Slika 4: Novi podatki o razširjenosti vrste Glaucopsyche alexis (Poda, 1761) (Čelik, ta študija) glede na podatke v Atlasu dnevnih metuljev (Lepidoptera: Rhopalocera) Slovenije (Verovnik s sod. 2012: str. 221). 
2006, Primorska, Kanalski Kolovrat, Avško, 655 m, 397757/111482; VL56/1: 25.5.1994, Notranjska, Cerkniško polje, Otok, $570 \mathrm{~m}, 451757 /$ 66286; VL56/4: 24.5.1994, Notranjska, Loška dolina, Dane, $568 \mathrm{~m}, 457111 / 62552$; VL99/2: 8.6.2004, Dolenjska, Veliki Gaber, Mišji potok, $330 \mathrm{~m}, 493079 / 92321$.

(III) - VL16/2: 9.6.1995, Primorska, Sežana, Dol Leskovec, 302 m, 412228/62044.

(i) and (ii) - The presence of species in areas which in the Atlas are designated as "white spots": western part of Julian Alps, the lower part of the Soča river valley, Banjška planota. The western part of the Julian Alps represents the northwestern limit of the species distribution in Slovenia.

(iv) - New information on species occurrence in NW Slovenia: the species distribution map in the Atlas (Verovnik et al. 2012: pp. 221) indicates that for a large region, including the Julian Alps, the lower part of the Soča river valley, Banjška planota, Idrijsko hills and Cerkljansko hills, there are only one $5 \times 5 \mathrm{~km}$ UTM square with records after year 1989 (i.e. in the eastern part of the Julian Alps) and nine $5 \times 5 \mathrm{~km}$ UTM squares with presence of the species before year 1990. My records from surroundings of Bovec (UM83/2), Banjška planota (UL99/3, VL09/1, UM90/4) and Kanalski Kolovrat (UM91/4) show that in this part of NW Slovenia the species was also found in the last decade (Fig. 4).

\section{Phengaris arion (Linnaeus, 1758)}

(I) - VL67/2: 8.8.1994, Notranjska, Bloška planota, Berinjek, 730 m, 460888/72796; WM12/ 1: 3.8.2001, Štajerska, Ponikovska planota, Marof, $410 \mathrm{~m}, 512776 / 129825$.

(II) - VM11/4: 17.7.2006, Primorska, Baška grapa, Obloke, 477 m, 416282/115279.

(iv) - New information on species occurrence in the hilly region between Kamniško-Savinjske Alps and Dravinja hills: the species distribution map in the Atlas (Verovnik et al. 2012: pp. 225) indicates that there is only one locality in this region before year 1990. My record from Ponikovska plataeu (WM12/1) shows that the species occurred in the hilly region between the Kamniško-Savinjske Alps and the Dravinja hills at least 10 years ago.

Phengaris teleius (Bergsträsser, 1779)

(II) - VL68/1: 16.7.2008, Dolenjska, Ljubljansko barje, Ig, 290 m, 464960/91030. (iv) - New information on the vertical distribution of species: according to the data in the Atlas (Verovnik et al. 2012: pp. 226), the species is present on Mt. Nanos at heights up to $800 \mathrm{~m}$ a. s. 1. My record from Mt. Nanos shows that species occurs there also at higher altitudes, i.e. within the middle montane zone: VL27/4: 22.8.2004, Primorska, Nanos, Laniški vrh, 1040 m, 425555/71520.

Phengaris alcon (Denis \& Schiffermüller, 1775)

(I) - VM53/2: 21.7.2010, Kamniško-Savinjske Alpe, Storžič, Psica, 1612 m, 453730/133838.

(ii) - The presence of species in areas which in the Atlas are designated as "white spots": western part of Kamniško-Savinjske Alps (P. alcon f. rebeli).

(iv) - New information on the vertical distribution of species: according to the data in the Atlas (Verovnik et al. 2012: pp. 230), the species occurs in the Kamniško-Savinjske Alps at heights up to $1500 \mathrm{~m}$ a. s. 1. My record from Mt. Storžič (VM53/2) shows that species is also present at altitudes above $1600 \mathrm{~m}$.

\section{Plebeius argus (Linnaeus, 1758)}

(II) - UL99/3: 9.6.2006, Primorska, Banjšice, Visoko, 713 m, 399375/98918; 22.6.2006, Primorska, Banjšice, Tlaka, 710 m, 399926/98970; 9.6.2006, Primorska, Banjšice, Pičulini, 703 m, 399772/99053; Kuk, 760 m, 400311/99855; UM83/ 2: 14.6.2006, Primorska, Bovec, Podčela, $341 \mathrm{~m}$, 385026/131913; UM90/4: 30.6.2006, Primorska, Banjšice, Breščaki, 632 m, 398146/101314; UM 91/3: 14.6.2006, Primorska, Kobarid, Kamno, 173 m, 395606/119343; VL16/4: 11.6.2003, Primorska, Kras, Brestovica pri Povirju, $405 \mathrm{~m}$, 418675/63611; 395 m, 418432/63811; VL46/2: 12.6.2003, Notranjska, Trnje (Pivka), Debeli vrh, $620 \mathrm{~m}, 442315 / 62512$; Sv. Trojica, $1040 \mathrm{~m}$, 443697/64811; VM10/1: 16.6.2006, Primorska, Dolenja Trebuša, Stopnik, 195 m, 413461/106663; WM86/2: 24.5.1995, Śtajerska, Hrastje-Mota, Vučja vas, 188 m, 584890/162842.

\section{Plebejus idas (Linnaeus, 1761)}

(I) - VL76/4: 22.6.2004, Dolenjska, Ribnica, Prigorica, $480 \mathrm{~m}$, 479834/63024.

(II) - VL16/2: 25.6.1997, Primorska, Sežana, Dol Leskovec, 300 m, 412212/62044; VL45/1: 9.7.2003, Notranjska, Pivška kotlina, Palčje, 580 m, 441922/59314; 545 m, 442850/60275; 540 m, $442967 / 60366$. 
(III) - VL26/3: 25.6.1997, Slovenija, Senožeče, Laže, 698 m, 428111/66702.

(ii) - The presence of species in areas which in the Atlas are designated as "white spots": central part of Kočevska region.

\section{Plebejus argyrognomon (Bergsträsser, 1779)}

(I) - VL78/1: 30.7.1995, Dolenjska, Grosuplje, Radensko polje, 326 m, 475287/86363.

\section{Plebejus orbitulus (de Prunner, 1798)}

(iii) - New information on locality of the species with very limited distribution in Slovenia: until 2005, only two areas of species distribution in Slovenia were known, i.e. the northwestern part (Mt. Mangart) and the northern part (Mt. Dolkova Špica, the Vršič pass) of the Julian Alps (Čelik et al. 2005). The overview of the distribution of recording in the period from 2000 to 2011 in the Atlas (Verovnik et al. 2012: pp. 35) indicates that the species was found in the new area, i.e. in the western part of the Julian Alps ("Mt. Kanin southern from Mangart", cf. Verovnik et al. 2012: pp. 240) in 2010. I observed the species in the western part of the Julian Alps already in 2006, at the locality northeastern from Mt. Kanin, i.e. UM83/1: 18.7.2006, Julijske Alpe, Prestreljenik, Lopa, 2150 m, 383667/137165.

\section{Aricia eumedon (Esper, 1780)}

(I) - UL99/3: 22.6.2006, Primorska, Banjšice, Sleme, 773 m, 399549/100511; VL18/1: 27.6.2002, Primorska, Trnovski gozd, Mala Gora, 1045 m, 411424/87178; 1175 m, 411708/87638.

(II) - VL46/2: 12.6.2003, Notranjska, Trnje (Pivka), Sv. Trojica, 1040 m, 443697/64811.

(i) - New areas of the species distribution: northern part of Primorska region (Banjška planota) (Fig. 5).

(iv) - New information on species occurrence in Trnovski gozd region: according to the species distribution map in the Atlas (Verovnik et al. 2012:

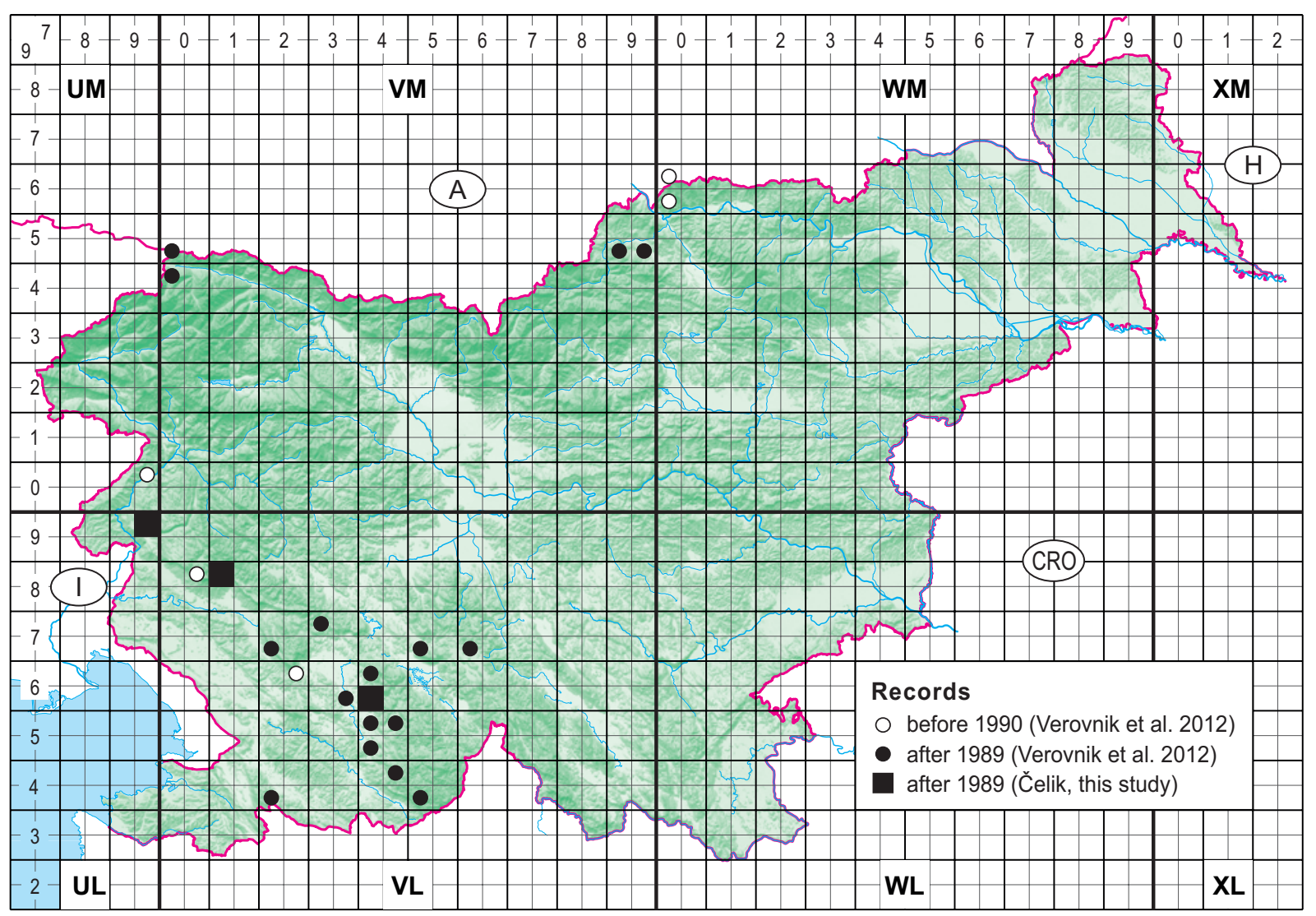

Figure 5: New records on the distribution of Aricia eumedon (Esper, 1780) (Čelik, this study) according to the Atlas of butterflies (Lepidoptera: Rhopalocera) of Slovenia (Verovnik et al. 2012: pp. 243).

Slika 5: Novi podatki o razširjenosti vrste Aricia eumedon (Esper, 1780) (Čelik, ta študija) glede na podatke v Atlasu dnevnih metuljev (Lepidoptera: Rhopalocera) Slovenije (Verovnik s sod. 2012: str. 243). 
pp. 243), the species was found in that region only before 1990 , only in one $5 \times 5 \mathrm{~km}$ UTM square (VL08/3). My record from Mala Gora (VL18/1) shows that in Trnovski gozd region the species was also observed in the last decade (Fig. 5).

\section{Aricia agestis (Denis \& Schiffermüller, 1775)}

(I) - UM83/1: 18.7.2006, Julijske Alpe, Bovec, Lopa, $2146 \mathrm{~m}, 383667 / 137165$; WM12/1: 21.7.2002, Stajerska, Ponikovska planota, Marof, $410 \mathrm{~m}, 512776 / 129825$.

(II) - VL06/1: 5.7.2002, Primorska, Kras, Pliskovica, $220 \mathrm{~m}$, 404742/70251; Reber, $230 \mathrm{~m}$, 404414/70455; Vrhek, 290 m, 406303/70780; VL 59/2: 23.5.1995, Notranjska, Ljubljansko barje, Bevke, 286 m, 451729/93527; VL87/4: 9.6.2004, Dolenjska, Suha krajina, Brezovi Dol, $390 \mathrm{~m}$, 489089/75096; Primča vas, 360 m, 486197/75503; VL97/2: 9.6.2004, Dolenjska, Suha krajina, Veliko Lipje, $310 \mathrm{~m}, 493787 / 73768 ;$ VM71/2: 22.8.2003, Gorenjska, Radomlje, Rova, 337 m, 471304/114890; VM82/4: 21.7.2006, Gorenjska, Menina planina, Šavnica, 1415 m, 489305/122870.

(iv) - New information on the vertical distribution of species: according to the data in the Atlas (Verovnik et al. 2012: pp. 244), the highest confirmed record of the species is from the Julian Alps at an altitude of around 1800 meters. My record from Mt. Lopa (UM83/1) shows that species occurs on south-facing slopes in the western part of the Julian Alps also at altitudes above $2100 \mathrm{~m}$.

\section{Aricia artaxerxes (Fabricius, 1793)}

(II) - VM53/2: 21.7.2010, Kamniško-Savinjske Alpe, Storžič, $1625 \mathrm{~m}, 453907 / 133783 ; 1770 \mathrm{~m}$, 454119/133873; $1895 \mathrm{~m}, 454302 / 133963 ; 2050 \mathrm{~m}$, 454491/134010; Pl. Javornik, 1574 m, 453255/ 133837; Psica, $1612 \mathrm{~m}, 453730 / 133838 ; 1570 \mathrm{~m}$, $453448 / 133846$.

(iv) - New information on species occurrence in western part of Kamniško-Savinjske Alps: according to the data in the Atlas (Verovnik et al. 2012: pp. 247), the species was observed in that region only before 1990. My records from Mt. Storžič (VM53/2) show that species is still present in the western part of the Kamniško-Savinjske Alps.

\section{Cyaniris semiargus (Rottemburg, 1775)}

(I) - WL26/1: 28.6.1994, Dolenjska, Gorjanci, Sv. Miklavž, 941 m, 525182/69956; WM12/1:
31.5.1987, Štajerska, Ponikovska planota, Marof, $410 \mathrm{~m}, 512776 / 129825$.

(II) - UM83/1: 18.7.2006, Julijske Alpe, Bovec, Lopa, 2036 m, 384480/137000; UM83/3: 18.7.2006, Primorska, Julijske Alpe, Bovec, Črnelski Vršiči, 2017 m, 386750/137840; Goričica, 2115 m, 386560/137970; UM90/4: 30.6.2006, Primorska, Banjšice, Breščaki, 632 m, 398146/101314; VL16/4: 11.6.2003, Primorska, Kras, Brestovica pri Povirju, $420 \mathrm{~m}, 419143 / 63698 ; 400 \mathrm{~m}$, 418317/63885; VL87/3: 9.6.2004, Dolenjska, Fužina, Grintovec, 310 m, 487004/78823; VM82/4: 21.7.2006, Gorenjska, Menina planina, Šavnica, $1422 \mathrm{~m}, 489550 / 122680 ; 1415 \mathrm{~m}, 489305 / 122870$; 1376 m, 488888/123100; Dom na Menini planini, $1468 \mathrm{~m}, 486312 / 123800$; Vivodnik, $1423 \mathrm{~m}$, $485860 / 124290$.

(iv) - New information on species occurrence in Dolenjska region: according to the data in the Atlas (Verovnik et al. 2012: pp. 249), the species was observed in the eastern part of Dolenjska region (i.e. surroundings of Novo mesto, eastern part of Gorjanci Mountains) only before year 1990. My record from St. Miklavž (WL26/1) shows that species occurred in that part of Dolenjska region also after year 1990.

\section{Polyommatus dorylas (Denis \& Schiffermüller, 1775)}

(II) - UL99/3: 12.7.2006, 22.6.2006, Primorska, Banjšice, Visoko, 755 m, 399727/98814; 22.9 . 2006, Primorska, Banjšice, Visoko, 713 m, 399375/ 98918; 12.7.2006, Primorska, Banjšice, Kuk, 760 m, 400311/99855; 12.7.2006, 9.6.2006, Primorska, Banjšice, Sleme, 769 m, 399542/100466; VL09/1: 12.7.2006, Primorska, Banjšice, Sveto, $762 \mathrm{~m}$, 400355/100030; VL15/1: 11.6.2006, Primorska, Lokev, Jirmanec, $658 \mathrm{~m}, 414621 / 56055$; VL47/4: 27.7.1994, Notranjska, Cerknica, Kamna Gorica, 620 m, 450339/73257; WL26/1: 28.6.1994, Dolenjska, Gorjanci, Sv. Miklavž, 941 m, 525182/69956.

(ii) - The presence of species in areas which in the Atlas are designated as "white spots": Banjška planota.

\section{Polyommatus amandus (Schneider, 1792)}

(I) - UL99/3: 22.6.2006, Primorska, Banjšice, Sleme, 773 m, 399549/100511; UM91/4: 16.6.2006, Primorska, Kanalski Kolovrat, Avško, 655 m, 397757/111482; WL03/1: 1.6.2004, Dolenjska, Predgrad, Dol, 240 m, 504849/38914. 


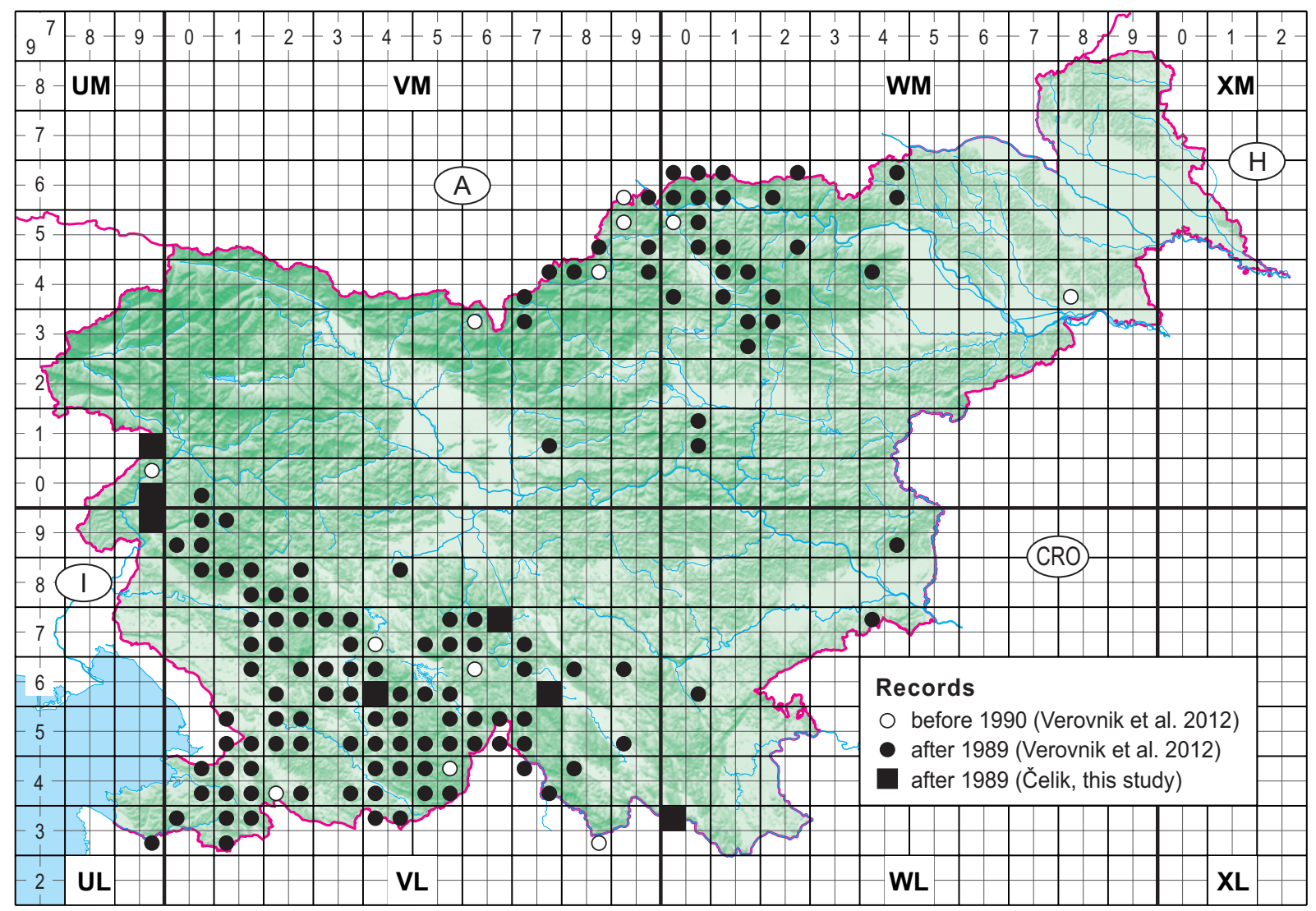

Figure 6: New records on the distribution of Polyommatus amandus (Schneider, 1792) (Čelik, this study) according to the Atlas of butterflies (Lepidoptera: Rhopalocera) of Slovenia (Verovnik et al. 2012: pp. 255).

Slika 6: Novi podatki o razširjenosti vrste Polyommatus amandus (Schneider, 1792) (Čelik, ta študija) glede na podatke v Atlasu dnevnih metuljev (Lepidoptera: Rhopalocera) Slovenije (Verovnik s sod. 2012: str. 255).

(II) - UM90/4: 30.6.2006, Primorska, Kanal, Kanalski Vrh, 350 m, 395765/104840; VL46/2: 12.6.2003, Notranjska, Trnje (Pivka), Sovič, 750 m, 445042/64107; VL67/3: 15.6.2004, Dolenjska, Rašica, Knej, 480 m, 469860/78278; VL76/4: 22.6.2004, Dolenjska, Ribnica, Ugar, 490 m, $478824 / 64153$.

(i) - New areas and range limits of the species distribution: my records from Posočje region (UL99/3, UM90/4, UM91/4) and Kolpa river valley (WL03/1) show that species range in Slovenia extends to the Italian border in the northwest, and to Bela Krajina region in the southeast (Fig. 6).

\section{Polyommatus thersites (Cantener, 1835)}

(I) - VL16/4: 11.6.2003, Primorska, Kras, Brestovica pri Povirju, 405 m, 418675/63611; VL47/1: 12.8.1993, Notranjska, Planinsko polje, Zeleno vrje, $450 \mathrm{~m}, 441885 / 78750$.
(II) - VL06/3: 5.7.2002, Primorska, Pliskovica, Vrhek, 290 m, 406303/70780.

(ii) - The presence of species in areas which in the Atlas are designated as "white spots": Kras, central part of Notranjska region.

\section{Polyommatus icarus (Rottemburg, 1775)}

(II) - UM90/4: 30.6.2006, Primorska, Banjšice, Breščaki, 632 m, 398146/101314; 18.5.2006, Primorska, Banjšice, Lužarji, 697 m, 399115/103927; UM91/4: 16.6.2006, Primorska, Kanalski Kolovrat, Avško, 655 m, 397757/111482; VM10/1: 16.6.2006, Primorska, Dolenja Trebuša, Stopnik, $195 \mathrm{~m}$, 413461/106663.

(III) - VL16/2: 9.6.1995, Primorska, Sežana, Dol Leskovec, 302 m, 412228/62044.

\section{Polyommatus coridon (Poda, 1761)}

(I) - WM12/1: 21.7.2002， 15.8.1988, Štajer- 
ska, Ponikovska planota, Marof, 410 m, 512776/ 129825.

(II) - UL99/3: 5.9.2006, 23.8.2006, Primorska, Banjšice, Visoko, 755 m, 399727/98814; 22.9.2006, 5.9.2006, 23.8.2006, Primorska, Banjšice, Visoko, 713 m, 399375/98918; 23.8.2006, Primorska, Banjšice, Tlaka, 705 m, 399466/99091; $702 \mathrm{~m}$, 399593/99162; 5.9.2006, 23.8.2006, Primorska, Banjšice, Kuk, 760 m, 400311/99855; 5.9.2006, 23.8.2006, Primorska, Banjšice, Sleme, 769 m, 399542/100466; 773 m, 399549/100511; VL47/4: 27.7.1994, Notranjska, Cerknica, Kamna Gorica, 620 m, 450339/73257; VM32/2: 17.7.2005, Gorenjska, Jelovica, Dražgoše, 800 m, 435206/123986.

(III) - VM32/1: 17.7.2005, Gorenjska, Jelovica, Spodnja Lipnica, 1001 m, 433570/130640.

(ii) - The presence of species in areas which in the Atlas are designated as "white spots": Banjška planota.

\section{Libythea celtis (Laicharting, 1782)}

(I) - VL93/1: 6.7.2004, Dolenjska, dolina reke Kolpe, Sapnik, 210 m, 493323/38064.

(II) - VL06/1: 11.6.2003, Primorska, Kras, Pliskovica, 220 m, 404645/70288; VL16/4: 11.6. 2003, Primorska, Kras, Brestovica pri Povirju, 400 m, 418317/63885; VL19/1: 20.6.2007, Primorska, Vojsko, Gačnik, 923 m, 413750/100070; VL44/2: 21.4.2004, Primorska, Ilirska Bistrica, Vrbica, 420 m, 444231/44990; VL94/2: 6.7.2004, Dolenjska, Podstene pri Kostelu, Dolenji Potok, 300 m, 491880/41854; Gorenji Potok, 335 m, $490680 / 42174$.

(III) - VL06/3: 5.4.1995, Primorska, Kras, Pliskovica, $280 \mathrm{~m}, 405619 / 70714$; VL16/2: 5.4 . 1995, Primorska, Sežana, Dol Leskovec, 310 m, 412167/62026.

(i) - New area of the species distribution: Kolpa river valley. It represents the easternmost locality (VL94/2) of the species distribution in Slovenia.

\section{Argynnis paphia (Linnaeus, 1758)}

(I) - VL99/3: 11.7.2003, Dolenjska, Polšnik, Golek, 680 m, 498250/101025.

(II) - UM90/1: 30.6.2006, Primorska, Kanalski Kolovrat, Britof, 264 m, 391104/107092; UM90/4: 30.6.2006, Primorska, Kanal, Kanalski Vrh, 470 m, 395800/103890; 350 m, 395765/104840; VL16/2: 25.6.1997, Primorska, Sežana, Dol Leskovec, 300 m, 412212/62044; VL19/1: 17.7.2006, Pri- morska, Vojsko, Gačnik, 556 m, 412915/100595; VL68/3: 3.7.2009, Dolenjska, Pijava Gorica, Rogovila, 295 m, 466680/88550; VM40/3: 5.7.2004, Gorenjska, Škofja Loka, Dolinski mlin, 430 m, 447100/108928; VM53/2: 21.7.2010, KamniškoSavinjske Alpe, Storžič, Pl. Velika Poljana, 1405 m, 452500/133856; VM94/2: 18.7.2005, Štajerska, Bele Vode, Senovršnik, 940 m, 495278/142158; VM94/4: 18.7.2005, Štajerska, Zavodnje, Mazetov graben, $555 \mathrm{~m}, 500250 / 141326$; Vovke $590 \mathrm{~m}$, 499460/141500; 18.7.2005, Štajerska, Bele Vode, Srnenski vrh, $952 \mathrm{~m}, 495894 / 141672$; Andrejev dom na Slemenu, 1087 m, 496380/143168; WM03/ 1: 18.7.2005, Štajerska, Šoštanj, Kurja vas, 408 m, 500692/138400.

(III) - VM32/4: 17.7.2005, Gorenjska, Jelovica, Dražgoše, 1020 m, 438000/123300.

\section{Argynnis aglaja (Linnaeus, 1758)}

(I) - VL06/3: 5.7.2002, Primorska, Kras, Dutovlje, 275 m, 408528/69110; WM03/1: 18.7 . 2005, Štajerska, Šoštanj, Kurja vas, 408 m, 500692/138400.

(II) - UM83/3: 18.7.2006, Julijske Alpe, Bovec, Črnelski Vršiči, 2017 m, 386750/137840; UM90/1: 30.6.2006, Primorska, Kanalski Kolovrat, Lig, 607 m, 392810/107048; VL23/2: 21.6. 2002, Primorska, Rakitovec, Ubred, $660 \mathrm{~m}$, 421031/36003; VL46/2: 12.6.2003, Notranjska, Trnje (Pivka), Debeli vrh, 620 m, 442315/62512; VM11/4: 17.7.2006, Primorska, Baška grapa, Zakojca, 639 m, 418318/114023; Obloke, $464 \mathrm{~m}$, 416304/115252; 558 m, 416154/115482; VM12/2: 25.7.2005, Julijske Alpe, Rodica, Pl. Suha, 1388 m, 412041/122509; Javorje, 1115 m, 412850/123250; VM94/2: 18.7.2005, Štajerska, Bele Vode, Senovršnik, $940 \mathrm{~m}, 495278 / 142158$.

(III) - VM94/4: 18.7.2005, Štajerska, Zavodnje, Vovke, $590 \mathrm{~m}$, 499460/141500; Srnenski vrh, 952 m, 495894/141672; Šentvid pri Zavodnju, 934 m, 498634/143192; 1000 m, 498398/143348; 18.7.2005, Štajerska, Bele Vode, Andrejev dom na Slemenu, 1087 m, 496380/143168.

(iv) - New information on the vertical distribution of species: according to the data in the Atlas (Verovnik et al. 2012: pp. 272), the species is present at heights up to $1900 \mathrm{~m}$ a. s. l. only in the Julian Alps. My record from Mt. Črnelski Vršiči (UM83/3) shows that on the south-facing slopes in the western part of the Julian Alps the species occurs also above 2000 meters. 
Argynnis adippe (Denis \& Schiffermüller, 1775)

(I) - VL35/2: 5.8.1994, Primorska, Dolnje Vreme, Ribnica, 369 m, 432202/55143; VL99/3: 11.7.2003, Dolenjska, Polšnik, Golek, $690 \mathrm{~m}$, 497957/100781; VM41/2: 5.7.2004, Gorenjska, Škofja Loka, Zminec, $390 \mathrm{~m}, 443306 / 111785$; WL07/4: 29.6.1994, Dolenjska, Novo mesto, Zalog, 163 m, 509502/72582; WM23/2: 29.6.2005, Štajerska, Lipa pri Frankolovem, Selce, 633 m, 523152/134338.

(II) - VL15/1: 11.6.2006, Primorska, Lokev, Jirmanec, $611 \mathrm{~m}, 414279 / 56420$; 25.6.1997, Primorska, Sežana, Mlave, 400 m, 414734/59704; VL16/2: 25.6.1997, Primorska, Sežana, Dol Leskovec, 300 m, 412212/62044; Dane pri Sežani, Koblak, 350 m, 414584/64790; Filipčje Brdo, Prjunščine, $350 \mathrm{~m}$, 414702/65614.

(III) - VL56/1: 23.6.1994, Notranjska, Cerkniško polje, Martinjak, $551 \mathrm{~m}, 454051 / 69189$; VM11/4: 17.7.2006, Primorska, Baška grapa, Zakojca, 639 m, 418318/114023.

(ii) - The presence of species in areas which in the Atlas are designated as "white spots": Posavsko hills, Konjiško hills, area around Novo mesto.

\section{Argynnis niobe (Linnaeus, 1758)}

(I) - VL23/2: 21.6.2002, Primorska, Rakitovec, Ubred, $660 \mathrm{~m}, 421031 / 36003$; VL47/1: 2.7.1993, Notranjska, Planinsko polje, Gmajna, 442 m, 443045/78418; 8.7.1993, Notranjska, Planinsko polje, Škofji lom, $442 \mathrm{~m}, 442211 / 80853$; WL26/1: 28.6.1994, Dolenjska, Gorjanci, Sv. Miklavž, $941 \mathrm{~m}$, 525182/69956.

(II) - UL99/3: 9.6.2006, Primorska, Grgar, Grašišče, 312 m, 397513/96420; 22.6.2006, Primorska, Grgar, Zabrdo, 466 m, 398089/98029; Banjšice, Pičulini, $710 \mathrm{~m}$, 399926/98970; 12.7.2006, Primorska, Banjšice, Kuk, 760 m, 400311/99855; VL15/1: 11.6.2006, Primorska, Lokev, Jirmanec, 421 m, 413383/56889; VL16/1: 11.6.2003, Primorska, Kras, Tomaj, 340 m, 411103/68920; VL16/4: 11.6.2003, Primorska, Kras, Brestovica pri Povirju, 400 m, 418317/63885; VM53/2: 21.7.2010, Kamniško-Savinjske Alpe, Storžič, Dom pod Storžičem, 1122 m, 453485/135043; VM73/4: 21.7.2009, Kamniško-Savinjske Alpe, Dleskovška planota, Dleskovec, 1830 m, 476423/134110; VM94/2: 18.7. 2005, Štajerska, Bele Vode, Senovršnik, 940 m, $495278 / 142158$.

(III) - VM93/3: 18.7.2005, Štajerska, Bele Vode, Razpadovnik, 611 m, 498052/140150. (ii) - The presence of species in areas which in the Atlas are designated as "white spots": Banjška planota, central part of Notranjska region, eastern part of Dolenjska region.

(iv) - New information on the vertical distribution of species: according to the data in the Atlas (Verovnik et al. 2012: pp. 276), the species is present in the Alps at heights up to $1700 \mathrm{~m}$ a. s. 1 . My record from Dleskovška planota (VM73/4) shows that species occurs also at altitudes above 1800 meters.

\section{Issoria lathonia (Linnaeus, 1758)}

(II) - VL09/1: 12.7.2006, Primorska, Banjšice, Sveto, 762 m, 400355/100030; VL27/4: 22.8. 2004, Primorska, Nanos, Laniški vrh, 1040 m, $425555 / 71520$.

(III) - VL16/2: 13.4.1995, Primorska, Sežana, Dol Leskovec, 305 m, 412246/62030.

(ii) - The presence of species in areas which in the Atlas are designated as "white spots": Banjška planota.

\section{Brenthis ino (Rottemburg, 1775)}

(I) - UL99/3: 12.7.2006, 22.6.2006, Primorska, Banjšice, Visoko, 755 m, 399727/98814; 12.7.2006, 22.6.2006, Primorska, Banjšice, Visoko, $713 \mathrm{~m}$, 399375/98918; 22.6.2006, Primorska, Banjšice, Pičulini, $710 \mathrm{~m}, 399926 / 98970 ; 12.7 .2006,22.6 .2006$, Primorska, Banjšice, Kuk, 760 m, 400311/99855; UM83/2: 14.6.2006, Primorska, Bovec, Podčela, 341 m, 385026/131913; UM91/4: 16.6.2006, Primorska, Doblar, Lepenka, 344 m, 399024/111763; VL19/1: 20.6.2007, Primorska, Vojsko, Gačnik, 907 m, 413050/100450; 910 m, 412894/100598; 17.7.2006, Primorska, Gorenja Trebuša, Makčeva grapa, 556 m, 410707/100760; VL93/1: 6.7.2004, Dolenjska, dolina Kolpe, Slavski Laz, 210 m, 492234/36930.

(II) - VL76/4: 22.6.2004, Dolenjska, Ribnica, Zalužje, 480 m, 479834/63024; Ugar, 490 m, 478824/64153; 480 m, 478571/64240; VL83/4: 6.7. 2004, Dolenjska, Petrina, Grivački jarek, $310 \mathrm{~m}$, 488080/35827; VL84/4: 6.7.2004, Dolenjska, Morava, Suhor, $530 \mathrm{~m}, 490041 / 43757$.

(i) - New areas of the species distribution: the lower part of Soča river valley, Banjška planota, Idrijsko hills (Fig. 7). 


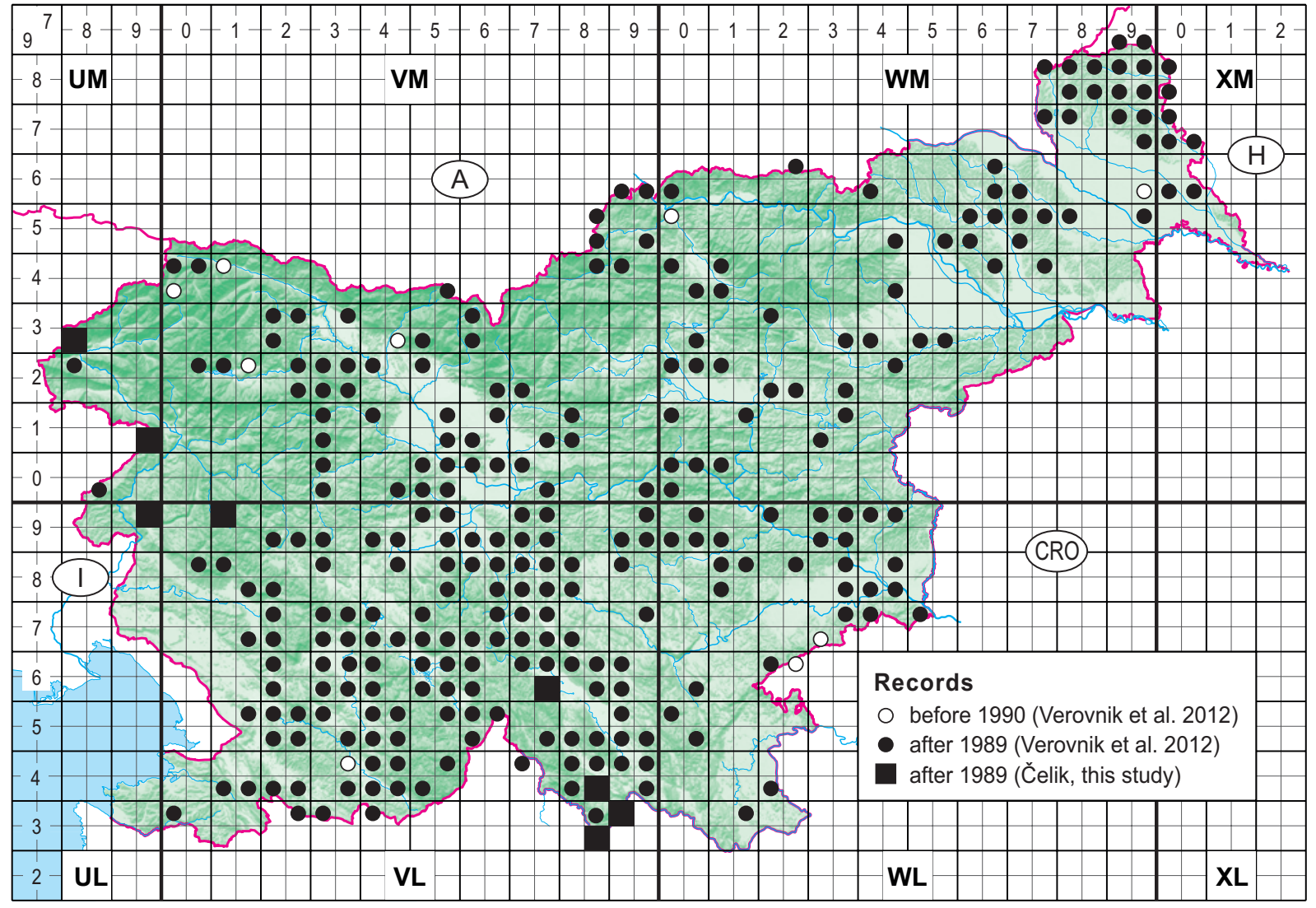

Figure 7: New records on the distribution of Brenthis ino (Rottemburg, 1775) (Schneider, 1792) (Čelik, this study) according to the Atlas of butterflies (Lepidoptera: Rhopalocera) of Slovenia (Verovnik et al. 2012: pp. 281).

Slika 7: Novi podatki o razširjenosti vrste Brenthis ino (Rottemburg, 1775) (Schneider, 1792) (Čelik, ta študija) glede na podatke v Atlasu dnevnih metuljev (Lepidoptera: Rhopalocera) Slovenije (Verovnik s sod. 2012: str. 281).

\section{Brenthis daphne (Bergsträsser, 1780)}

(I) - WL26/1: 28.6.1994: Dolenjska, Gorjanci, Sv. Miklavž, $941 \mathrm{~m}, 525182 / 69956$.

(II) - UM83/2: 14.6.2006, Primorska, Bovec, Podčela, 343 m, 384910/131553; UM90/4: 30.6. 2006, Primorska, Banjšice, Breščaki, $632 \mathrm{~m}$, 398146/101314; UM91/3: 14.6.2006, Primorska, Kobarid, Kamno, 170 m, 396080/119044; VL09/1: 12.7.2006, Primorska, Banjšice, Sveto, $762 \mathrm{~m}$, 400355/100030; VL15/1: 11.6.2006, Primorska, Lokev, Jirmanec, 421 m, 413383/56889; VL46/2: 12.6.2003, Notranjska, Trnje (Pivka), Debeli vrh, 620 m, 442315/62512; VL84/3: 6.7.2004, Dolenjska, Kočevska Reka, Novi Lazi, 525 m, 487702/47269; VM40/3: 5.7.2004, Gorenjska, Škofja Loka, Hrastnica, 500 m, 446728/107803; $430 \mathrm{~m}, 447100 / 108928 ; 380 \mathrm{~m}, 447303 / 111248$; WL17/1: 18.5.2004, Dolenjska, Novo mesto, Sela pri Štravberku, 510 m, 515023/79451; WM22/1: 29.6.2005, Štajerska, Frankolovo, Rakova Steza, $330 \mathrm{~m}, 524113 / 130770$.
(III) - UM90/3: 16.6.2006, Primorska, Doblar, Doblarec, 130 m, 398812/108738; VM11/4: 17.7.2006, Primorska, Baška grapa, Zakojca, 639 m, 418318/114023; 612 m, 417675/114396; Hudajužna, 424 m, 416263/114968; Obloke, 477 m, 416282/115279; 526 m, 416241/115388; VM94/2: 18.7.2005, Štajerska, Bele Vode, Senovršnik, $940 \mathrm{~m}, 495278 / 142158$.

Brenthis hecate (Denis \& Schiffermüller, 1775)

(I) - VL48/2: 8.7.1993, Notranjska, Planinsko polje, Strževce, $441 \mathrm{~m}, 441884 / 81049$.

(II) - UM90/4: 30.6.2006, Primorska, Banjšice, Breščaki， $632 \mathrm{~m}$, 398146/101314; VL16/1: 11.6.2003, Primorska, Kras, Tomaj, 340 m, 411137/ 68931; VL16/4: 11.6.2003, Primorska, Kras, Brestovica pri Povirju, $395 \mathrm{~m}, 418432 / 63811$; VL47/1: 18.7.1994, Notranjska, Planinsko polje, Trzne, 447 m, 443296/78402; 2.7.1993, Notranjska, Planinsko polje, Luže, 444 m, 443658/78765; 16.6.1993, Notranjska, Planinsko polje, Log, 
446 m, 441916/79372; 1.7.1993, Notranjska, Planinsko polje, Kosovnica, $445 \mathrm{~m}, 440737 / 80190$; 8.7.1993, Notranjska, Planinsko polje, Škofji lom, 442 m, 442211/80853; VM33/1: 23.7.1996, Gorenjska, Bled, Koritno, 450 m, 433552/136357.

(III) - VL16/2: 25.6.1997, Primorska, Sežana, Dol Leskovec, 300 m, 412212/62044; Filipčje Brdo, Prjunščine, 350 m, 414702/65614.

(ii) - The presence of species in areas which in the Atlas are designated as "white spots": central part of Notranjska region.

\section{Boloria euphrosyne (Linnaeus, 1758)}

(I) - UM91/3: 14.6.2006, Primorska, Kolovrat, Foni, 569 m, 396901/117862; VL66/1: 16.5. 1994, Notranjska, Bloška planota, Resja, 716 m, 462212/67889; WM12/1: 4.6.1988, Štajerska, Ponikovska planota, Marof, $410 \mathrm{~m}, 512776 / 129825$.

(II) - VL15/1: 11.5.2008, Primorska, Sežana, Lipica, 378 m, 412860/58600;369 m,412960/59140; 379 m, 412840/59320; VL84/1: 19.5.2011, Dolenjska, Borovec pri Kočevski Reki, Dragarji, 868 m, 480803/46557; VM10/1: 16.6.2006, Primorska, Dolenja Trebuša, Stopnik, 195 m, 413461/106663; WM88/4: 11.6.2004, Prekmurje, Goričko, Mačkovci, $340 \mathrm{~m}, 587633 / 183486$.

(III) - UL97/1: 5.4.1995, Primorska, Kras, Sela na Krasu, 230 m, 393076/76043.

(i) - New area of the species distribution: southern edge of the Julian Alps (Kolovrat). It represents the western limit of the species distribution in Slovenia.

(iv) - New information on species occurrence in Goričko region: according to the data in the Atlas (Verovnik et al. 2012: pp. 287), the species was found only in the southeastern and northern (one $5 \times 5 \mathrm{~km}$ UTM square, i.e. WM88/3; with presence of species only before 1990) part of the region despite of intensive field surveys of butterfly fauna in Goričko region in years 2010 and 2011 (cf. Verovnik et al. 2012: pp. 35, 44-46). My record from the surroundings of Mačkovci (WM88/4) shows that the species occurred in the northern part of Goričko region at least until year 2004.

\section{Boloria selene (Denis \& Schiffermüller, 1775)}

(I) - VL36/3: 14.7.2004, Notranjska, Postojna, Veliki Otok, $517 \mathrm{~m}, 438175 / 70722 ; 520 \mathrm{~m}$, 437946/70733; VL47/1: 27.5.2010, Notranjska, Planinsko polje, Liplje, $447 \mathrm{~m}, 442615 / 78360$; 18.7.1994, Notranjska, Planinsko polje, Trzne,
447 m, 443296/78402; VL76/4: 22.6.2004, Dolenjska, Ribnica, Ugar, 490 m, 478824/64153; VL77/1: 15.6.2004, Dolenjska, Rašica, Podlog, $480 \mathrm{~m}, 470524 / 78676$.

(i) - New areas of the species distribution: Notranjska region, Kočevska region (Fig. 8). My records from those regions show that species range in Slovenia still extends at least 20 kilometers further to the south and southwest (cf. Čelik \& Rebeušek 1996: pp. 69) than it is presented on the species distribution map in the Atlas (cf. Verovnik et al. 2012: pp. 291).

\section{Boloria dia (Linnaeus, 1767)}

(I) - VL19/1: 20.6.2007, Primorska, Vojsko, Gačnik, 909 m, 413050/100480.

(II) - UL99/3: 5.9.2006, Primorska, Banjšice, Sleme, 769 m, 399542/100466; UM90/1:30.6.2006, Primorska, Kanalski Kolovrat, Čolnica, 489 m, 393760/106520; UM90/4: 30.6.2006, Primorska, Banjšice, Breščaki, 632 m, 398146/101314; VL09/ 1: 12.7.2006, Primorska, Banjšice, Sveto, $762 \mathrm{~m}$, 400355/100030; VL26/2: 13.5.2005, Primorska, Senožeče, Škrljevica, 559 m, 423956/64300; VL56/2: 27.4.1994, Notranjska, Cerkniško polje, Otok, 568 m, 451970/65778; VL67/3: 15.6.2004, Dolenjska, Rašica, Knej, 480 m, 469860/78278; VM32/1: 17.7.2005, Gorenjska, Jelovica, Goška ravan, $1001 \mathrm{~m}, 433570 / 130640$; WL14/2: 19.5 . 2004, Bela Krajina, Grič pri Dobličah, Kralji, $320 \mathrm{~m}, 510572 / 46002$.

(ii) - The presence of species in areas which in the Atlas are designated as "white spots": Banjška planota, Idrijsko hills.

\section{Boloria pales (Denis \& Schiffermüller, 1775)}

(II) - UM83/3: 18.7.2006, Julijske Alpe, Bovec, Črnelski Vršiči, $1740 \mathrm{~m}, 387130 / 136950$; $1780 \mathrm{~m}, 387060 / 137280 ; 2017 \mathrm{~m}, 386750 / 137840$; $2157 \mathrm{~m}, 386303 / 137855 ; 2115 \mathrm{~m}, 386560 / 137970$.

\section{Vanessa atalanta (Linnaeus, 1758)}

(I) - VL06/1: 29.5.2002, Primorska, Kras, Pliskovica, 230 m, 404633/70318; WL17/1: 11.5. 2004, Dolenjska, Novo mesto, Sela pri Štravberku, 510 m, 515059/79463; Nova Gora, 540 m, $514646 / 79674$.

(II) - UM82/3: 18.7.2003, Julijske Alpe, Drežniške Ravne, Veliki vrh, 1760 m, 390305/128860; UM90/1: 30.6.2006, Primorska, Kanalski Kolovrat, Lig, 607 m, 392810/107048; VL36/3: 11.8.2004, 


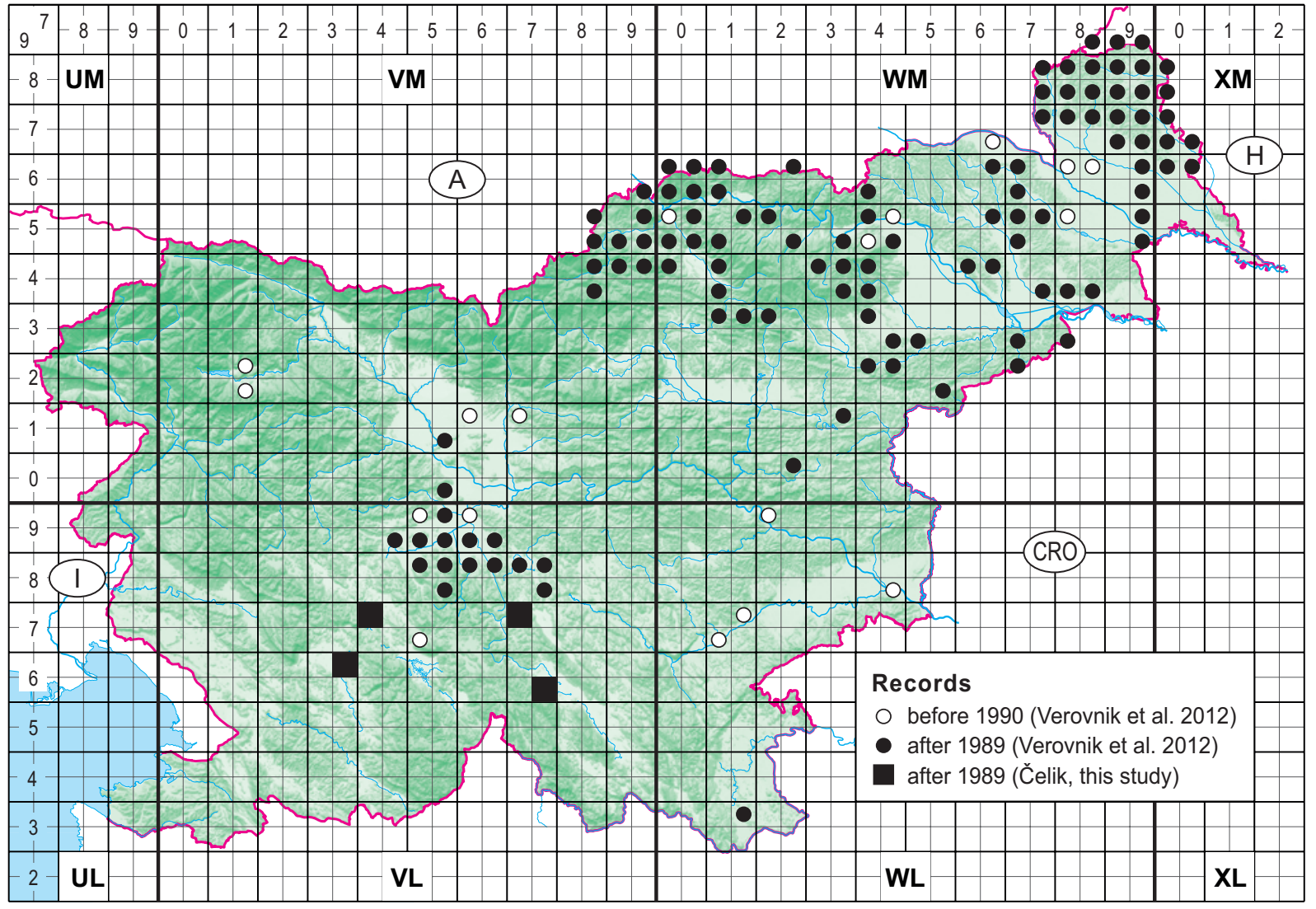

Figure 8: New records on the distribution of Boloria selene (Denis \& Schiffermüller, 1775) (Čelik, this study) according to the Atlas of butterflies (Lepidoptera: Rhopalocera) of Slovenia (Verovnik et al. 2012: pp. 291).

Slika 8: Novi podatki o razširjenosti vrste Boloria selene (Denis \& Schiffermüller, 1775) (Čelik, ta študija) glede na podatke v Atlasu dnevnih metuljev (Lepidoptera: Rhopalocera) Slovenije (Verovnik s sod. 2012: str. 291).

Notranjska, Postojna, Kremenca, 529 m, 438983/ 69115; VL47/1; 20.4.1993, Notranjska, Planinsko polje, Luže, $445 \mathrm{~m}, 443656 / 78740 ; 11.5 .1993$, Notranjska, Planinsko polje, Jakovski grič, $464 \mathrm{~m}$, 442830/79620; VL84/1: 6.7.2004, Dolenjska, Kočevska Reka, Mokri potok, 540 m, 485181/46829; VM73/2: 29.7.2008, Kamniško-Savinjske Alpe, Veliki Vrh, 1865 m, 475055/134380; 30.7.2008 Kamniško-SavinjskeAlpe, Belivrh, 1905 m,473930/134615; VM73/4: 21.7.2009, Kamniško-Savinjske Alpe, Dleskovška planota, Pl. Ravne, 1500 m, 477104/ 134000; 29.7.2008 Kamniško-Savinjske Alpe, Dleskovška planota, Smrekovec, $1580 \mathrm{~m}, 476865$ / 135005; WL03/1: 18.5.2011, Dolenjska, dolina reke Kolpe, Dol, 178 m, 503944/38501.

(III) - UL97/1: 26.9.2005, Primorska, Kras, Lokvica, 200 m, 390945/81035; VL34/3: 2.8.1994, Primorska, Ilirska Bistrica, Rečica, 400 m, 439830/ 47468; VL83/4: 6.7.2004, Dolenjska, Petrina, Grivački jarek, 310 m, 488080/35827; WM76/3: 23.8.1995, Štajerska, Mele, 201 m, 578556/169033. (ii) - The presence of species in areas which in the Atlas are designated as "white spots": hilly area on the right side of the lower part of Soča river valley (Kanalski Kolovrat), central part of Notranjska region.

\section{Vanessa cardui (Linnaeus, 1758)}

(I) - WM57/4: 12.6.1996， Štajerska， Sladki Vrh, $232 \mathrm{~m}$, 558564/172964.

(II) - UM82/3: 18.7.2003, Julijske Alpe, Drežniške Ravne, Veliki vrh, 1760 m, 390305/128860; UM83/1: 18.7.2006, Julijske Alpe, Bovec, Prevala, $2224 \mathrm{~m}, 383292 / 136837$; Lopa, $2036 \mathrm{~m}$, $384480 / 137000 ; 2146$ m, 383667/137165; Črnelski Vršiči, $1740 \mathrm{~m}, \quad 387130 / 136950 ; 2008 \mathrm{~m}$, 385600/137140; $1780 \mathrm{~m}, 387060 / 137280 ; 2028 \mathrm{~m}$, 386050/137430; $2017 \mathrm{~m}, 386750 / 137840 ; 2157 \mathrm{~m}$, $386303 / 137855 ; 2115 \mathrm{~m}, 386560 / 137970 ; 2320 \mathrm{~m}$, 386162/137990; VL15/1: 11.6.2006, Primorska, Lokev, Jirmanec, 658 m, 414621/56055; VL16/4: 11.6.2003, Primorska, Kras, Brestovica pri Po- 
virju, 405 m, 418675/63611; VL56/1: 23.6.1994, Notranjska, Cerkniško polje, Martinjak, $551 \mathrm{~m}$, 453769/69557; VL67/3: 22.6.2004, Dolenjska, Rašica, Knej, 480 m, 469946/78258; VL68/4: 5.6.2001, Dolenjska, Želimlje, Kamniti laz, 324 m, 468269/84267; VL77/1: 15.6.2004, Dolenjska, Rašica, Podlog, 480 m, 470524/78676; VM11/4: 17.7.2006, Primorska, Baška grapa, Zakojca, $639 \mathrm{~m}, \quad 418318 / 114023 ; 612 \mathrm{~m}, \quad 417675 / 114396$; Hudajužna, 424 m, 416263/114968; XM05/2: 25. 5.1995, Štajerska, Dolnja Bistrica, Kolišče Bobri, $168 \mathrm{~m}, 601024 / 154994$.

(III) - WM86/1: 11.6.2004, Prekmurje, Petanjci, Gornji Petanjci, 200 m, 581280/168268.

\section{Aglais io (Linnaeus, 1758)}

(I) - VL99/3: 11.7.2003, Dolenjska, Polšnik, Golek, 690 m, 497957/100781;WL06/4: 18.5.2004, Dolenjska, Dolenjske Toplice, Gorenje Sušice, $200 \mathrm{~m}, 506738 / 65617$; WM23/2: 29.6.2005, Štajerska, Frankolovo, Straža pri Dolu, $341 \mathrm{~m}$, $524180 / 132467$.

(II) - UL99/4: 30.6.2006, Primorska, Solkan, Sabotin, 96 m, 395920/93750; UM83/3: 18.7.2006, Julijske Alpe, Bovec, Črnelski Vršiči, 1740 m, 387130/136950; VL16/3: 26.9.2005, Primorska, Kras, Griže, 360 m, 418626/68350; $422 \mathrm{~m}$, 418441/68492; VL25/2: 14.4.2009, Notranjska, Brkini, Sušica, $409 \mathrm{~m}, 423047 / 55829 ; 407 \mathrm{~m}$, 422732/55882; 11.4.2009, Notranjska, Brkini, Sušica, 405 m, 422682/55905; 415 m, 423064/55933; 4.5.2009, Notranjska, Brkini, Sušica, 398 m, 422514/55969; VL34/4: 4.4.1994, Notranjska, Ilirska Bistrica, Harije, 484 m, 435695/45695; VL37/3: 23.4.1993, 4.5.1993, Notranjska, Planinsko polje, Sv. Jedert, 446 m, 440318/80177; 4.5.1993, Notranjska, Planinsko polje, Smukovci, 445 m, 439944/80732; 23.4.1993, Notranjska, Planinsko polje, Lanski vrh, $451 \mathrm{~m}, 439978 / 80923$; 4.5.1994, Notranjska, Planinsko polje, Lanski vrh, 451 m, 439855/81017; VM62/3: 15.7.2005, Kamniško-Savinjske Alpe, Kalški greben, Vrh Korena, 1857 m, 465696/131014; VM94/4: 18.7.2005, Štajerska, Zavodnje, Žlebnik, 713 m, 498950/141288; Mazetov graben, 555 m, 500250/141326; WL07/4: 29.6.1994, Dolenjska, Novo mesto, Zalog, $178 \mathrm{~m}$, 508548/72226; $163 \mathrm{~m}, 509502 / 72582$; WL14/1: 19.5.2004, Bela Krajina, Črnomelj, Mavrlen, $380 \mathrm{~m}, 510460 / 46285$.

(III) - VM73/2: 29.7.2008, Kamniško-Savinjske Alpe, Veliki Vrh, 1865 m, 475055/134380; 1770 m, 474240/134940; 30.7.2008, Kamniško-Savinjske Alpe, Ojstrica, 1950 m, 472420/134950;
2030 m, 472430/135180; 2050 m, 472410/135190; WM76/3: 23.8.1995, Štajerska, Mele, $201 \mathrm{~m}$, 578556/169033; WM77/2: 17.4.1996, Štajerska, Gornja Radgona, Podgrad, 209 m, 575214/171401; 20.4.1995, Stajerska, Lutverci, 212 m, 572088/ 172307.

(ii) - The presence of species in areas which in the Atlas are designated as "white spots": Posavsko hills, area around Novo mesto.

\section{Aglais urticae (Linnaeus, 1758)}

(I) - VL76/4: 22.6.2004, Dolenjska, Ribnica, Nemška vas, 490 m, 480174/64400; VL87/4: 9.6.2004, Dolenjska, Suha krajina, Ambrus, 360 m, 486197/75503; VL93/1: 6.7.2004, Dolenjska, dolina reke Kolpa, Slavski Lazi, 210 m, 492234/36930.

(II) - UM83/1: 18.7.2006, Julijske Alpe, Bovec, Prevala, $2224 \mathrm{~m}$, 383292/136837; Lopa, 2036 m, 384480/137000; Vršiči pod Lopo, 2146 m, 383667/137165; UM83/3: 18.7.2006, Julijske Alpe, Bovec, Črnelski Vršiči, 1740 m, 387130/136950; 1780 m, 387060/137280; 2028 m, 386050/137430; $2017 \mathrm{~m}, 386750 / 137840 ; 2115 \mathrm{~m}, 386560 / 137970$; Hudi Vršič, 2008 m, 385600/137140; Velika Črnelska špica, 2157 m, 386303/137855; UM92/1: 18.7.2003, Julijske Alpe, Drežniške Ravne, Krasji vrh, 1685 m, 391870/128236; VL15/1: 11.6.2006, Primorska, Lokev, Jirmanec, $658 \mathrm{~m}$, 414621/56055; VL16/3: 10.6.2005, Primorska, Kras, Griže, 360 m, 418626/68350; 23.4.2005, Primorska, Kras, Griže, $431 \mathrm{~m}, 418612 / 68468$; VL38/4: 29.5.1993, Notranjska, Planinsko polje, Lanski vrh, 452 m, 439658/81084; VL44/2: 21.4.2004, Primorska, Ilirska Bistrica, Koseze, 410 m, 440411/45504; VL67/4: 22.6.2004, Dolenjska, Rašica, Podstrmec, 560 m, 467439/72781; VM13/3: 17.7.2009, Julijske Alpe, Pokljuka, Klečica, 1862 m, 419150/139696; VM40/3: 5.7.2004, Gorenjska, Škofja Loka, Hrastnica, $400 \mathrm{~m}$, 447020/110330; VM51/2: 1.3.1995, Gorenjska, Sorško polje, Reteče, 354 m, 451902/113140; VM62/1: 15.7.2005, Kamniško-Savinjske Alpe, Ambrož pod Krvavcem, Veliki Zvoh, 1910 m, 464874/129340; 1953 m, 465167/129628; WL17/3: 11.5.2004, Dolenjska, Novo mesto, Srednje Grčevje, 480 m, 515938/79264; WM66/3: 20.4.1995, Štajerska, Gornja Radgona, Apače, 357 m, $569275 / 170625$.

(III) - VL88/2: 9.6.2004, Dolenjska, Ivančna Gorica, Krška vas, 280 m, 482588/82783; VM63/2: 16.7.2005, Kamniško-Savinjske Alpe, Kokrško se- 
dlo, Spodnje Jame, 1920 m, 465166/134016; Grintovec, Spodnji Dolci, 1850 m, 464340/134200; 1805 m, 464062/134294; WM77/2: 20.4.1995, Štajerska, Lutverci, 212 m, 572088/172307.

(ii) - The presence of species in areas which in the Atlas are designated as "white spots": western part of Dolenjska region, Kolpa river valley.

\section{Polygonia c-album (Linnaeus, 1758)}

(I) - VL06/1: 11.6.2003, Primorska, Kras, Pliskovica, Podbreg, 220 m, 404645/70288; WL06/4: 18.5.2004, Dolenjska, Dolenjske Toplice, Gorenje Sušice, $200 \mathrm{~m}$, 506738/65617.

(II) - UM91/4: 16.6.2006, Primorska, Doblar, Lepenka, 344 m, 399024/111763; VL15/1: 11.6.2006, Primorska, Lokev, Jirmanec, $611 \mathrm{~m}$, 414279/56420; VL16/1: 29.5.2002, Primorska, Kras, Tomaj, $380 \mathrm{~m}$, 410968/68755; VL16/4: 11.6.2003, Primorska, Kras, Brestovica pri Povirju, 400 m, 418317/63885; VL38/4: 29.5.1993, 18.5.1993, 4.5.1993, Notranjska, Planinsko polje, Lanski vrh, $452 \mathrm{~m}, 439658 / 81084$; VL48/2: 18.5.1993, Notranjska, Planinsko polje, Pod Stenami, 474 m, 441352/81210; VL56/4: 28.7.1994, Notranjska, Loška dolina, Dane, 580 m, 457070/62657; 26.7.1994, Notranjska, Loška dolina, Dane, 568 m, 456905/63091; VL83/4: 6.7.2004, Dolenjska, Petrina, Grivački jarek, 310 m, 488080/35827; VM40/1: 5.7.2004, Gorenjska, Zminec, Bodoljska grapa, 510 m, 444006/108598; VM73/2: 29.7.2008, Kamniško-Savinjske Alpe, Veliki Vrh, 1770 m, 474240/134940; WL17/1: 11.5.2004, Dolenjska, Novo mesto, Ždinja vas, $420 \mathrm{~m}, 513347 / 78293$; WM03/1: 18.7.2005, Štajerska, Šoštanj, Kurja vas, 408 m, 500692/138400; WM12/1: 19.4.1987, Štajerska, Ponikovska planota, Marof, $410 \mathrm{~m}$, 512776/129825; WM66/3: 20.4.1995, Štajerska, Gornja Radgona, Apače, 357 m, 569275/170625; WM76/2: 20.4.1995, Štajerska, Slovenske Gorice, Negova, 217 m, 574258/161598; XM05/2: 25.5.1995, Štajerska, Dolnja Bistrica, Kolišče Bobri, $168 \mathrm{~m}, 601024 / 154994$.

(III) - VL16/2: 8.4.1995, 5.4.1995, Primorska, Sežana, Dol Leskovec, 310 m, 412167/62026; VM50/1: 5.7.2004, Gorenjska, Sora, Ločnica, $400 \mathrm{~m}, 450528 / 108375 ; 370 \mathrm{~m}, 450950 / 109628$; WM77/2: 17.4.1996, Štajerska, Gornja Radgona, Podgrad, 209 m, 575214/171401.

(ii) - The presence of species in areas which in the Atlas are designated as "white spots": Kras, area around Novo mesto, Raduljsko hills.
Araschnia levana (Linnaeus, 1758)

(I) - VL38/4: 18.5.1993, 4.5.1993, Notranjska, Planinsko polje, Lanski vrh, 452 m, 439658/ 81084; VL47/1: 18.5.1993, Notranjska, Planinsko polje, Čelo, 450 m, 441478/80834; WL07/4: 29.6.1994, Dolenjska, Novo mesto, Zalog, $178 \mathrm{~m}$, 508548/72226; $163 \mathrm{~m}, 509502 / 72582 ; 164 \mathrm{~m}$, $509644 / 72992$.

(II) - VL99/2: 8.6.2004, Dolenjska, Veliki Gaber, Mišji potok, 330 m, 492867/92686; VL99/3: 11.7.2003, Dolenjska, Polšnik, Golek, 690 m, 497957/100781; WM20/2: 29.4.2010, Štajerska, Razbor (Sevnica), Lisce, 550 m, 521212/102552; XM05/2: 25.5.1995, Štajerska, Dolnja Bistrica, Kolišče Bobri,168 m, 601024/154994.

(III) - WM76/3: 23.8.1995, Štajerska, Mele, 201 m, 578556/169033; WM77/2: 20.4.1995, Štajerska, Lutverci, 212 m, 572088/172307.

(i) - New area of the species distribution: Notranjska region except the Ljubljana basin (Fig. 9). According to the data in the Atlas (Verovnik et al. 2012: pp. 311), the species occurs in Notranjska region only in the northeasternmost part, i.e. in the southwestern part of Ljubljana basin.

(ii) - The presence of species in areas which in the Atlas are designated as "white spots": area around Novo mesto (Fig. 9).

\section{Nymphalis antiopa (Linnaeus, 1758)}

(I) - VM73/2: 29.7.2008, Kamniško-Savinjske Alpe, Veliki Vrh, 1865 m, 475055/134380; WL16/1: 18.5.2004, Dolenjska, Novo mesto, Rajnovšče, 180 m, 513938/69267; WM03/4: 7.4.1987, Štajerska, Velenje, $410 \mathrm{~m}$, 510265/135208; WM12/1: 23.5.1998, Štajerska, Ponikovska planota, Marof, 410 m, 512769/129817; WM42/4: 4.5.1986, Štajerska, Rogaška Slatina, 230 m, 549736/122020.

(II) - VM63/4: 5.5.2003, Kamniško-Savinjske Alpe, Kamniška Bistrica, Dom v Kamniško Bistrici, 587 m, 468679/131432; VM94/2: 18.7.2005, Štajerska, Bele Vode, Senovršnik, 940 m, 495278/ 142158; VM94/4: 18.7.2005, Štajerska, Bele Vode, Srnenski vrh, 952 m, 495894/141672.

(ii) - The presence of species in areas which in the Atlas are designated as "white spots": Kamniško-Savinjske Alps, Velenjsko hills, Ložniško hills, area around Novo mesto.

(iv) - New information on the vertical distribution of species: according to the data in the Atlas (Verovnik et al. 2012: pp. 312-313), the hi- 


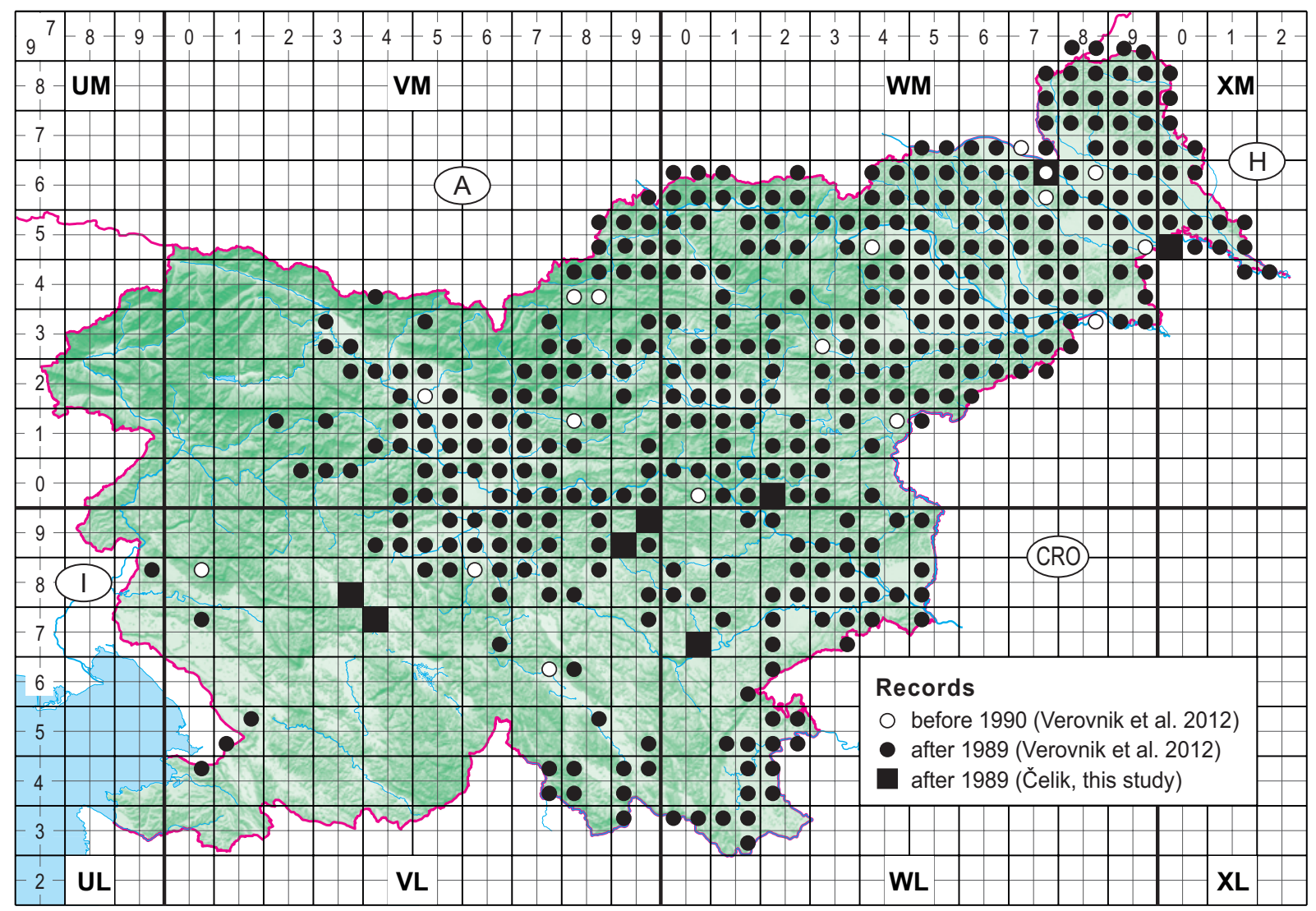

Figure 9: New records on the distribution of Araschnia levana (Linnaeus, 1758) (Čelik, this study) according to the Atlas of butterflies (Lepidoptera: Rhopalocera) of Slovenia (Verovnik et al. 2012: pp. 311).

Slika 9: Novi podatki o razširjenosti vrste Araschnia levana (Linnaeus, 1758) (Čelik, ta študija) glede na podatke v Atlasu dnevnih metuljev (Lepidoptera: Rhopalocera) Slovenije (Verovnik s sod. 2012: str. 311).

ghest confirmed record is from the Vršič pass in the Julian Alps where the species has been observed at an altitude of 1740 meters. My record from Mt. Veliki Vrh in Kamniško-Savinjske Alps (UM83/1) shows that species occurs also at altitudes above 1800 meters.

\section{Nymphalis polychloros (Linnaeus, 1758)}

(I) - VL23/2: 22.4.2006, Primorska, Rakitovec, Podblatice, 509 m, 420443/34840; VL84/3: 6.7.2004, Dolenjska, Kočevska Reka, Mokri potok, 525 m, 487702/47269; VL84/4: 6.7.2004, Dolenjska, Morava, Suhor, 530 m, 490041/43757; WL03/1: 1.6.2004, Dolenjska, Laze pri Predgradu, Kozice, 200 m, 503050/38048.

(II) - UL99/3: 9.6.2006, Primorska, Banjšice, Visoko, 755 m, 399727/98814; 18.5.2006, Primorska, Banjšice, Ježevec, 731 m, 398638/99255; Sleme, 773 m, 399549/100511; VL13/4: 22.4.2006, Primorska, Rakitovec, Podblatice, 494 m, 419993/
34745; VL16/3: 24.6.2005, Primorska, Kras, Griže, 510 m, 418829/67950; VL16/4: 11.6.2003, Primorska, Kras, Brestovica pri Povirju, $405 \mathrm{~m}$, 418675/63611; 400 m, 418317/63885; VL27/4: 21. 6.1998, Notranjska, Nanos, Grmada, 1043 m, 425476/71546; VL44/2: 21.4.2004, Primorska, Ilirska Bistrica, Vrbica, 420 m, 444231/44990; 470 m, 444776/45104; Vrbovo, $470 \mathrm{~m}, 444260 / 45770$; VL44/4: 21.4.2004, Primorska, Ilirska Bistrica, Kuteževo, 460 m, 448520/42930.

(III) - VL16/2: 5.4.1995, Primorska, Sežana, Dol Leskovec, 310 m, 412167/62026; 13.4.1995, Primorska, Sežana, Dol Leskovec, 305 m, 412246/ 62030.

(ii) - The presence of species in areas which in the Atlas are designated as "white spots": Banjška planota, western part of Kočevska region, Kolpa river valley. 


\section{Euphydryas intermedia (Ménétriés, 1859)}

(iii) - New information on locality of the species with very limited distribution in Slovenia: according to the data in the Atlas (Verovnik et al. 2012: pp. 321 ), the species is present only in $0.86 \%$ of all $5 \times 5 \mathrm{~km}$ UTM squares covering the territory of Slovenia, and only in one $5 \times 5 \mathrm{~km}$ UTM square (VM13/3) in the northeastern part of the Julian Alps after 1989. Respecting such limited number of known localities of the species in Slovenia, I add one locality in the northeastern Julian Alps, i.e. VM13/3: 17.7.2009, Julijske Alpe, Pokljuka, Preval, 1690 m, 419158/139033.

\section{Euphydryas maturna (Linnaeus, 1758)}

(I) - VL19/1: 20.6.2007, Primorska, Vojsko, Gačnik, 915 m, 412910/100460.

(ii) - The presence of species in areas which in the Atlas are designated as "white spots": central part of Idrijsko hills. It represents the western limit of the species distribution in Slovenia.

\section{Euphydryas aurinia (Rottemburg, 1775)}

(II) - UM83/1: 18.7.2006, Julijske Alpe, Bovec, Lopa, $2036 \mathrm{~m}$, 384480/137000; UM90/4: 18.5.2006, Primorska, Banjšice, Lohke, $698 \mathrm{~m}$, 399024/102487; Lužarji, 697 m, 399115/103927; VL16/3: 13.5.2005, Primorska, Kras, Griže, 360 m, 418626/68350; VM10/2: 16.6.2006, Primorska, Dolenja Trebuša, Hotenja, 291 m, 412094/104768; 265 m, 411914/105006; WL26/1: 28.6.1994, Dolenjska, Gorjanci, Sv. Miklavž, 941 m, 525182/69956.

(III) - VL15/1: 11.6.2006, Primorska, Lokev, Jirmanec, 658 m, 414621/56055.

(ii) - The presence of species (E. aurinia f. debilis) in areas which in the Atlas are designated as "white spots": westernmost part of the Julian Alps (UM83/1).

\section{Melitaea cinxia (Linnaeus, 1758)}

(II) - UL99/3: 9.6.2006, Primorska, Grgar, Grašišče, 312 m, 397513/96420; 18.6.2002, Primorska, Grgar, Slatna, 360 m, 396483/96604; 9.6.2006, Primorska, Banjšice, Visoko, 739 m, 399860/ 98660; 8.5.2006, Primorska, Banjšice, Visoko, $755 \mathrm{~m}, 399758 / 98732$; 22.6.2006, 9.6.2006, Primorska, Banjšice, Visoko, 755 m, 399727/98814; 22.6.2006, 9.6.2006, Primorska, Banjšice, Tlaka, 705 m, 399466/99091; 702 m, 399593/99162; 18.5.2006, Primorska, Banjšice, Ježevec, $701 \mathrm{~m}$, 398862/99420; 9.6.2006, Primorska, Banjšice,
Kuk, 760 m, 400311/99855; 22.6.2006, Primorska, Banjšice, Sleme, 769 m, 399542/100466; 22.6.2006, 9.6.2006 Primorska, Banjšice, Sleme, 773 m, 399549/100511; VL46/3: 17.6.1994, Notranjska, Cerkniško polje, Dolenjska blata, 548 m, 448706/69904; VL67/4: 15.6.2004, Dolenjska, Mišja dolina, Logarji, 500 m, 468957/75874; VL87/3: 9.6.2004, Dolenjska, Suha krajina, Ravne, 400 m, 485865/77820; VM40/1: 5.7.2004, Gorenjska, Ožbolt nad Zmincem, Sv. Ožbolt, 710 m, 444323/108159.

(III) - VL16/2: 9.6.1995, Primorska, Sežana, Dol Leskovec, 302 m, 412228/62044.

(ii) - The presence of species in areas which in the Atlas are designated as "white spots": Banjška planota.

Melitaea phoebe (Denis \& Schiffermüller, 1775)

(II) - UL99/3: 22.6.2006, Primorska, Banjšice, Kuk, 760 m, 400311/99855; UM91/3: 14.6. 2006, Primorska, Kobarid, Kamno, 184 m, 395768/119558; VL19/1: 17.7.2006, Primorska, Vojsko, Gačnik, 556 m, 412915/100595; VL68/3: 9.6.2009, Dolenjska, Pijava Gorica, Rogovila, 295 m, 466680/88550; VM61/3: 10.6.2004, Gorenjska, Tunjice, Knežji potok, 360 m, 467339/121034.

(III) - VL16/2: 9.6.1995, Primorska, Sežana, Dol Leskovec, 302 m, 412228/62044.

\section{Melitaea trivia (Denis \& Schiffermüller, 1775)}

(I) - VL23/1: 21.6.2002, Primorska, Rakitovec, Kavčič, 600 m, 420415/36188.

(II) - UL99/3: 5.9.2006, Primorska, Banjšice, Visoko, 755 m, 399727/98814; Sleme, 769 m, 399542/100466.

\section{Melitaea didyma (Esper, 1778)}

(I) - VM11/4: 17.7.2006, Primorska, Baška grapa, Zakojca, 639 m, 418318/114023.

(II) - UM91/4: 16.6.2006, Primorska, Doblar, Avško, 655 m, 397757/111482; VL15/1: 25.6.1997, Primorska, Lokev, Mlave, 400 m, 414734/59704; VL16/1: 5.7.2002, Primorska, Kras, Šmarje pri Sežani, 311 m, 412151/66147; VL46/2: 12.6.2003, Notranjska, Trnje (Pivka), Debeli vrh, $640 \mathrm{~m}$, 442256/62620; VL87/4: 9.6.2004, Dolenjska, Suha krajina, Višnje, $400 \mathrm{~m}, 488712 / 73979$; Brezovi Dol, $390 \mathrm{~m}, 489089 / 75096$; Ambrus, $360 \mathrm{~m}$, 486197/75503; WM22/1: 29.6.2005, Štajerska, Frankolovo, Rakova Steza, 330 m, 524113/130770.

(III) - VL16/2: 25.6.1997, Primorska, Sežana, Dol Leskovec, 300 m, 412212/62044; Dane pri Se- 
žani, Koblak, 350 m, 414584/64790; Filipčje Brdo, Prjunščine, 350 m, 414702/65614.

(ii) - The presence of species in areas which in the Atlas are designated as "white spots": Cerkljansko hills.

\section{Melitaea diamina (Lang, 1789)}

(I) - UL99/3: 22.6.2006, Primorska, Banjšice, Visoko, 702 m, 399593/99162; Sleme, 773 m, 399549/100511; UM83/2: 14.6.2006, Primorska, Bovec, Podčela, $343 \mathrm{~m}, 384910 / 131553 ; 341 \mathrm{~m}$, 385026/131913; VM14/4: 10.7.2011, Gorenjska, Krma, Zgornja Radovna, 776 m, 418366/143125.

(II) - VL76/4: 22.6.2004, Dolenjska, Ribnica Prigorica, $480 \mathrm{~m}, 479834 / 63024$; Ugar, $490 \mathrm{~m}$, 478824/64153; Nemška vas, 490 m, 480174/64400; Bukovica, $490 \mathrm{~m}$, 477701/65658; VM40/3: 5.7. 2004, Gorenjska, Škofja Loka, Hrastnica, 430 m, 447100/108928; VM61/3: 10.6.2004, Gorenjska, Tunjice, Tunjščica, 370 m, 468528/120099; VM72/ 3: 21.7.2006, Gorenjska, Krivčevo, Rakov rob, 1133 m, 475975/127144; WL26/1: 28.6.1994, Dolenjska, Gorjanci, Sv. Miklavž, 941 m, 525182/ 69956.

(III) - VL37/4: 12.7.2004, Notranjska, Postojna, Mali Otok, $520 \mathrm{~m}, 436885 / 71151 ; 8.7 .2004$, Notranjska, Postojna, Mali Otok, 521 m, 436151/ 71313.

(ii) - The presence of species in areas which in the Atlas are designated as "white spots": northern part of the Julian Alps, upper part of Soča river valley, Banjška planota, western part of Gorjanci hills. The latter represents the southeastern limit of the species range in Slovenia.

\section{Melitaea aurelia (Nickerl, 1850)}

(I) - UM83/4: 18.7.2006, Julijske Alpe, Bovec, Pl. Goričica, 1368 m, 388055/135430; UM91/3: 14.6.2006, Primorska, Kobarid, Kamno, 170 m, 396080/119044; VL99/2: 8.6.2004, Dolenjska, Veliki Gaber, Mišji potok, 330 m, 493079/92321; WL26/1: 28.6.1994, Dolenjska, Gorjanci, Sv. Miklavž, 941 m, 525182/69956; WM12/1: 25.6.1988, Štajerska, Ponikovska planota, Marof, $410 \mathrm{~m}$, 512776/129825; WM52/4: 22.5.1998, Štajerska, Donačka Gora, 448 m, 556916/124128; XM05/2: 25.5.1995, Štajerska, Dolnja Bistrica, Kolišče Bobri, $168 \mathrm{~m}, 601024 / 154994$.

(II) - UL99/3: 22.6.2006, Primorska, Banjšice, Visoko, $755 \mathrm{~m}, 399727 / 98814$; Sleme, $769 \mathrm{~m}$, 399542/100466; 773 m, 399549/100511; 12.7.2006,
Primorska, Banjšice, Kuk, 760 m, 400311/99855; VL16/4: 11.6.2003, Primorska, Kras, Brestovica pri Povirju, 405 m, 418675/63611; 420 m, 419143/ 63698; VL58/1: 11.5.2001, Notranjska, Goričica pod Krimom, Voščevka, 286 m, 451046/90593; VL84/3: 6.7.2004, Dolenjska, Kočevska Reka, Mokri potok, $525 \mathrm{~m}, 487702 / 47269$; VM10/1: 16.6.2006, Primorska, Dolenja Trebuša, Stopnik, 195 m, 413461/106663; VM94/4: 18.7.2005, Štajerska, Šentvid pri Zavodnju, 934 m, 498634/143192.

(III) - VL16/2: 25.6.1997, Primorska, Sežana, Dol Leskovec, 300 m, 412212/62044.

(ii) - The presence of species in areas which in the Atlas are designated as "white spots": western part of the Julian Alps, eastern part of Dolenjska region, Ložniško hills, area around Donačka gora.

\section{Melitaea britomartis (Assmann, 1847)}

(I) - UL99/3: 22.6.2006, 9.6.2006, Primorska, Banjšice, Visoko, 755 m, 399727/98814; 9.6.2006, Primorska, Banjšice, Visoko, 732 m, 399536/98845; 713 m, 399375/98918; Kuk, 760 m, 400311/99855; 22.6.2006, Primorska, Banjšice, Sleme, $769 \mathrm{~m}$, 399542/100466; 773 m, 399549/100511; VL03/1: 13.6.1987, Primorska, Koprska Brda, Koštabona, 75 m, 402544/37214; VM10/2: 16.6.2006, Primorska, Dolenja Trebuša, Hotenja, 291 m, 412094/ 104768; 265 m, 411914/105006.

(II) - VL16/4: 11.6.2003, Primorska, Kras, Brestovica pri Povirju, 395 m, 418432/63811.

(ii) - The presence of species in areas which in the Atlas are designated as "white spots": Banjška planota, Koprska Brda region.

\section{Melitaea athalia (Rottemburg, 1775)}

(II) - UM90/4: 30.6.2006, Primorska, Banjšice, Breščaki, 632 m, 398146/101314; VL16/1: 11. 6.2003, Primorska, Kras, Tomaj, 340 m, 411103/ 68920; VL46/2: 12.6.2003, Notranjska, Trnje (Pivka), Debeli vrh, $630 \mathrm{~m}, 442230 / 62502 ; 620 \mathrm{~m}$, 442315/62512; VL83/1: 6.7.2004, Dolenjska, Srobotnik ob Kolpi, Rački potok, 250 m, 485058/ 38990; VM33/2: 17.7.2005, Julijske Alpe, Jelovica, Pl. Talež, 711 m, 432175/132158; VM73/4: 21.7. 2009, Kamniško-Savinjske Alpe, Dleskovška planota, Pl. Ravne, 1520 m, 477346/134194; WL26/1: 28.6.1994, Dolenjska, Gorjanci, Sv. Miklavž, $941 \mathrm{~m}, 525182 / 69956$.

(III) - VL83/4: 6.7.2004, Dolenjska, Petrina, Grivački jarek, 310 m, 488080/35827; 
XM15/2: 22.8.1995, Prekmurje, Petišovci, 160 m, $611632 / 153802$.

\section{Limenitis populi (Linnaeus, 1758)}

(I) - VL67/2: 19.7.2009, Notranjska, Bloška planota, Bloški hrib, $772 \mathrm{~m}, 460827 / 71587$; VL77/1: 22.6.2004, Dolenjska, Velike Lašče, Grivka, 560 m, 471440/76687; VM94/4: 18.7.2005, Štajerska, Zavodnje, Vovke, 590 m, 499460/141500.

(II) - UM91/4: 16.6.2006, Primorska, Doblar, Lepenka, 344 m, 399024/111763; VL68/3: 21.6.2001, Dolenjska, Pijava Gorica, Podblato, $305 \mathrm{~m}, 469040 / 89807$.

(III) - WL26/1: 28.6.1994, Dolenjska, Gorjanci, Sv. Miklavž, 941 m, 525182/69956.

(ii) - The presence of species in areas which in the Atlas are designated as "white spots": extensive area between the eastern part of Notranjska region and Suha krajina region.

(iv) - New information on species occurrence in the Ljubljana basin: according to the data in the Atlas (Verovnik et al. 2012: pp. 343), the species has not been observed in this region since 1990. My record from Pijava Gorica (VL68/3) shows that in the surroundings of Ljubljana the species was also found in the last decade.

\section{Limenitis camilla (Linnaeus, 1764)}

(I) - UM90/4: 30.6.2006, Primorska, Banjšice, Bate, 597 m, 397290/101950; Kanalski Vrh, 350 m, 395765/104840; VL06/1: 11.6.2003, Primorska, Kras, Pliskovica, 220 m, 404645/70288; VM11/4: 17.7.2006, Primorska, Baška grapa, Zakojca, $612 \mathrm{~m}, 417675 / 114396$.

(II) - UL99/3: 30.6.2006, Primorska, Grgarske Ravne, Zabrdo, 460 m, 398089/98030; VL67/ 2: 19.7.2009, Notranjska, Bloška planota, Bloški hrib, $772 \mathrm{~m}, 460827 / 71587 ; 772 \mathrm{~m}, 460790 /$ 71745; 758 m, 460790/71935; VL84/1: 6.7.2004, Dolenjska, Kočevska Reka, Mokri potok, 540 m, 485181/46829; VM40/3: 5.7.2004, Gorenjska, Škofja Loka, Hrastnica, 535 m, 446363/107359; VM82/2: 21.7.2006, Gorenjska, Gornji Grad, Šmiklavž, 945 m, 481490/123970.

(ii) - The presence of species in areas which in the Atlas are designated as "white spots": Kras, Cerkljansko hills.

\section{Limenitis reducta (Staudinger, 1901)}

(I) - VL37/3: 1.7.1993, Notranjska, Planinsko polje, Sv. Jedert, 445 m 440293/80204; WM12/1:
20.6.1998, Štajerska, Ponikovska planota, Marof, 394 m, 512755/129727; 21.7.2002, Śtajerska, Ponikovska planota, Marof, 410 m, 512776/129825.

(II) - VL06/1: 29.5.2002, Primorska, Kras, Pliskovica, $230 \mathrm{~m}, 404633 / 70318$; VL16/1: 29. 5.2002, Primorska, Kras, Tomaj, 380 m, 410968/ 68755; VL38/4: 8.6.1993, Notranjska, Planinsko polje, Grčarevec, 445 m, 439539/81106; VL46/2: 12.6.2003, Notranjska, Trnje (Pivka), Debeli vrh, 620 m, 442315/62512; VL69/4: 5.6.2001, Dolenjska, Šmarje-Sap, Huda polica, $318 \mathrm{~m}, 469470$ / 91360.

(iv) - New information on species occurrence in the foothills of the Eastern Karavanke Mountains (i.e. Velenjsko and Konjiško hills, Ložniško and Hudinjsko hills): the species distribution map in the Atlas (Verovnik et al. 2012: pp. 347) indicates that in the foothills of the Eastern Karavanke Mountains the species was observed at only one locality, and only before 1989. The species is still present in this region (Čelik, pers. observ.) although my record from Ponikovska planota (WM12/1) is ten years old.

\section{Neptis sappho (Pallas, 1771)}

(II) - VL99/4: 26.5.2004, Dolenjska, Moravška gora, Červatec, 590 m, 499290/95993.

\section{Neptis rivularis (Scopoli, 1763)}

(I) - VL19/1: 20.6.2007, Primorska, Vojsko, Gačnik, 919 m, 413545/100100; 17.7.2006, Primorska, Gorenja Trebuša, Makčeva grapa, $556 \mathrm{~m}$, 410707/100760; VL67/3: 22.6.2004, Dolenjska, Rašica, Dolščaki, 500 m, 467402/78160; VL94/2: 6.7.2004, Dolenjska, Podstene pri Kostelu, Potok, 300 m, 491880/41854; 335 m, 490680/42174; WM03/1: 18.7.2005, Štajerska, Šoštanj, Kurja vas, $408 \mathrm{~m}, 500692 / 138400$.

(II) - VL83/4: 6.7.2004, Dolenjska, Petrina, Grivački jarek, 310 m, 488080/35827; VM10/2: 16.6.2006, Primorska, Dolenja Trebuša, Hotenja, $291 \mathrm{~m}, 412094 / 104768 ; 265 \mathrm{~m}, 411914 / 105006$; Log, 211 m, 413968/105963; VM94/4: 18.7.2005, Štajerska, Zavodnje, Mazetov graben, $555 \mathrm{~m}$, 500250/141326; Vovke, 590 m, 499460/141500.

(III) - VM41/4: 5.7.2004, Gorenjska, Škofja Loka, Hrastnica, 380 m, 447303/111248.

(ii) - The presence of species in areas which in the Atlas are designated as "white spots": western part of Dolenjska region, Kolpa river valley. 
Apatura ilia (Denis \& Schiffermüller, 1775)

(II) - VL84/4: 6.7.2004, Dolenjska, Morava, Suhor, $530 \mathrm{~m}, 490041 / 43757$; VM90/4: 23.6.2011, Dolenjska, Renke, Kleviška špica, $340 \mathrm{~m}, \quad 496808 / 104803$; WM23/2: 29.6.2005, Štajerska, Frankolovo, Straža pri Dolu, $341 \mathrm{~m}$, 524180/132467; WM67/2: 12.6.1996, Štajerska, Zgornje Konjišče, 225 m, 563384/175172.

\section{Apatura iris (Linnaeus, 1758)}

(I) - UL97/3: 1.8.2005, Primorska, Kras, Novelo, 360 m, 395980/79664; UM90/4: 30.6.2006, Primorska, Kanal, Kanalski Vrh, 350 m, 395765/ 104840.

(II) - VL45/4: 9.7.1994, Notranjska, Koritnice, Suha reber, $671 \mathrm{~m}, 445539 / 51767$; VM11/4: 17.7.2006, Primorska, Baška grapa, Hudajužna, 400 m, 416475/115118; WM86/4: 4.5.1995, Štajerska, Vučja vas, $185 \mathrm{~m}, 585857 / 162473$.

(III) - VM40/3: 5.7.2004, Gorenjska, Sora, Ločnica, 425 m, 450291/107776.

(ii) - The presence of species in areas which in the Atlas are designated as "white spots": Banjška planota, Kras.

\section{Pararge aegeria (Linnaeus, 1758)}

(I) - VM11/4: 17.7.2006, Primorska, Baška grapa, Zakojca, 612 m, 417675/114396; Obloke, $464 \mathrm{~m}, 416304 / 115252$.

(II) - UM90/1: 30.6.2006, Primorska, Kanalski Kolovrat, Čolnica, 489 m, 393760/106520; UM91/4: 16.6.2006, Primorska, Doblar, Lepenka, $344 \mathrm{~m}$, 399024/111763; Osredek, $495 \mathrm{~m}$, 398690/112268; VL13/4: 22.4.2006, Primorska, Rakitovec, 481 m, 419757/34598; VL15/1: 11.6. 2006, Primorska, Lokev, Jirmanec, 632 m, 414011/ 56337; $611 \mathrm{~m}, 414279 / 56420$; $551 \mathrm{~m}, 413866 / 56478$; VL15/4: 8.5.2009, Primorska, Kozina, Krvavi potok, 496 m, 415930/53117; VL16/3: 26.9.2005, Primorska, Kras, Griže, 479 m, 418704/67946; $422 \mathrm{~m}, \quad 418441 / 68492 ; 14.7 .2005$, Primorska, Kras, Griže, 467 m, 418630/68030; 13.5.2005, Primorska, Kras, Griže, 428 m, 418536/68491; VL19/1: 20.6.2007, Primorska, Vojsko, Gačnik, 919 m, 413545/100100; VL48/2: 18.5.1993, Notranjska, Planinsko polje, Pod Stenami, $474 \mathrm{~m}$, 441352/81210; VL67/3: 15.6.2004, Dolenjska, Rašica, Robarica, 530 m, 466402/77630; VL67/4: 22.6.2004, Dolenjska, Podstrmec, Kozmanjka, 610 m, 466521/72097; 600 m, 466423/72370; Podleščica, 560 m, 467439/72781; VL84/4: 19.5.2011, Dolenjska, Gornja Briga, Prežulje, $620 \mathrm{~m}$,
485837/43203; VM94/4: 18.7.2005, Štajerska, Zavodnje, Mazetov graben, 555 m, 500250/141326.

(III) - VL16/2: 8.4.1995, Primorska, Sežana, Dol Leskovec, 310 m, 412167/62026; 13.4.1995, Primorska, Sežana, Dol Leskovec, 305 m, 412246/ 62030; VM71/2: 22.8.2003, Gorenjska, Radomlje, Rova, 339 m, 471277/115029.

\section{Lasiommata megera (Linnaeus, 1767)}

(II) - VL13/4: 22.4.2006, Primorska, Maršiči, 125 m, 415367/34988; VL15/1: 11.5.2008, Primorska, Sežana, Lipica, 378 m, 412860/58600; 374 m, 412965/59090; $380 \mathrm{~m}, 412895 / 60012$; WL17/3: 11.5.2004, Dolenjska, Novo mesto, Srednje Grčevje, $480 \mathrm{~m}$, 515938/79264; WM86/4: 4.5.1995, Štajerska, Vučja vas, 185 m, 585857/162473.

\section{Lasiommata petropolitana (Fabricius, 1787)}

(I) - UM72/3: 4.6.1998, Primorska, Breginj, Prekopa, 876 m, 378245/126830; VM53/2: 21.7. 2010, Kamniško-Savinjske Alpe, Storžič, 1625 m, 453907/133783.

(II) - VM63/2: 16.7.2005, Kamniško-Savinjske Alpe, Grintovec, Spodnji Dolci, $1850 \mathrm{~m}$, 464340/134200.

(ii) - The presence of species in areas which in the Atlas are designated as "white spots": western part of Kamniško-Savinjske Alps.

\section{Lasiommata maera (Linnaeus, 1758)}

(I) - VL87/4: 9.6.2004, Dolenjska, Suha krajina, Ambrus, 360 m, 486197/75503; VM10/2: 16.6.2006, Primorska, Dolenja Trebuša, Hotenja, $291 \mathrm{~m}, 412094 / 104768 ; 265$ m, 411914/105006; WM12/1: 30.8.1987, Štajerska, Ponikovska planota, Marof, 410 m, 512776/129825.

(II) - UM90/4: 30.6.2006, Primorska, Banjšice, Breščaki, $632 \mathrm{~m}, 398146 / 101314 ;$ VL25/2: 21.5.2009, Notranjska, Brkini, Sušica, $411 \mathrm{~m}$, 422729/55848; 18.5.2009, Notranjska, Brkini, Sušica, 409 m, 423006/55898; VL46/2: 12.6.2003, Notranjska, Trnje (Pivka), Sv. Trojica, $941 \mathrm{~m}$, 443244/64550; VL99/2: 8.6.2004, Dolenjska, Veliki Gaber, Mišji potok, 330 m, 493079/92321; VL99/4: 17.5.2011, Dolenjska, Moravška Gora, Červatec, 593 m, 499041/96028; VM32/4: 17.7.2005, Gorenjska, Jelovica, Dražgoše, 1020 m, 438000/123300; VM40/2: 23.6.2004, Gorenjska, Polhograjsko hribovje, M. Božna, 480 m, 445066/105937.

(ii) - The presence of species in areas which in the Atlas are designated as "white spots": Banj- 
ška planota, Suha Krajina region, Ložniško and Hudinjsko hills.

\section{Lopinga achine (Scopoli, 1763)}

(I) - VM11/4: 17.7.2006, Primorska, Baška grapa, Zakojca, 639 m, 418318/114023; WM04/1: 1.7.2011, Vzhodne Karavanke, Kozji hrbet, 578 m, 502926/149634.

(II) - UL99/3: 18.6.2002, Primorska, Grgar, Slatna, 360 m, 396483/96604; 22.6.2006, Primorska, Banjšice, Visoko, 724 m, 399990/98688; UM83/2: 14.6.2006, Primorska, Bovec, Podčela, $341 \mathrm{~m}, 385026 / 131913$; VL46/2: 12.6.2003, Notranjska, Trnje (Pivka), Debeli vrh, $620 \mathrm{~m}$, 442315/62512; VL84/3: 6.7.2004, Dolenjska, Kočevska Reka, Mokri potok, 525 m, 487702/47269; VL94/2: 6.7.2004, Dolenjska, Podstene pri Kostelu, Gorenji Potok, 335 m, 490680/42174; VM10/2: 16.6.2006, Primorska, Dolenja Trebuša, Hotenja, 291 m, 412094/104768; 265 m, 411914/105006.

(ii) - The presence of species in areas which in the Atlas are designated as "white spots": Cerkljansko hills.

(iv) - New information on the vertical distribution of species: according to the data in the Atlas (Verovnik et al. 2012: pp. 364), the species occurs at heights up to $1400 \mathrm{~m}$ a. s. l. only in the Julian Alps. I found the species within the upper limit of montane zone also in Kamniško-Savinjske Alps, i.e. VM53/2: 21.7.2010, Kamniško-Savinjske Alpe, Storžič, Pl. Mala Poljana, 1400 m, 452455/134035.

\section{Coenonympha tullia (Müller, 1764)}

As in the period from 2002 to 2011 the species in Slovenia was not observed, it was classified as presumed extinct (Čelik et al. 2005). It has been rediscovered in June 2011 (Čelik 2011) at the locality where it had been occurred until the end of the 80th-s of the last century (Čelik \& Rebeušek 1996), i.e. in the surroundings of Kranjska Gora (Čelik 2012).

(iii) and (iv) - Update of the records in the UTM square VM04/1 on the distribution map of species in the Atlas (Verovnik et al. 2012: pp. 367): based on the observations of adults at the locality in the surroundings of Kranjska Gora in 2011 and 2012 (Čelik, unpublished), the minimum abundance class (i.e. the maximum number of individuals observed on a single occasion at any location within UTM square after 1989, cf.
Verovnik et al. 2012: pp. 76) should be replaced with a maximum class, i.e. more than 20 specimens/1 occasion observed after 1989.

\section{Coenonympha oedippus (Fabricius, 1787)}

(II) - UL99/3: 30.6.2006, 22.6.2006, Primorska, Grgarske Ravne, Zabrdo, 466 m, 398089/ 98030 .

(ii) - The presence of species in areas which in the Atlas are designated as "white spots": Banjška planota. It represents the northwestern limit of the species range in Slovenia.

\section{Coenonympha arcania (Linnaeus, 1761)}

(I) - VM11/4: 17.7.2006, Primorska, Baška grapa, Zakojca, 639 m, 418318/114023; Hudajužna, 424 m, 416263/114968; Obloke, 455 m, 416278/115122.

(II) - UM90/4: 30.6.2006, Primorska, Banjšice, Breščaki, $632 \mathrm{~m}$, 398146/101314; 30.6.2006, Primorska, Kanal, Kanalski Vrh, 350 m, 395765/ 104840; VL16/1: 29.5.2002, Primorska, Kras, Tomaj, 380 m, 410968/68755; 335 m, 411141/68960; 11.6.2003, Primorska, Kras, Tomaj, 340 m, 411103/ 68920; 340 m, 411137/68931; VL16/4: 11.6.2003, Primorska, Kras, Brestovica pri Povirju, 395 m, 418432/63811; VL69/4: 5.6.2001, Dolenjska, Šmarje-Sap, Huda polica, 306 m, 469382/91430; VL76/4: 22.6.2004, Dolenjska, Ribnica, Prigorica, 480 m, 479834/63024; Ugar, 490 m, 478824/64153; VL87/3: 9.6.2004, Dolenjska, Fužina, Grintovec, 310 m, 487004/78823; VM82/4: 21.7.2006, Kamniško-Savinjske Alpe, Menina planina, Šavnica, 1422 m, 489550/122680; 1415 m, 489305/122870.

(III) - VL16/2: 25.6.1997, Primorska, Sežana, Dol Leskovec, 300 m, 412212/62044; Dane pri Sežani, Koblak, 350 m, 414584/64790; 9.6.1995, Primorska, Sežana, Dol Leskovec, 302 m, 412228/ 62044.

(ii) - The presence of species in areas which in the Atlas are designated as "white spots": Cerkljansko hills.

(iv) - New information on the vertical distribution of species: according to the data in the Atlas (Verovnik et al. 2012: pp. 370), the species occurs at heights up to $1700 \mathrm{~m}$ a. s. l. only in the Eastern Karavanke Mountains. I found the species at altitudes between 1600 and 1700 meters also in the Kamniško-Savinjske Alps, i.e. VM53/2: 21.7.2010, Kamniško-Savinjske Alpe, Storžič, 1625 m, 453907/133783; 1612 m, 453730/133838. 


\section{Coenonympha glycerion (Borkhausen, 1788)}

(I) - VL16/2: 25.6.1997, Primorska, Kras, Filipčje Brdo, $350 \mathrm{~m}, 414702 / 65614$; WL26/1: 28.6.1994, Dolenjska, Gorjanci, Sv. Miklavž, $941 \mathrm{~m}, 525182 / 69956$.

(II) - VL47/1: 27.5.2010, Notranjska, Planinsko polje, Liplje, 447 m, 442615/78360; VL67/3: 22.6.2004, Dolenjska, Rašica, Podlog, $480 \mathrm{~m}$, 469946/78258; 15.6.2004, Dolenjska, Rašica, Podlog, 480 m, 469860/78278; VL76/4: 22.6.2004, Dolenjska, Ribnica, Prigorica, 480 m, 479834/63024; Ugar, 490 m, 478824/64153; Nemška vas, 490 m, 480174/64400; Bukovica, 490 m, 477701/65658; VL84/3: 6.7.2004, Dolenjska, Kočevska Reka, Mokri potok, 525 m, 487702/47269; VL87/4: 9.6. 2004, Dolenjska, Suha krajina, Brezovi Dol, 390 m, 489089/75096; VL97/2: 9.6.2004, Dolenjska, Suha krajina, Veliko Lipje, 310 m, 493787/73768.

(ii) - The presence of species in areas which in the Atlas are designated as "white spots": Kras, Gorjanci hills. According to the comments in the Atlas (cf. Verovnik et al. 2012: pp. 372), the species is absent from the lowland areas in the Primorska region. My record from Kras (VL16/2) shows that species occurs in Primorska region also in the lowlands.

\section{Coenonympha gardetta (De Prunner, 1798)}

(II) - UM83/1: 18.7.2006, Julijske Alpe, Bovec, Lopa, 2036 m, 384480/137000; UM92/1: 18.7.2003, Julijske Alpe, Drežniške Ravne, Krasji vrh, 1620 m, 391160/128425; 1665 m, 391240/ 128464.

(ii) - The presence of species in areas which in the Atlas are designated as "white spots": the westernmost part of the Julian Alps (UM83/1). It represents the western limit of the species range in Slovenia.

\section{Coenonympha pamphilus (Linnaeus, 1758)}

(I) - VM54/4: 20.7.2006, Zahoden Karavanke, Košuta, Pl. Spodnje Dolge njive, 1536 m, $456080 / 144040$.

(II) - UM90/4: 30.6.2006, Primorska, Banjšice, Breščaki, 632 m, 398146/101314; Kanalski Vrh, 571 m, 397110/104200; 18.5.2006, Primorska, Banjšice, Lohke, 698 m, 399024/102487; VM10/2: 16.6.2006, Primorska, Dolenja Trebuša, Hotenja, $291 \mathrm{~m}, 412094 / 104768 ; 265 \mathrm{~m}, 411914 / 105006$; WL25/1: 19.5.2004, Dolenjska, Bela Krajina, Metlika, 190 m, 522071/56666.

\section{Aphantopus hyperantus (Linnaeus, 1758)}

(I) - VL06/1: 5.7.2002, Primorska, Kras, Podbreg, 220 m, 404742/70251; VL19/1: 20.6.2007, Primorska, Vojsko, Gačnik, 911 m, 412900/100630; VL26/3: 25.6.1997, Primorska, Senožeče, Laže, 698 m, 428111/66702; VL99/3: 11.7.2003, Dolenjska, Sopota, Golek, 690 m, 497957/100781; 680 m, 498250/101025; WM03/1: 18.7.2005, Štajerska, Šoštanj, Kurja vas, 408 m, 500692/138400; WM04/1: 1.7.2011, Vzhodne Karavanke, Vrhe, Kozji hrbet, 578 m, 502926/149634.

(II) - UL99/3: 18.6.2002, Primorska, Grgar, Slatna, 360 m, 396483/96604; UM90/1: 30.6.2006, Primorska, Kanalski Kolovrat, Čolnica, 489 m, 393760/106520; Lig, 607 m, 392810/107048; UM 90/4: 30.6 .2006 , Primorska, Kanal, Kanalski Vrh, 350 m, 395765/104840; VL68/3: 2.7.2009, Dolenjska, Pijava Gorica, Gorenje Blato, 306 m, 468713/90410; 16.7.2008, Dolenjska, Pijava Gorica, Gorenje Blato, 310 m, 468745/90390; 6.7.2008, Dolenjska, Pijava Gorica, Rogovila, $295 \mathrm{~m}$, 466680/88550; Podblato, 305 m, 469020/89750; Gorenje Blato, $310 \mathrm{~m}, 468745 / 90390 ; 7.7 .2001$, Dolenjska, Pijava Gorica, Gorenje Blato, 306 m, 468707/90454; 4.7.2001, Dolenjska, Pijava Gorica, Podblato, 305 m, 469030/89802; 30.6.2001, Dolenjska, Pijava gorica, Gorenje Blato, $306 \mathrm{~m}$, 468684/90460; VL79/2: 12.7.2003, Dolenjska, Dobje, Črna dolina, 350 m, 473810/92850.

(III) - VM94/4: 18.7.2005, Štajerska, Zavodnje, Kot, 713 m, 498950/141288; Mazetov graben, 555 m, 500250/141326; Bele Vode, Srnenski vrh, $952 \mathrm{~m}, 495894 / 141672$.

(i) - New area of the species distribution: Idrijsko hills. Note: according to the authors' comments in the Atlas (cf. Verovnik et al. 2012: pp. 380), the species is absent from Idrijsko region.

(ii) - The presence of species in areas which in the Atlas are designated as "white spots": western part of Kras, Posavsko hills, Velenjsko hills, foothills of the Eastern Karavanke Mountains.

\section{Maniola jurtina (Linnaeus, 1758)}

(II) - UM90/1: 30.6.2006, Primorska, Kanalski Kolovrat, Lig, 607 m, 392810/107048; Britof , 264 m, 391104/107092; VL28/4: 26.6.2002, Primorska, Podkraj (Ajdovščina), Križna gora, 779 m, 425875/81106; VL76/4: 22.6.2004, Dolenjska, Ribnica, Prigorica, 480 m, 479834/63024; Nemška vas, 490 m, 480174/64400; Bukovica, 490 m, 477701/65658; VM10/2: 16.6.2006, Primorska, Dolenja Trebuša, Hotenja, 291 m, 412094/104768; 
265 m, 411914/105006; Stopnik, 211 m, 413968/ 105963; VM32/1: 17.7.2005, Julijske Alpe, Jelovica, Goška ravan, 1001 m, 433570/130640; VM 41/4: 5.7.2004, Gorenjska, Škofja Loka, Hrastnica, 380 m, 447303/111248;VM90/1: 11.7.2003, Štajerska, Zagorje ob Savi, Mošenik, $290 \mathrm{~m}$, 494192/106080; WL07/4: 29.6.1994, Dolenjska, Novo mesto, Zalog, 163 m, 509502/72582; 28.6. 1994, Dolenjska, Novo mesto, Prečna, 164 m, 509644/72992.

(III) - VL16/2: 9.6.1995, Primorska, Sežana, Dol Leskovec, 302 m, 412228/62044; VL83/4: 6.7.2004, Dolenjska, Petrina, Grivački jarek, 310 m, 488080/35827; WM57/4: 12.6.1996, Stajerska, Sladki Vrh, 232 m, 558564/172964.

(ii) - The presence of species in areas which in the Atlas are designated as "white spots": central part of Kočevsko region, area around Novo Mesto.

\section{Erebia ligea (Linnaeus, 1758)}

(I) - VL84/3: 6.7.2004, Dolenjska, Kočevska Reka, Mokri potok, 525 m, 487702/47269; WM 04/1: 1.7.2011, Vzhodne Karavanke, Vrhe, Kozji hrbet, $578 \mathrm{~m}, 502926 / 149634$.

(II) - VL85/4: 25.6.1994, Dolenjska, Kočevje, Stojna, 929 m, 485878/53646; VM13/3: 17.7.2009, Julijske Alpe, Pokljuka, Debela peč, 1690 m, 419158/139033; VM32/4: 17.7.2005, Julijske Alpe, Jelovica, Dražgoše, 1020 m, 438000/123300; VM94/4: 18.7.2005, Štajerska, Bele Vode, Srnenski vrh, 952 m, 495894/141672; Andrejev dom na Slemenu, 1087 m, 496380/143168; Šentvid pri Zavodnju, 934 m, 498634/143192; 990 m, 498396/ 143380.

(III) - VM94/2: 18.7.2005, Štajerska, Bele Vode, Senovršnik, 940 m, 495278/142158.

(ii) - The presence of species in areas which in the Atlas are designated as "white spots": Velenjsko hills, foothills of the Eastern Karavanke Mountains, southern part of Dolenjska region. The latter represents the species' southeastern distribution limit in Slovenia.

\section{Erebia euryale (Esper, 1805)}

(II) - VM13/3: 17.7.2009, Julijske Alpe, Pokljuka, Pl. Klek, 1570 m, 420280/139515.

(III) - UM92/1: 18.7.2003, Julijske Alpe, Drežniške Ravne, Pl. Zaprikraj, 1480 m, 392890/ 127700; 1450 m,393070/127950; Krasjivrh, 1620 m, 391160/128425; VM12/2: 25.7.2005, Julijske Alpe,
Rodica, Zadnja Suha, 1573 m, 411954/121980; Pl. Suha, 1388 m, 412041/122509; Javorje, 1115 m, 412850/123250; $1050 \mathrm{~m}, 413041 / 123508$.

(iv) - New information on species occurrence in the southwestern Julian Alps: according to the distribution map in the Atlas (Verovnik et al. 2012: pp. 389), the species was observed in this region only at one locality, and has not been found there since 1990. My records from Polovnik Mountains (UM92/1) show that species was present in this part of the Julian Alps as late as 2003.

\section{Erebia manto (Denis \& Schiffermüller, 1775)}

(II) - UM92/1: 18.7.2003, Julijske Alpe, Drežniške Ravne, Planina Zaprikraj, 1550 m, 392387/ 127935; Krasji vrh, 1685 m, 391870/128236; Veliki vrh, $1620 \mathrm{~m}, 391160 / 128425$.

(III) - VM12/2: 25.7.2005, Julijske Alpe, Rodica, Zadnja Suha, 1573 m, 411954/121980; Pl. Suha, $1388 \mathrm{~m}, 412041 / 122509$.

(ii) - The presence of species in areas which in the Atlas are designated as "white spots": southwestern part of the Julian Alps.

\section{Erebia epiphron (Knoch, 1783)}

(II) - UM83/1: 18.7.2006, Julijske Alpe, Bovec, Lopa, 2036 m, 384480/137000; 2146 m, $383667 / 137165$.

(III) - UM83/3: 18.7.2006, Julijske Alpe, Bovec, Črnelski Vršiči, 2157 m, 386303/137855; $2115 \mathrm{~m}, 386560 / 137970$.

\section{Erebia aethiops (Esper, 1777)}

(I) - UM90/4: 30.6.2006, Primorska, Banjšice, Bate, 597 m, 397290/101950; WM12/1: 30.8.1987, Štajerska, Ponikovska planota, Marof, $410 \mathrm{~m}$, 512776/129825.

(II) - UL99/3: 12.7.2006, Primorska, Banjšice, Visoko, 734 m, 399943/98658; 755 m, 399727/ 98814; $713 \mathrm{~m}, \quad 399375 / 98918 ;$ Tlaka, $705 \mathrm{~m}$, 399466/99091; Kuk, 760 m, 400311/99855; Sleme, $769 \mathrm{~m}, 399542 / 100466$; 25.7.2005, Primorska, Banjšice, Pičulini, 717 m, 399978/98784; 23.8.2006, Primorska, Banjšice, Visoko, $713 \mathrm{~m}$, 399375/98918; VM11/4: 17.7.2006, Primorska, Baška grapa, Zakojca, 639 m, 418318/114023; Obloke, $464 \mathrm{~m}, 416304 / 115252$.

(ii) - The presence of species in areas which in the Atlas are designated as "white spots": Banjška planota. 
Erebia medusa (Denis \& Schiffermüller, 1775)

(I) - UM72/3: 4.6.1998, Primorska, Breginj, Prekopa, 876 m, 378245/126830; VM11/4: 17.7.2006, Primorska, Baška grapa, Hudajužna, $424 \mathrm{~m}, 416263 / 114968$.

(II) - VL15/1: 11.6.2006, Primorska, Lokev, Jirmanec, $658 \mathrm{~m}, 414621 / 56055 ; 611 \mathrm{~m}, 414279 /$ 56420; 421 m, 413383/56889; VL16/3: 10.6.2005, Primorska, Kras, Griže, $468 \mathrm{~m}, 418613 / 68056$; 10.6.2005, 13.5.2005, Primorska, Kras, Griže, 360 m, 418626/68350; VL27/4: 21.6.1998, Notranjska, Nanos, Grmada, 1043 m, 425476/71546; VL66/2: 24.5.1994, Notranjska, Loška dolina, Vrhnika pri Ložu, 582 m, 462187/61865; VL74/3: 19.5.2011, Dolenjska, Bezgarji, 400 m, 477267/ 46097; VM13/3: 17.7.2009, Julijske Alpe, Pokljuka, Debela peč, 1690 m, 419158/139033; Klečica, 1862 m, 419150/139696; VM23/1: 17.7.2009, Julijske Alpe, Pokljuka, Pl. Klek, 1510 m, 420410/ 139143.

(ii) - The presence of species in areas which in the Atlas are designated as "white spots": Cerkljansko hills.

\section{Erebia gorge (Hübner, 1804)}

(II) - VM13/3: 17.7.2009, Julijske Alpe, Pokljuka, Klečica, 1880 m, 418892/139545; VM53/2: 21.7.2010, Kamniško-Savinjske Alpe, Storžič, 1895 m, 454302/133963; 2050 m, 454491/134010; 2118 m, 454637/134275; VM63/2: 16.7.2005, Kamniško-Savinjske Alpe, Grintovec, Spodnji Dolci, 1850 m, 464340/134200; VM73/4: 21.7.2009, Kamniško-Savinjske Alpe, Dleskovška planota, Dleskovec, 1795 m, 476057/134883.

(III) - UM83/3: 18.7.2006, Julijske Alpe, Bovec, Črnelski Vršiči, 1740 m, 387130/136950; 2008 m, 385600/137140; 1780 m, 387060/137280; $2028 \mathrm{~m}, 386050 / 137430 ; 2017 \mathrm{~m}, 386750 / 137840$, 2157 m, 386303/137855; VM62/1: 15.7.2005, Kamniško-Savinjske Alpe, Ambrož pod Krvavcem, Veliki Zvoh, 1910 m, 464874/129340.

(ii) - The presence of species in areas which in the Atlas are designated as "white spots": eastern part of the Julian Alps.

(iv) - New information on species occurrence in western and central part of the Kamniško-Savinjske Alps: the species distribution map in the Atlas (Verovnik et al. 2012: pp. 403) indicates that species has not been observed in those regions since 1990. My records from Mt. Storžič (VM53/2), Mt. Grintovec (VM63/2) and Mt. Kr- vavec (VM62/1) show that the species is still present in the western and central part of Kamniško-Savinjske Alps.

\section{Erebia pronoe (Esper, 1780)}

(II) - UM82/1: 6.8.2004, Julijske Alpe, Kobariški Stol, Na Verilih, 1550 m, 382017/126977.

(III) - VM12/2: 25.7.2005, Julijske Alpe, Rodica, Pl. Suha, 1388 m, 4120417122509; VM53/2: 21.7.2010, Kamniško-Savinjske Alpe, Storžič, Pl. Javornik, 1574 m, 453255/133837; Psica, 1570 m, 453448/133846.

(ii) - The presence of species in areas which in the Atlas are designated as "white spots": southwestern edge of the Julian Alps.

(iv) - New information on species occurrence in western part of the Kamniško-Savinjske Alps: according to the distribution map in the Atlas (Verovnik et al. 2012: pp. 407), the species has not been observed in that region since 1990. My records from Mt. Storžič (VM53/2) show that species is still present in the western part of the Kamniško-Savinjske Alps.

\section{Erebia stiria (Godart, 1824)}

(I) - VM32/4: 17.7.2005, Julijske Alpe, Jelovica, Dražgoše, $1020 \mathrm{~m}, 438000 / 123300$.

(II) - UL99/3: 30.6.2006, Primorska, Grgarske Ravne, Zabrdo, 460 m, 398089/98030; VL19/1: 17.7.2006, Primorska, Vojsko, Gačnik, $556 \mathrm{~m}, 412915 / 100595$.

(ii) - The presence of species in areas which in the Atlas are designated as "white spots": eastern part of the Julian Alps, Banjška planota, Idrijsko hills.

\section{Erebia oeme (Hübner, 1804)}

(I) - VM73/4: 21.7.2009, Kamniško-Savinjske Alpe, Dleskovška planota, Dleskovec, $1830 \mathrm{~m}$, 476423/134110.

(i) - New area of the species distribution: Kamniško-Savinjske Alps (Fig. 10). Note: On the species distribution map in the Atlas (Verovnik et al. 2012: pp. 415), there is only one record in the Kamniško-Savinjske Alps, and it is classified as a wrong/questionable record (gray circle, cf. Verovnik et al. 2012: pp. 74). From authors' comments to the distribution map (cf. Verovnik et al. 2012: pp. 414) it is evident that this record originates from Hoffman \& Klos (1914) who mentioned the 


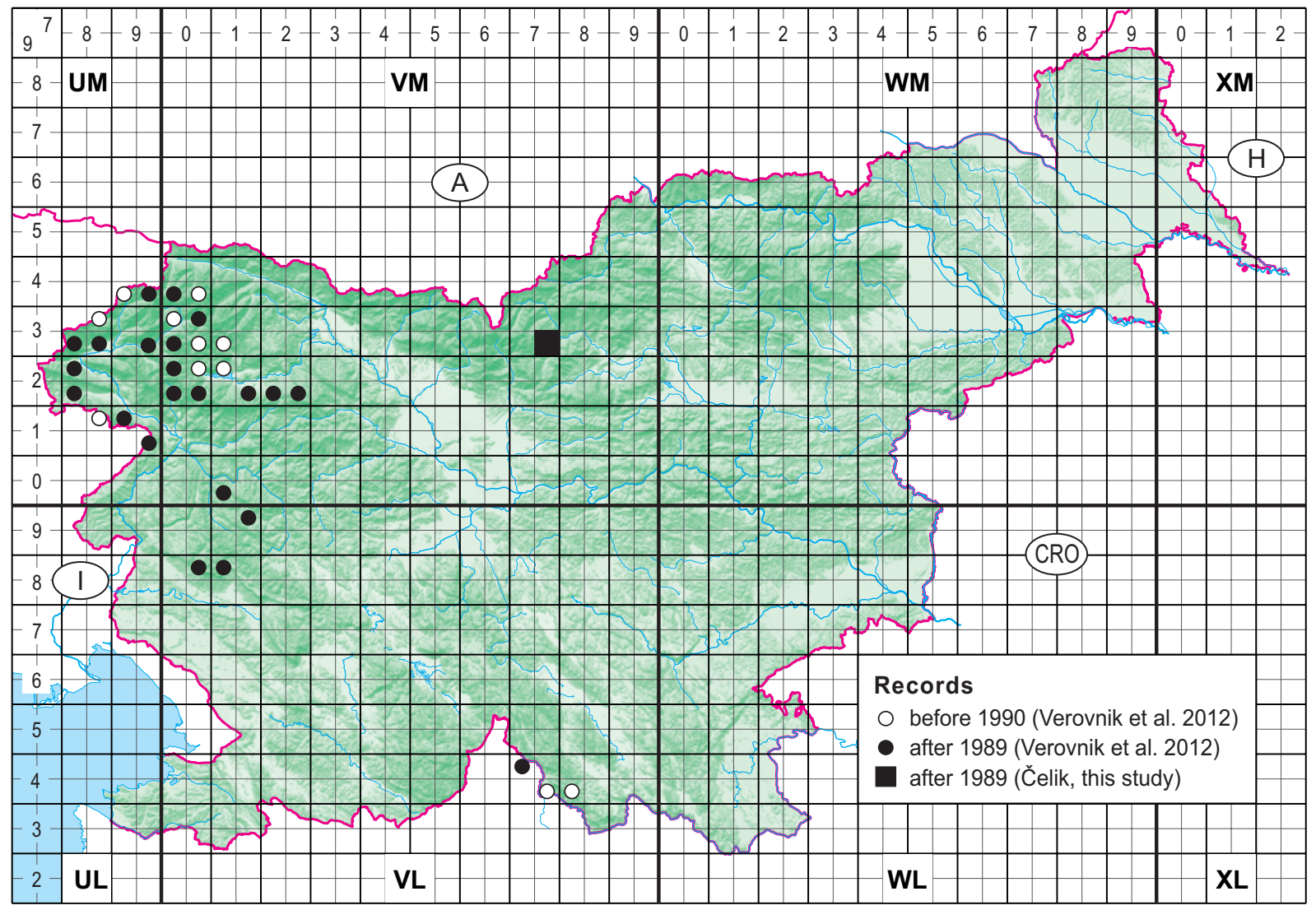

Figure 10: New records on the distribution of Erebia oeme (Hübner, 1804) (Čelik, this study) according to the Atlas of butterflies (Lepidoptera: Rhopalocera) of Slovenia (Verovnik et al. 2012: pp. 415).

Slika 10: Novi podatki o razširjenosti vrste Erebia oeme (Hübner, 1804) (Čelik, ta študija) glede na podatke v Atlasu dnevnih metuljev (Lepidoptera: Rhopalocera) Slovenije (Verovnik s sod. 2012: str. 415).

locality at Savinja river spring (Hoffman \& Klos 1914, pp. 260: "Am Rinka"). The distribution record from the second source related to historical data of species presence in the Kamniško-Savinjske Alps which the authors of the Atlas refer on (i.e. Sijarić 1991, pp. 317: Mt. Krvavec, 4.7.1978) is not presented on the species distribution map although its class of spatial accuracy corresponds to category 3 which is the smallest precision used in distribution maps (cf. Verovnik et al. 2012: pp. 74, but see also Verovnik et al. 2012: pp. 37).

\section{Erebia pandrose (Borkhausen, 1788)}

(I) - VM23/1: 17.7.2009, Julijske Alpe, Pokljuka, Pl. Klek, 1510 m, 420410/139143.

(II) - UM83/1: 18.7.2006 Slovenija, Primorska, Julijske Alpe, Bovec, Prevala, $2224 \mathrm{~m}$, 383292/136837; Vršiči pod Lopo, 2146 m, 383667/ 137165; VM73/4: 21.7.2009, Kamniško-Savinjske Alpe, Dleskovška planota, Dleskovec, $1850 \mathrm{~m}$, 476127/134010; $1880 \mathrm{~m}, 475603 / 134083 ; 1835 \mathrm{~m}$,
475535/134610; $1795 \mathrm{~m}, 476057 / 134883 ; 1670 \mathrm{~m}$, 476345/134925; 1715 m, 476193/134967; Smrekovec, $1590 \mathrm{~m}, 476702 / 135032$.

(III) - UM83/3: 18.7.2006, Julijske Alpe, Bovec, Črnelski Vršiči, $1740 \mathrm{~m}, 387130 / 136950$; $1780 \mathrm{~m}, 387060 / 137280 ; 2028 \mathrm{~m}, 386050 / 137430$; $2157 \mathrm{~m}, 386303 / 137855 ; 2115 \mathrm{~m}, 386560 / 137970$; 2320 m, 386162/137990; VM62/1: 15.7.2005, Kamniško-Savinjske Alpe, Ambrož pod Krvavcem, Veliki Zvoh, 1910 m, 464874/129340; 1953 m, 465167/129628; VM63/2: 16.7.2005, Kamniško-Savinjske Alpe, Grintovec, Spodnje jame, 1920 m, 465166/134016; Spodnji Dolci, 1850 m, 464340/134200; 1805 m, 464062/134294; 16.7.1995, Kamniško-Savinjske Alpe, Grintovec, Ovnov čer, 2126 m, 464752/134096.

(ii) - The presence of species in areas which in the Atlas are designated as "white spots": the eastern Julian Alps, the eastern part of Kamniško-Savinjske Alps. 
(iv) - New information on species occurrence in Kamniško-Savinjske Alps: species distribution map in the Atlas (Verovnik et al. 2012: pp. 417) shows that in that area there is only one $5 \times 5$ UTM square with species presence after year 1989, and five $5 \times 5$ UTM squares with records before year 1990. According to the authors comments (cf. Verovnik et al. 2012: pp. 416) the "species is widespread in the Kamniško-Savinjske Alps while in Karavanke Mountains is much more local" (ibid., pp. 416), although there are six $5 \times 5$ UTM squares with species presence in the Karavanke mountains after year 1989 , and two $5 \times 5$ UTM squares with records before year 1990. My records from Mt. Krvavec (VM62/1), Mt. Grintovec (VM63/2) and Dleskovška planota (VM73/4) show that species was observed in the Kamniško-Savinjske Alps in the last 10 years at more than one locality, but it still does not mean that the species is more widespread in the Kamniško-Savinjske Alps than in the Karavanke Mountains.

\section{Melanargia galathea (Linnaeus, 1758)}

(II) - UM90/4: 30.6.2006, Primorska, Banjšice, Breščakim 632 m, 398146/101314; VL23/2: 21.6.2002, Primorska, Rakitovec, Ubred, $660 \mathrm{~m}$, 421031/36003; VL99/3: 11.7.2003, Dolenjska, Polšnik, Golek, $690 \mathrm{~m}, 497957 / 100781 ; 680 \mathrm{~m}$, 498250/101025; VM10/2: 16.6.2006, Primorska, Dolenja Trebuša, Hotenja, 291 m, 412094/104768; 265 m, 411914/105006; VM32/1: 17.7.2005, Julijske Alpe, Jelovica, Spodnja Lipnica, $1001 \mathrm{~m}$, 433570/130640; VM72/3: 21.7.2006, Gorenjska, Krivčevo, Rakov rob, 1133 m, 475975/127144; VM90/3: 23.6.2011, Dolenjska, Spodnji Šklendrovec, 300 m, 500262/106812; WM22/1: 29.6.2005, Štajerska, Frankolovo, Rakova Steza, 330 m, 524113/130770.

(III) - VL16/2: 25.6.1997, Primorska, Sežana, Dol Leskovec, 300 m, 412212/62044; Dane pri Sežani, Koblak, 350 m, 414584/64790; VM94/2: 18.7.2005, Štajerska, Bele Vode, Senovršnik, $940 \mathrm{~m}, 495278 / 142158$.

\section{Minois dryas (Scopoli, 1763)}

(II) - UL93/3: 30.7.1996, Primorska, Puče, $232 \mathrm{~m}, 400285 / 37345$; UL99/3: 22.9.2006, 5.9.2006, 23.8.2006, Primorska, Banjšice, Visoko, $755 \mathrm{~m}, 399727 / 98814 ;$ 5.9.2006, 23.8.2006, Primorska, Banjšice, Visoko, 713 m, 399375/98918; 22.9.2006, 5.9.2006, 23.8.2006, Primorska, Banjšice, Kuk, 760 m, 400311/99855; Sleme, 769 m, 399542/100466; VL16/3: 2.9.2005, Primorska,
Kras, Griže, 510 m, 418829/67950; 1.8.2005, Primorska, Kras, Griže, 478 m, 418688/68001; VL 68/4: 14.7.2012, Dolenjska, Visoko, Vrh, 613 m, 467930/82100.

(ii) - The presence of species in areas which in the Atlas are designated as "white spots": Banjška planota, central part of Slovenia.

\section{Hipparchia fagi (Scopoli, 1763)}

(I) - WM23/2: 29.6.2005, Štajerska, Lipa pri Frankolovem, Selce, 633 m, 523152/134338.

\section{Hipparchia semele (Linnaeus, 1758)}

(I) - VL37/3: 4.8.1993, Notranjska, Planinsko polje, Sv. Jedert, 445 m, 440300/80195.

(II) - VL23/2: 21.6.2002, Primorska, Rakitovec, Ubred, $660 \mathrm{~m}, 421031 / 36003$; VL45/4: 9.7. 1994, Notranjska, Koritnice, Suha reber, $671 \mathrm{~m}$, 445539/51767; 19.8.1993, Notranjska, Koritnice, Jelenji Vršič, 688 m, 445539/52012; VL47/1: 4.8. 1993, Notranjska, Planinsko polje, Sv. Jedert, $445 \mathrm{~m}, 440390 / 80165$.

(ii) - The presence of species in areas which in the Atlas are designated as "white spots": the central part of Notranjska region.

\section{Arethusana arethusa (Denis \& Schiffermüller, 1775)}

(II) - UL99/3: 5.9.2006, 23.8.2006, Primorska, Banjšice Visoko, 755 m, 399727/98814; 22.9.2006, Primorska, Banjšice, Sleme, 769 m, 399542/100466.

(ii) - The presence of species in areas which in the Atlas are designated as "white spots": Banjška planota. It is the northernmost site of the species distribution in Slovenia after year 1989 (cf. Verovnik et al. 2012: pp. 431).

\section{Brintesia circe (Fabricius, 1775)}

(I) - VM11/4: 17.7.2006, Primorska, Baška grapa, Hudajužna, 424 m, 416263/114968; Obloke, 558 m, 416154/115482; WM03/1: 27.7.1987, Štajerska, Šoštanj, 359 m, 505112/137300; WM23/2: 29.6.2005, Štajerska, Straža pri Novi Cerkvi, Zg. Razdelj, 410 m, 522771/131829; Lipa pri Frankolovem, Selce, 633 m, 523152/134338; Zgornje Selce, $659 \mathrm{~m}, 522566 / 134766$.

(II) - UM90/4: 30.6.2006, Primorska, Banjšice, Breščaki, 632 m, 398146/101314; VL06/3: 5.7.2002, Primorska, Kras, Dutovlje, $275 \mathrm{~m}$, 408528/69110; Pliskovica, 290 m, 406303/70780; 
VM94/4: 18.7.2005, Štajerska, Zavodnje, Vovke, $590 \mathrm{~m}, 499460 / 141500$; Slopšnek, $750 \mathrm{~m}$, 499272/142756; Bele Vode, Tolsti vrh, $1087 \mathrm{~m}$, 496380/143168; Šentvid pri Zavodnju, $934 \mathrm{~m}$, 498634/143192; WM22/1: 29.6.2005, Štajerska, Frankolovo, Rakova Steza, 330 m, 524113/130770.

(III) - VL47/4: 27.7.1994, Notranjska, Cerknica, Kamna Gorica, 620 m, 450339/73257.

(ii) - The presence of species in areas which in the Atlas are designated as "white spots": Cerkljansko hills, Velenjsko and Konjiško hills.

\section{ACKNOWLEDGEMENTS}

I am grateful to M. Kuntner (Head of Jovan Hadži Institute of Biology) for valuable comments on the manuscript, and to I. Sajko for making the maps. Research facilities were provided by the Jovan Hadži Institute of Biology of the Scientific Research Centre of the Slovene Academy of Sciences and Arts.

\section{REFERENCES}

Čelik, T. 2011: Uvodnik. Svet ptic 17/3: 3.

Čelik, T. 2012: Munčev okarček Coenonympha tullia (Müller, 1764) (Lepidoptera: Nymphalidae) v Sloveniji ni izumrl. Acta entomologica slovenica 20: 45-58.

Čelik, T., Rebeušek, F., 1996: Atlas ogroženih vrst dnevnih metuljev Slovenije. Slovensko entomološko društvo Štefana Michielija, Ljubljana, $100 \mathrm{pp}$.
Čelik, T., Verovnik, R., Rebeušek, F., Gomboc, S., Lasan, M., 2004: Strokovna izhodišča za vzpostavljanje omrežja NATURA 2000: Metulji (Lepidoptera). Končno poročilo. Biološki inštitut Jovana Hadžija ZRC SAZU, Ljubljana, $297 \mathrm{pp}$.

Čelik, T., Verovnik, R., Gomboc, S., Lasan, M., 2005: Natura 2000 v Sloveniji - Metulji (Lepidoptera). Založba ZRC, ZRC SAZU, Ljubljana, $288 \mathrm{pp}$.

Hoffmann, F., Klos, R., 1914: Die Schmetterlinge Steiermarks. Sonder-Abdruck aus den Mitteilungen des Naturwissenschaftlichen Vereins für Steiermark, Jahrgang 1913, Band 50: 184-323.

Perko, D., Kladnik, D., 1998: Nova regionalizacija Slovenije. V: Perko, D., Adamič, M.O. (ur.): Slovenija - pokrajine in ljudje. Založba Mladinska knjiga, Ljubljana, pp. 26-31.

Sijarić, R., 1991: Katalog naučne zbirke Lepidoptera (Insecta) donatora Bore Mihljevića iz Sarajeva. Glasnik Zemaljskog muzeja Bosne i Hercegovine, Prirodne nauke, Nova serija 30: 169-360.

Van Swaay, C., Cuttelod, A., Collins, S., Maes, D., López Munguira, M., Šašić, M., Settele, J., Verovnik, R., Verstrael, T., Warren, M., Wiemers, M., Wynhof, I., 2010: European Red List of Butterfies. Publications Office of the European Union, Luxembourg, 47 pp.

Verovnik, R., Rebeušek, F., Jež, M., 2012: Atlas dnevnih metuljev (Lepidoptera: Rhopalocera) Slovenije, Atlas of butterflies (Lepidoptera: Rhopalocera) of Slovenia. Center za kartografijo favne in flore, Miklavž na Dravskem polju, $456 \mathrm{pp}$. 